TRANSACTIONS OF THE

AMERICAN MATHEMATICAL SOCIETY

Volume 366, Number 6, June 2014, Pages 2827-2888

S 0002-9947(2014)05770-X

Article electronically published on February 6, 2014

\title{
ON ASYMPTOTIC STABILITY OF MOVING GROUND STATES OF THE NONLINEAR SCHRÖDINGER EQUATION
}

\author{
SCIPIO CUCCAGNA
}

\begin{abstract}
We extend to the case of moving solitons, the result on asymptotic stability of ground states of the NLS obtained by the author in an earlier paper. For technical reasons we consider only smooth solutions. The proof is similar to the earlier paper. However now the flows required for the Darboux Theorem and the Birkhoff normal forms, instead of falling within the framework of standard theory of ODE's, are related to quasilinear hyperbolic symmetric systems. It is also not obvious that the Darboux Theorem can be applied, since we need to compare two symplectic forms in a neighborhood of the ground states not in $H^{1}\left(\mathbb{R}^{3}\right)$, but rather in the space $\Sigma$ where also the variance is bounded. But the NLS does not preserve small neighborhoods of the ground states in $\Sigma$.
\end{abstract}

\section{INTRODUCTION}

We consider the nonlinear Schrödinger equation (NLS)

$$
\mathrm{i} u_{t}=-\Delta u+\beta\left(|u|^{2}\right) u \quad, \quad u(0, x)=u_{0}(x),(t, x) \in \mathbb{R} \times \mathbb{R}^{3},
$$

assuming: $\beta\left(|u|^{2}\right) u$ is "short range" and smooth; (1.1) has a smooth family of ground states.

In Cu1 we proved that, if we break the translation invariance of the equations by either taking solutions with $u_{0}(-x) \equiv u_{0}(x)$ or by introducing some spacial inhomogeneity in the equation, for instance by adding a term $V(x) u$, the ground states are asymptotically stable, under what looks like a generic hypothesis. More precisely, we assumed the sufficient conditions for orbital stability by M. Weinstein W1. We assumed some spectral hypotheses on the linearizations (absence of embedded eigenvalues; this probably always holds true under Weinstein's hypotheses) and a number of other hypotheses which hold generically (nondegeneracy of the thresholds of the continuous spectrum; some mild nonresonance conditions on the eigenvalues) and which are stated in Subsection 1.1. We then proved a form of the Fermi golden rule (FGR). Specifically, we proved that certain coefficients of the system are square powers. This implies that they are nonnegative. We then assumed that these coefficients are in fact positive, which is probably true generically, and used this to prove asymptotic stability of the ground states. A result similar to Cu1, with some restrictions, is proved for the Dirac equation in [BuC].

In this paper we extend the proof of [Cu1 to equations like (1.1) without requiring any symmetry for the initial data $u_{0}$. Hence we prove that a solution $u(t)$ of (1.1) starting sufficiently close to ground states, is asymptotic as $t \nearrow+\infty$ of the

Received by the editors July 25, 2011 and, in revised form, October 22, 2011 and December 5, 2011.

2010 Mathematics Subject Classification. Primary 35Q55.

(C)2014 American Mathematical Society 
form $e^{i \theta(t)+\frac{i}{2} v_{+} \cdot x} \phi_{\omega_{+}}(x-D(t))+e^{i t \Delta} h_{+}$, for $\omega_{+}$and $v_{+}$fixed, for $\theta \in C^{1}(\mathbb{R}, \mathbb{R})$, $D \in C^{1}\left(\mathbb{R}, \mathbb{R}^{3}\right)$ and for $h_{+} \in H^{1}\left(\mathbb{R}^{3}\right)$ a small energy function. For technical reasons we need a certain known a priori regularity and decay of $u_{0}$, although in the estimates we use only the norm $\left\|u_{0}\right\|_{H^{1}}$.

The problem of stability of ground states of the NLS has a long history, partially reviewed in Cu1. Orbital stability was well understood in the 1980s (see CL, S, W1, GSS1, GSS2 ], and there is a long literature. For asymptotic stability we highlight [SW1, SW2, BP1, BP2, GS]; for more references see Cu1.

One of the crucial difficulties in asymptotic stability is in showing that the discrete modes appearing naturally in the problem, which left on their own would oscillate, are dumped by the nonlinear interaction with the continuous modes. By conservation of energy, this happens by passage of energy from the discrete to the continuous modes. The proof requires nonlinear versions of the FGR; see [Si]. In our setup, versions of the FGR of ever growing generality were proved in special cases in [BP2, SW3, TY1, TY2, TY3, Ts, BS, Cu2, GS, CM, GW]. They included the search of appropriate coordinates through Poincaré Dulac normal forms. For related linear problems, see $[\mathrm{KW}$ and the references therein. $\mathrm{Cu} 3$ seems to be the first reference to have noticed the relevance of the Hamiltonian structure of the NLS. The intuition in $\mathrm{Cu} 3$, was implemented in the series $\mathrm{BC}, \mathrm{Cu} 1, \mathrm{Cu} 4, \mathrm{BuC}$.

The FGR consists in proving that certain coefficients are square powers, and so are generally positive. The coefficients will typically have the structure $A \cdot \bar{B}$, with $A$ and $B$ coefficients of the system in appropriate coordinate systems. The square power structure will follow from $B=A$. Proving such identities among the coefficients in the NLS is certainly easier if we exploit the Hamiltonian structure. We search an appropriate system of coordinates through the method of Birkhoff normal forms. In the cases considered in [BC, $\mathrm{Bu} 4$ this is easier because the natural coordinates which appear linearizing the system around the 0 solution are canonical coordinates. So one can start the Birkhoff normal forms from the initial system of coordinates.

In analysis of the stability of solitons, the natural coordinates of the linearization are not canonical. Before starting the method of normal forms, one has to find canonical coordinates through an implementation of the Darboux Theorem. This has to be done in the right nonabstract way, in order not to lose the property that the NLS is a semilinear system. This process is done in [Cu1, BuC. However these papers, as well as most of the papers quoted so far, avoid the case of moving solitons. Special cases, without discrete modes, were treated in BP1,Cu5]. For multisolitons with weak interaction and no discrete modes, see [P] RSS.

Moving solitons present three special difficulties. First of all, they yield terms in the equation of the continuous modes which are nonlinear but which are difficult to see as perturbations of the linear equation. It is not obvious how to eliminate them through an integrating factor. Fortunately work by Beceanu, such as $\mathrm{Be}$, has solved this problem. Early solutions in particular cases are in [BP1,BP2] (see BS, $\mathrm{Cu} 5$ for proofs).

The second difficulty involves the Darboux Theorem. The method followed in Cu1 becomes too complicated in the moving solitons setting. It is useful to use charge and linear momenta as coordinates. In the case of the charge, had this been done in [Cu1, it would have simplified the proof there. One difficulty with the Darboux Theorem is the determination of the vectorfield $\mathcal{X}^{t}$ obtained as a dual of 
an appropriate 1 form, in the Moser version of the Darboux Theorem used here. In $\mathrm{Cu} 1$ the existence of such $\mathcal{X}^{t}$ and some of its properties are rather elementary. In this paper, we are comparing two symplectic forms which are not both defined in $H^{1}\left(\mathbb{R}^{3}\right)$. Rather, they are symplectic forms in the smaller space $\Sigma_{1}$ formed by functions of bounded $H^{1}$ norm and bounded variance; see (1.8) and Section 7 . The proof of the existence of $\mathcal{X}^{t}$ would be easy if we could assume that the variance of the solutions of the NLS, assuming it is small at time $t=0$, remained small for all times. But this is not the case, so the discussion is rather complicated.

The third difficulty present here and not in [Cu1 is that the vector fields whose flows are used to change coordinates in the implementation of the Darboux Theorem and of the method of Birkhoff normal forms do not fall as in [Cu1] within the framework of smooth vectorfields in Banach spaces. Here instead we have to deal with quasilinear hyperbolic symmetric systems. So wellposedness and regularity of the flows, which in Cu1 are elementary, are more delicate here. Particular attention requires the issue of regularity of the flows with respect to the initial data. Fortunately our systems have a quite simple structure. In a rather standard way, our flows are obtained as limits of flows of systems with viscosity, which fall within the classical framework of ODE's. In the limit we lose some regularity. It is at this juncture that we use the qualitative information on regularity and decay of the initial datum $u_{0}$. The more we iterate, the more we lose regularity. Fortunately we have as much regularity and decay of $u_{0}$ and of the ground states as we want, to start with.

1.1. Statement of the main result. We will assume the following hypotheses:

(H1) $\beta(0)=0, \beta \in C^{\infty}(\mathbb{R}, \mathbb{R})$.

(H2) There exists a $p \in(1,5)$ such that for every $k \geq 0$ there is a fixed $C_{k}$ with

$$
\left|\frac{d^{k}}{d v^{k}} \beta\left(v^{2}\right)\right| \leq C_{k}|v|^{p-k-1} \quad \text { if }|v| \geq 1 .
$$

(H3) There exists an open interval $\mathcal{O}$ such that

$$
\Delta u-\omega u+\beta\left(|u|^{2}\right) u=0 \quad \text { for } x \in \mathbb{R}^{3}
$$

admits a $C^{1}$-family of ground states $\phi_{\omega}(x)$ for $\omega \in \mathcal{O}$.

$$
\frac{d}{d \omega}\left\|\phi_{\omega}\right\|_{L^{2}\left(\mathbb{R}^{3}\right)}^{2}>0 \quad \text { for } \omega \in \mathcal{O} .
$$

(H5) Let $L_{+}=-\Delta+\omega-\beta\left(\phi_{\omega}^{2}\right)-2 \beta^{\prime}\left(\phi_{\omega}^{2}\right) \phi_{\omega}^{2}$ be the operator whose domain is $H^{2}\left(\mathbb{R}^{3}\right)$. Then we assume that $L_{+}$has exactly one negative eigenvalue and does not have kernel when restricted to $H_{r}^{1}\left(\mathbb{R}^{3}\right)$, the subspace of $H^{1}\left(\mathbb{R}^{3}\right)$ formed by functions with radial symmetry.

(H6) Let $\mathcal{H}_{\omega}$ be the linearized operator around $\phi_{\omega}$ (see Section 2 for the precise definition). $\mathcal{H}_{\omega}$ has $m$ positive eigenvalues $\lambda_{1}(\omega) \leq \lambda_{2}(\omega) \leq \ldots \leq \lambda_{m}(\omega)$ with $0<N_{j} \lambda_{j}(\omega)<\omega<\left(N_{j}+1\right) \lambda_{j}(\omega)$ with $N_{j} \geq 1$. We set $N=N_{1}$. Here we are repeating each eigenvalue a number of times equal to its multiplicity. We assume the multiplicity constant in $\omega$.

(H7) There is no multiindex $\mu \in \mathbb{Z}^{m}$ with $|\mu|:=\left|\mu_{1}\right|+\ldots+\left|\mu_{k}\right| \leq 2 N_{1}+3$ such that $\mu \cdot \lambda=m$. 
(H8) If $\lambda_{j_{1}}<\ldots<\lambda_{j_{k}}$ are $k$ distinct $\lambda$ 's, and $\mu \in \mathbb{Z}^{k}$ satisfies $|\mu| \leq 2 N_{1}+3$, then we have

$$
\mu_{1} \lambda_{j_{1}}+\cdots+\mu_{k} \lambda_{j_{k}}=0 \Longleftrightarrow \mu=0 .
$$

(H9) $\mathcal{H}_{\omega}$ has no other eigenvalues except for 0 and the $\pm \lambda_{j}(\omega)$. The points $\pm \omega$ are not resonances.

(H10) The Fermi golden rule of hypothesis (H10) in Subsection 13.2, see also (13.2), holds.

(H11) We assume that $u_{0}$ is a Schwartz function.

Recall that from the $\phi_{\omega}$ one can derive solitons $e^{\frac{i}{2} v \cdot x-\frac{i}{4}|v|^{2} t+i t \omega+i \gamma} \phi_{\omega}(x-v t-D)$. Solutions of (1.1) starting close to a ground state, for some time can be written as

$$
\begin{aligned}
& u(t, x)=\tau_{D(t)} e^{i \Theta(t, x)}\left(\phi_{\omega(t)}(x)+r(t, x)\right), \\
& \Theta(t, x)=\frac{1}{2} v(t) \cdot x+\vartheta(t)
\end{aligned}
$$

with $\tau_{D} f(x):=f(x-D)$.

Theorem 1.1. Let $\omega_{1} \in \mathcal{O}, v_{1} \in \mathbb{R}$ and $\phi_{\omega_{1}}(x)$ be a ground state of (1.1). Let $u(t, x)$ be a solution to (1.1). Assume (H1)-(H10). Then, there exist an $\epsilon_{0}>0$ and $a C>0$ such that if $\varepsilon:=\inf _{\gamma \in \mathbb{R}, y \in \mathbb{R}^{3}}\left\|u_{0}-e^{\mathrm{i} \gamma} e^{\frac{\mathrm{i}}{2} v_{1} \cdot x} \phi_{\omega_{1}}(\cdot-y)\right\|_{H^{1}}<\epsilon_{0}$, there exist $\omega_{ \pm} \in \mathcal{O}, v_{ \pm} \in \mathbb{R}^{3}, \theta \in C^{1}(\mathbb{R} ; \mathbb{R}), y \in C^{1}\left(\mathbb{R} ; \mathbb{R}^{3}\right)$ and $h_{ \pm} \in H^{1}$ with $\left\|h_{ \pm}\right\|_{H^{1}}+\left|\omega_{ \pm}-\omega_{1}\right|+\left|v_{ \pm}-v_{1}\right| \leq C \varepsilon$ such that

$$
\lim _{t \rightarrow \pm \infty}\left\|u(t, \cdot)-e^{\mathrm{i} \theta(t)+\frac{\mathrm{i}}{2} v_{ \pm} \cdot x} \tau_{y(t)} \phi_{\omega_{ \pm}}-e^{\mathrm{i} t \Delta} h_{ \pm}\right\|_{H^{1}}=0 .
$$

In the notation of (1.4), we have $\tau_{D(t)} e^{i \Theta(t, x)} r(t, x)=A(t, x)+\widetilde{r}(t, x)$ such that $A(t, \cdot) \in \mathcal{S}\left(\mathbb{R}^{3}, \mathbb{C}\right),|A(t, x)| \leq C(t)$ with $\lim _{|t| \rightarrow \infty} C(t)=0$ and such that for any pair $(p, q)$ which is admissible, by which we mean that

$$
2 / p+3 / q=3 / 2, \quad 6 \geq q \geq 2, \quad p \geq 2,
$$

we have

$$
\|\widetilde{r}\|_{L_{t}^{p}\left(\mathbb{R}, W_{x}^{1, q}\right)} \leq C\left\|u_{0}\right\|_{H^{1}} .
$$

Remark 1.2. In the proof we use only bounds on the $H^{1}$ norm of $u_{0}$. Nonetheless we use in a qualitative fashion the fact that $u_{0}$ is quite regular and rapidly decaying. For simplicity we restrict our attention to the case when $u_{0}$ is a Schwartz function.

Remark 1.3. In the proof we show that we can take $\theta=\vartheta$ and $y=D$, with $(\vartheta, D)$ the functions in Lemma 2.1. In Lemma 13.9 we show $\dot{D}=v+o(1)$ with $v$ as in Lemma 2.1 and $\lim _{t \rightarrow \infty} o(1)=0$. Similarly, $\dot{\vartheta}=\omega+\frac{v^{2}}{4}+o(1)$.

Remark 1.4. Notice that in (H6) we exclude eigenvalues of $\mathcal{H}_{\omega}$ in $(\omega, \infty)$ mainly because we think they do not exist under (H1)-(H5). Notice that in [CPV] smoothing estimates for $\mathcal{H}_{\omega}$ are also proved in the presence of eigenvalues in $(\omega, \infty)$, so that the theory here and in $\mathrm{Cu} 1$ ] could also be developed in that situation.

Remark 1.5. By elementary arguments, Theorem 1.1 is a consequence of the special case where the linear momenta are equal to 0 ; see (2.3). So we will focus only on this case. 
We briefly describe the proof, which is similar in spirit to [Cu1, but departs from [Cu1] in important ways. First of all, we need to choose a system of coordinates around the ground states. There is a natural choice related to the notion of modulation and to the spectral decomposition of the linearization. Only in a second moment do we use charge and linear moment as coordinates. Since these are invariants of motion, we then consider a reduction of coordinates, in an elementary fashion. We also move to canonical coordinates through an implementation of the Darboux Theorem. We then start the Birkhoff normal form argument, that is, we consider other canonical coordinates where the system looks increasingly more treatable. Finally, after a finite number of them, we settle with coordinates where it is possible to prove the Fermi golden rule. Then, if (H10) is true, we conclude simultaneously that the continuous modes disperse and that the energy of the discrete modes leaks away through nonlinear interaction with the continuous modes. The most delicate and novel feature of this paper with respect to [Cu1 consists in the analysis of the flows $\phi^{t}$ used for the Darboux Theorem and the Birkhoff normal forms. In particular, since we are outside the realm of ODE's, it is less obvious to conclude that for fixed $t$ the flow $\phi^{t}$ is a differentiable map. This is where (H11) is helpful. As for Birkhoff normal forms, we also add some more material useful to understand the homological equations, which are nonlinear, and which should help to understand the analogous discussion in Cu1, which is very succinct.

We end the introduction with some notation. Given two functions $f, g: \mathbb{R}^{3} \rightarrow \mathbb{C}$ we set $\langle f \mid g\rangle=\int_{\mathbb{R}^{3}} f(x) g(x) d x$ (with no complex conjugation). Given a matrix $A$, we denote by $A^{T}$, its transpose. Given two vectors $A$ and $B$, we denote by $A^{T} B=\sum_{j} A_{j} B_{j}$ their inner product. Sometimes we omit the summation symbol, and we use the convention of sum over repeated indexes. For any $k, s \in \mathbb{R}$ and any Banach space $K$, we set

$$
H^{k, s}\left(\mathbb{R}^{3}, K\right)=\left\{f: \mathbb{R}^{3} \rightarrow K \text { s.t. }\|f\|_{H^{s, k}}:=\left\|\langle x\rangle^{s}\right\|(-\Delta+1)^{k} f\left\|_{K}\right\|_{L^{2}}<\infty\right\} .
$$

In particular we set $L^{2, s}=H^{0, s}, L^{2}=L^{2,0}, H^{k}=H^{k, 0}$. Sometimes, to emphasize that these spaces refer to spatial variables, we will denote them by $W_{x}^{k, p}, L_{x}^{p}, H_{x}^{k}$, $H_{x}^{k, s}$ and $L_{x}^{2, s}$. For $I$ an interval and $Y_{x}$ any of these spaces, we will consider Banach spaces $L_{t}^{p}\left(I, Y_{x}\right)$ with mixed norm $\|f\|_{L_{t}^{p}\left(I, Y_{x}\right)}:=\|\| f\left\|_{Y_{x}}\right\|_{L_{t}^{p}(I)}$. In the course of the proof we will consider a fixed pair of spaces $H^{K, S}$ and $H^{-K,-S}$, for positive and large $K$ and $S$.

We set $\left(\mathrm{i} \partial_{x}+\mathrm{i} x\right)^{\alpha}:=\prod_{a=1}^{3}\left(\mathrm{i} \partial_{a}+\mathrm{i} x_{a}\right)^{\alpha_{a}}$ for any multiindex $\alpha$. For any natural number $n \geq 1$ we consider the space $\Sigma_{n}$ defined by

$$
\|U\|_{\Sigma_{n}}^{2}:=\sum_{|\alpha| \leq n}\left\|\left(\mathrm{i} \partial_{x}+\mathrm{i} x\right)^{\alpha} U\right\|_{L^{2}}^{2}<\infty
$$

Given an operator $A$, we will denote by $R_{A}(z)=(A-z)^{-1}$ its resolvent. We set $\mathbb{N}_{0}=\mathbb{N} \cup\{0\}$. We will consider multiindexes $\mu \in \mathbb{N}_{0}^{m}$. For $\mu \in \mathbb{Z}^{m}$ with $\mu=\left(\mu_{1}, \ldots, \mu_{m}\right)$ we set $|\mu|=\sum_{j=1}^{m}\left|\mu_{j}\right|$. For $X$ and $Y$ two Banach spaces, we will denote by $B(X, Y)$ the Banach space of bounded linear operators from $X$ to $Y$ and by $B^{\ell}(X, Y)=B\left(\prod_{j=1}^{\ell} X, Y\right)$. Given a differential form $\alpha$, we denote by $d \alpha$ its exterior differential. 


\section{LiNEARIZATION AND SETUP}

Let $U={ }^{t}(u, \bar{u})$. We consider the energy

$$
\begin{aligned}
& E(U)=E_{K}(U)+E_{P}(U), \\
& E_{K}(U):=\int_{\mathbb{R}^{3}} \nabla u \cdot \nabla \bar{u} d x, \quad E_{P}(U):=\int_{\mathbb{R}^{3}} B(u \bar{u}) d x
\end{aligned}
$$

with $B(0)=0$ and $\partial_{\bar{u}} B\left(|u|^{2}\right)=\beta\left(|u|^{2}\right) u$. We will consider the matrices

$$
\sigma_{1}=\left(\begin{array}{ll}
0 & 1 \\
1 & 0
\end{array}\right), \sigma_{2}=\left(\begin{array}{cc}
0 & \mathrm{i} \\
-\mathrm{i} & 0
\end{array}\right), \sigma_{3}=\left(\begin{array}{cc}
1 & 0 \\
0 & -1
\end{array}\right) .
$$

For $U \in H^{1}\left(\mathbb{R}^{3}, \mathbb{C}\right) \times H^{1}\left(\mathbb{R}^{3}, \mathbb{C}\right)$ we have the following charge and momenta, which yield invariants of motion of (1.1):

$$
\begin{aligned}
& Q(U)=\int_{\mathbb{R}^{3}} u \bar{u} d x=\frac{1}{2}\left\langle U \mid \sigma_{1} U\right\rangle, \\
& \Pi_{a}(U)=\operatorname{Im} \int_{\mathbb{R}^{3}} \bar{u}(x) u_{x_{a}}(x) d x=-\frac{\mathrm{i}}{2}\left\langle U \mid \sigma_{3} \sigma_{1} \frac{\partial}{\partial x_{a}} U\right\rangle .
\end{aligned}
$$

Sometimes we will denote $\Pi(U)=\left(\Pi_{1}(U), \Pi_{1}(U), \Pi_{3}(U)\right)$. We will focus only on solutions of the NLS (1.1) s.t. $\Pi(U)=0$.

The charge $Q$ and the momenta $\Pi_{a}$ are in $C^{\infty}\left(H^{1}\left(\mathbb{R}^{3}, \mathbb{C}\right) \times H^{1}\left(\mathbb{R}^{3}, \mathbb{C}\right), \mathbb{C}\right)$ while $E \in C^{1}\left(H^{1}\left(\mathbb{R}^{3}, \mathbb{C}\right) \times H^{1}\left(\mathbb{R}^{3}, \mathbb{C}\right), \mathbb{C}\right)$. If for any such functional $F$ we set $d F(X)=$ $\langle\nabla F, X\rangle$ for any $X \in \mathbb{C}^{2}$, with $d F$ the exterior differential and $\nabla F$ the gradient of $F$, then

$$
\nabla Q(U)=\sigma_{1} U, \quad \nabla \Pi_{a}(U)=-\mathrm{i} \sigma_{3} \sigma_{1} \frac{\partial}{\partial x_{a}} U
$$

For later use we set

$$
\begin{aligned}
& \Phi_{\omega}=\left(\begin{array}{c}
\phi_{\omega} \\
\phi_{\omega}
\end{array}\right), q(\omega)=Q\left(\Phi_{\omega}\right), e(\omega)=E\left(\Phi_{\omega}\right), p_{a}(\omega)=\Pi_{a}\left(\Phi_{\omega}\right), \\
& d(\omega)=e(\omega)+\omega q(\omega) .
\end{aligned}
$$

Equation (1.1) can be written as

$$
\dot{\mathrm{i}} \dot{U}=\left(\begin{array}{cc}
0 & 1 \\
-1 & 0
\end{array}\right)\left(\begin{array}{c}
\partial_{u} E \\
\partial_{\bar{u}} E
\end{array}\right)=\sigma_{3} \sigma_{1} \nabla E(U) .
$$

We now introduce the linearization

$$
\begin{aligned}
& \mathcal{H}_{\omega}:=\sigma_{3} \sigma_{1}\left(\nabla^{2} E\left(\Phi_{\omega}\right)+\omega \nabla^{2} Q\left(\Phi_{\omega}\right)\right)=\sigma_{3}(-\Delta+\omega)+V_{\omega}, \\
& \text { where } V_{\omega}:=\sigma_{3}\left[\beta\left(\phi_{\omega}^{2}\right)+\beta^{\prime}\left(\phi_{\omega}^{2}\right) \phi_{\omega}^{2}\right]+\mathrm{i} \sigma_{2} \beta^{\prime}\left(\phi_{\omega}^{2}\right) \phi_{\omega}^{2} .
\end{aligned}
$$

The essential spectrum of $\mathcal{H}_{\omega}$ is $(-\infty,-\omega] \cup[\omega,+\infty)$. It is well known, WW2, that by (H4)-(H5) 0 is an isolated eigenvalue of $\mathcal{H}_{\omega}$ with $\operatorname{dim} N_{g}\left(\mathcal{H}_{\omega}\right)=8$ and

$$
\begin{aligned}
& \mathcal{H}_{\omega} \sigma_{3} \Phi_{\omega}=0=\mathcal{H}_{\omega} \partial_{x_{j}} \Phi_{\omega}, \\
& \mathcal{H}_{\omega} \partial_{\omega} \Phi_{\omega}=-\Phi_{\omega}, \quad \mathcal{H}_{\omega} x_{a} \sigma_{3} \Phi_{\omega}=-\partial_{x_{a}} \Phi_{\omega} .
\end{aligned}
$$

Since $\mathcal{H}_{\omega}^{*}=\sigma_{3} \mathcal{H}_{\omega} \sigma_{3}$, we have $N_{g}\left(\mathcal{H}_{\omega}^{*}\right)=\operatorname{span}\left\{\Phi, \sigma_{3} \partial_{\omega} \Phi, \sigma_{3} \partial_{x_{a}} \Phi, x_{a} \Phi\right\}$. We consider eigenfunctions $\xi_{j}(\omega)$ with eigenvalue $\lambda_{j}(\omega)$ :

$$
\mathcal{H}_{\omega} \xi_{j}(\omega)=\lambda_{j}(\omega) \xi_{j}(\omega), \quad \mathcal{H}_{\omega} \sigma_{1} \xi_{j}(\omega)=-\lambda_{j}(\omega) \sigma_{1} \xi_{j}(\omega) .
$$


They can be normalized so that $\left\langle\sigma_{3} \mathcal{H}_{\omega} \xi_{j}(\omega), \bar{\xi}_{\ell}(\omega)\right\rangle=\delta_{j \ell}$. Furthermore, they can be chosen to be real, that is, with real entries, so $\xi_{j}=\bar{\xi}_{j}$ for all $j$; see Proposition 5.1 of $\mathrm{GW}$.

Both $\phi_{\omega}$ and $\xi_{j}(\omega, x)$ are smooth in $\omega \in \mathcal{O}$ and $x \in \mathbb{R}^{3}$ and satisfy

$$
\sup _{\omega \in \mathcal{K}, x \in \mathbb{R}^{3}} e^{a|x|}\left(\left|\partial_{x}^{\alpha} \phi_{\omega}(x)\right|+\sum_{j=1}^{m}\left|\partial_{x}^{\alpha} \xi_{j}(\omega, x)\right|\right)<\infty
$$

for every $a \in\left(0, \inf _{\omega \in \mathcal{K}} \sqrt{\omega-\lambda(\omega)}\right)$ and every compact subset $\mathcal{K}$ of $\mathcal{O}$.

For $\omega \in \mathcal{O}$, we have the $\mathcal{H}_{\omega}$-invariant Jordan block decomposition

$$
L^{2}\left(\mathbb{R}^{3}, \mathbb{C}^{2}\right)=N_{g}\left(\mathcal{H}_{\omega}\right) \oplus\left(\bigoplus_{ \pm} \bigoplus_{j=1}^{m} \operatorname{ker}\left(\mathcal{H}_{\omega} \mp \lambda_{j}(\omega)\right)\right) \oplus L_{c}^{2}\left(\mathcal{H}_{\omega}\right),
$$

$L_{c}^{2}\left(\mathcal{H}_{\omega}\right):=\left\{N_{g}\left(\mathcal{H}_{\omega}^{*}\right) \oplus\left(\bigoplus_{\lambda \in \sigma_{d} \backslash\{0\}} \operatorname{ker}\left(\mathcal{H}_{\omega}^{*}-\lambda(\omega)\right)\right)\right\}^{\perp}$ with $\sigma_{d}=\sigma_{d}\left(\mathcal{H}_{\omega}\right)$. We also set $L_{d}^{2}\left(\mathcal{H}_{\omega}\right):=N_{g}\left(\mathcal{H}_{\omega}\right) \oplus\left(\bigoplus_{\lambda \in \sigma_{d} \backslash\{0\}} \operatorname{ker}\left(\mathcal{H}_{\omega}-\lambda(\omega)\right)\right)$. By $P_{c}\left(\mathcal{H}_{\omega}\right)$ (resp. $P_{d}\left(\mathcal{H}_{\omega}\right)$ ), or simply by $P_{c}(\omega)$ (resp. $P_{d}(\omega)$ ), we denote the projection on $L_{c}^{2}\left(\mathcal{H}_{\omega}\right)$ $\left(\right.$ resp. $\left.L_{d}^{2}\left(\mathcal{H}_{\omega}\right)\right)$ associated to the above direct sum. The spectral decomposition of a vector $X$ with respect to (2.9) is

$$
\begin{aligned}
& X=\left[P_{N_{g}\left(\mathcal{H}_{\omega}\right)}+\sum_{j=1}^{m}\left(P_{\operatorname{ker}\left(\mathcal{H}_{\omega}-\lambda_{j}\right)}+P_{\operatorname{ker}\left(\mathcal{H}_{\omega}+\lambda_{j}\right)}\right)+P_{c}\left(\mathcal{H}_{\omega}\right)\right] X \\
& =\frac{\left\langle X \mid \sigma_{3} \partial_{\omega} \Phi\right\rangle}{q^{\prime}(\omega)} \sigma_{3} \Phi+\frac{\langle X \mid \Phi\rangle}{q^{\prime}(\omega)} \partial_{\omega} \Phi+\sum_{a=1}^{3} \frac{\left\langle X \mid x_{a} \Phi\right\rangle}{q(\omega)} \partial_{x_{a}} \Phi-\sum_{a=1}^{3} \frac{\left\langle X \mid \sigma_{3} \partial_{x_{a}} \Phi\right\rangle}{q(\omega)} \sigma_{3} x_{a} \Phi \\
& +\sum_{j=1}^{m}\left\langle X \mid \sigma_{3} \xi_{j}\right\rangle \xi_{j}+\sum_{j=1}^{m}\left\langle X \mid \sigma_{1} \sigma_{3} \xi_{j}\right\rangle \sigma_{1} \xi_{j}+P_{c}\left(\mathcal{H}_{\omega}\right) X
\end{aligned}
$$

The following lemma is well known.

Lemma 2.1. Fix $U_{o}=e^{\mathrm{i} \sigma_{3}\left(\frac{v_{o} \cdot\left(x-D_{o}\right)}{2}+\vartheta_{o}\right)} \Phi_{\omega_{o}}\left(x-D_{o}\right)$. Then $\exists$ a neighborhood $\mathcal{U}^{1,0}$ of $U_{o}$ in $H^{1}$ and functions $\omega \in C^{\infty}\left(\mathcal{U}^{1,0}, \mathcal{O}\right), \vartheta \in C^{\infty}\left(\mathcal{U}^{1,0}, \mathbb{R}\right)$ and $D, v \in$ $C^{\infty}\left(\mathcal{U}^{1,0}, \mathbb{R}^{3}\right)$, s.t. in $U_{o}$ their value is $\left(\omega_{o}, \vartheta_{o}, D_{o}, v_{o}\right)$ and s.t. $\forall U \in \mathcal{U}^{1,0}$

$$
U(x)=e^{\mathrm{i} \sigma_{3}\left(\frac{v \cdot(x-D)}{2}+\vartheta\right)}\left(\Phi_{\omega}(x-D)+R(x-D)\right) \text { and } R \in N_{g}^{\perp}\left(\mathcal{H}_{\omega}^{*}\right) .
$$

Notice that, once the functions are given, we have

$$
R(x)=e^{-\mathrm{i} \sigma_{3}\left(\frac{v \cdot x}{2}+\vartheta\right)} U(x+D)-\Phi_{\omega}(x)
$$

with the rhs just continuous in $U$. We can further decompose $R$ using (2.9) as

$$
R(x)=\sum_{j=1}^{m} z_{j} \xi_{j}(\omega, x)+\sum_{j=1}^{m} \bar{z}_{j} \sigma_{1} \xi_{j}(\omega, x)+P_{c}\left(\mathcal{H}_{\omega}\right) f(x), \quad f \in L_{c}^{2}\left(\mathcal{H}_{\omega_{0}}\right),
$$

where we fixed $\omega_{0} \in \mathcal{O}$ such that $q\left(\omega_{0}\right)=\left\|u_{0}\right\|_{2}^{2}$. So we have

$$
\begin{aligned}
& U(x)=e^{\mathrm{i} \sigma_{3}\left(\frac{v \cdot(x-D)}{2}+\vartheta\right)}\left(\Phi_{\omega}(x-D)+z \cdot \xi(\omega, x-D)\right. \\
& \left.+\bar{z} \cdot \sigma_{1} \xi(\omega, x-D)+\left(P_{c}\left(\mathcal{H}_{\omega}\right) f\right)(x-D)\right) .
\end{aligned}
$$


(2.14) is a system of coordinates because for $\mathcal{O}$ sufficiently small the map $P_{c}\left(\mathcal{H}_{\omega}\right)$ is an isomorphism from $L_{c}^{2}\left(\mathcal{H}_{\omega_{0}}\right)$ to $L_{c}^{2}\left(\mathcal{H}_{\omega}\right)$. Notice that the maps $U \rightarrow z_{j}$ are smooth.

We set $z \cdot \xi=\sum_{j} z_{j} \xi_{j}$ and $\bar{z} \cdot \sigma_{1} \xi=\sum_{j} \bar{z}_{j} \sigma_{1} \xi_{j}$. In the sequel we set

$$
\partial_{\omega} R=\sum_{j=1}^{m} z_{j} \partial_{\omega} \xi_{j}(\omega)+\sum_{j=1}^{m} \bar{z}_{j} \sigma_{1} \partial_{\omega} \xi_{j}(\omega)+\partial_{\omega} P_{c}\left(\mathcal{H}_{\omega}\right) f .
$$

Sometimes we will denote $P_{c}(\omega)=P_{c}\left(\mathcal{H}_{\omega}\right)$.

We have the following formulas:

$$
\begin{aligned}
& \frac{\partial}{\partial \omega}=e^{\mathrm{i} \sigma_{3} \Theta} \partial_{\omega}(\Phi(x-D)+R(x-D)), \quad \frac{\partial}{\partial \vartheta}=\mathrm{i} \sigma_{3} U(x), \\
& \frac{\partial}{\partial D_{a}}=-\frac{\partial}{\partial x_{a}} U(x), \quad \frac{\partial}{\partial v_{a}}=\frac{\mathrm{i}}{2} \sigma_{3}\left(x_{a}-D_{a}\right) U(x) \text { for } a=1,2,3, \\
& \frac{\partial}{\partial z_{j}}=e^{\mathrm{i} \sigma_{3} \Theta} \xi_{j}(x-D), \quad \frac{\partial}{\partial \bar{z}_{j}}=e^{\mathrm{i} \sigma_{3} \Theta} \sigma_{1} \xi_{j}(x-D) \text { for } j=1, \ldots, m .
\end{aligned}
$$

Lemmas 2.2 2.4 are similar to analogous ones in [Cu1].

Lemma 2.2. There is a matrix $\mathbb{A}$ such that

$$
\left(\begin{array}{c}
\left\langle\tau_{D} e^{-\mathrm{i} \sigma_{3} \Theta} \Phi\right| \\
\left\langle\tau_{D} e^{-\mathrm{i} \sigma_{3} \Theta} \sigma_{3} \partial_{\omega} \Phi\right| \\
2\left\langle\tau_{D} e^{-\mathrm{i} \sigma_{3} \Theta} \sigma_{3} \nabla_{x} \Phi\right| \\
\left\langle\tau_{D} e^{-\mathrm{i} \sigma_{3} \Theta} x \Phi\right|
\end{array}\right)=\mathbb{A}\left(\begin{array}{c}
-q^{\prime}(\omega) d \omega \\
-\mathrm{i} q^{\prime}(\omega) d \vartheta \\
\mathrm{i} q(\omega) d v \\
-q(\omega) d D
\end{array}\right)
$$

We have $\mathbb{A}=1+\mathbb{A}_{1}(z, f, \omega, v)$ with $\mathbb{A}_{1}=(1+|v|) O\left(|z|+\|f\|_{H^{-K,-S}}\right)$ smooth in the arguments $z \in \mathbb{C}^{m}, f \in H^{-K,-S}$ and $(\omega, v)$, for any pair $(K, S)$.

Proof. Consider the functions of the variable $(U, \omega, \vartheta, v, D)$

$$
\begin{aligned}
& \mathcal{F}:=\left\langle e^{-\mathrm{i} \sigma_{3}\left(\frac{v \cdot(x-D)}{2}+\vartheta\right)} U(x)-\Phi_{\omega}(x-D) \mid \Phi_{\omega}(x-D)\right\rangle, \\
& \mathcal{G}:=\left\langle e^{-\mathrm{i} \sigma_{3}\left(\frac{v \cdot(x-D)}{2}+\vartheta\right)} U(x)-\Phi_{\omega}(x-D) \mid \sigma_{3} \partial_{\omega} \Phi_{\omega}(x-D)\right\rangle, \\
& \mathcal{B}_{a}:=\left\langle e^{-\mathrm{i} \sigma_{3}\left(\frac{v \cdot(x-D)}{2}+\vartheta\right)} U(x)-\Phi_{\omega}(x-D) \mid\left(x_{a}-D\right) \Phi_{\omega}(x-D)\right\rangle, \\
& \mathcal{D}_{a}:=\left\langle e^{-\mathrm{i} \sigma_{3}\left(\frac{v \cdot(x-D)}{2}+\vartheta\right)} U(x)-\Phi_{\omega}(x-D) \mid \sigma_{3} \partial_{a} \Phi_{\omega}(x-D)\right\rangle .
\end{aligned}
$$

Notice that differentiating in $U$ we obtain the vectors on the lhs of (2.17), which therefore span a vector bundle which has as sections the gradients $\nabla \omega, \nabla \vartheta, \nabla v_{a}$, $\nabla D_{a}$. For $R(x)$ defined by (2.12), we have the following partial derivatives:

$$
\begin{aligned}
& \mathcal{G}_{\omega}=\left\langle R \mid \sigma_{3} \partial_{\omega}^{2} \Phi\right\rangle, \quad \mathcal{G}_{\vartheta}=\mathrm{i}\left(q^{\prime}(\omega)+\left\langle R \mid \partial_{\omega} \Phi\right\rangle\right), \\
& \mathcal{G}_{D_{a}}=\frac{\mathrm{i} v_{a} q^{\prime}(\omega)}{2}+\left\langle\left(\partial_{a}+\frac{\mathrm{i}}{2} \sigma_{3} v_{a}\right) R \mid \sigma_{3} \partial_{\omega} \Phi\right\rangle, \quad \mathcal{G}_{v_{a}}=-\frac{\mathrm{i}}{2}\left\langle x_{a} R \mid \partial_{\omega} \Phi\right\rangle .
\end{aligned}
$$

Similar formulas are satisfied by the other functionals in (2.18). Substituting decomposition (2.13), we see that the functions in (2.19) satisfy the regularity required for $\mathbb{A}_{1}$. For the other functions in (2.18) it is straightforward to check that the same is true. This yields Lemma 2.2 by an elementary argument. 
Lemma 2.3. For $\mathcal{U}^{1,0}$ in Lemma 2.1 sufficiently small, $z_{j} \in C^{\infty}\left(\mathcal{U}^{1,0}, \mathbb{C}\right)$. The following formulas hold, summing over repeated index a:

$$
\begin{aligned}
\nabla z_{j}= & -\left\langle\sigma_{3} \xi_{j} \mid \partial_{\omega} R\right\rangle \nabla \omega-\mathrm{i}\left\langle\sigma_{3} \xi_{j} \mid \sigma_{3} R\right\rangle \nabla \vartheta-\frac{\mathrm{i}}{2}\left\langle\sigma_{3} \xi_{j} \mid \sigma_{3} x_{a} R\right\rangle \nabla v_{a} \\
& +\left\langle\sigma_{3} \xi_{j} \mid\left(\partial_{x_{a}}+\mathrm{i} \sigma_{3} \frac{v_{a}}{2}\right) R\right\rangle \nabla D_{a}+\tau_{D}\left(e^{-\mathrm{i} \sigma_{3} \Theta} \sigma_{3} \xi_{j}\right)(x), \\
\nabla \bar{z}_{j}= & -\left\langle\sigma_{1} \sigma_{3} \xi_{j} \mid \partial_{\omega} R\right\rangle \nabla \omega-\mathrm{i}\left\langle\sigma_{1} \sigma_{3} \xi_{j} \mid \sigma_{3} R\right\rangle \nabla \vartheta-\frac{\mathrm{i}}{2}\left\langle\sigma_{1} \sigma_{3} \xi_{j} \mid \sigma_{3} x_{a} R\right\rangle \nabla v_{a} \\
& +\left\langle\sigma_{1} \sigma_{3} \xi_{j} \mid\left(\partial_{x_{a}}+\mathrm{i} \sigma_{3} \frac{v_{a}}{2}\right) R\right\rangle \nabla D_{a}+\tau_{D}\left(e^{-\mathrm{i} \sigma_{3} \Theta} \sigma_{1} \sigma_{3} \xi_{j}\right)(x) .
\end{aligned}
$$

Proof. The fact that $z_{j} \in C^{\infty}\left(\mathcal{U}^{1,0}, \mathbb{C}\right)$ follows from the formula

$$
z_{j}(U)=\left\langle U(x), e^{-\mathrm{i} \sigma_{3}\left(\frac{v \cdot(x-D)}{2}+\vartheta\right)} \sigma_{3} \xi_{j}(\omega, x-D)\right\rangle,
$$

the fact that $\omega, \vartheta, v, D \in C^{\infty}\left(\mathcal{U}^{1,0}\right)$ and the properties of $\xi_{j}(\omega, x)$ we have

$$
\begin{aligned}
& \left\langle\nabla z_{j} \mid \tau_{D} e^{\mathrm{i} \sigma_{3} \Theta} \xi_{\ell}\right\rangle=\delta_{j \ell}, \quad\left\langle\nabla z_{j} \mid \tau_{D} e^{\mathrm{i} \sigma_{3} \Theta} \sigma_{1} \xi_{\ell}\right\rangle=0=\left\langle\nabla z_{j} \mid \tau_{D} e^{\mathrm{i} \sigma_{3} \Theta} \sigma_{3}(\Phi+R)\right\rangle, \\
& \left\langle\nabla z_{j} \mid \tau_{D} e^{\mathrm{i} \sigma_{3} \Theta} \partial_{\omega}(\Phi+R)\right\rangle=0=\left\langle\nabla z_{j}, \tau_{D} e^{\sigma_{3} \Theta} x_{a}(\Phi+R)\right\rangle, \\
& \left\langle\nabla z_{j} \mid \tau_{D} e^{\sigma_{3} \Theta}\left(\partial_{x_{a}}+\mathrm{i} \sigma_{3} \frac{v_{a}}{2}\right)(\Phi+R)\right\rangle=0 \\
& \left\langle\nabla z_{j} \mid \tau_{D} e^{\mathrm{i} \sigma_{3} \Theta} P_{c}(\omega) P_{c}\left(\omega_{0}\right) g\right\rangle=0 \quad \forall g \in L_{c}^{2}\left(\mathcal{H}_{\omega_{0}}\right) .
\end{aligned}
$$

The latter implies $P_{c}\left(\mathcal{H}_{\omega_{0}}^{*}\right) P_{c}\left(\mathcal{H}_{\omega}^{*}\right) e^{\mathrm{i} \sigma_{3} \Theta} \tau_{-D} \nabla z_{j}=0$ and $P_{c}\left(\mathcal{H}_{\omega}^{*}\right) e^{\mathrm{i} \sigma_{3} \Theta} \tau_{-D} \nabla z_{j}=0$. By (2.10) and for $\mathbf{A}$ and an unknown vector,

$$
\nabla z_{j}=\mathbf{A}^{T}\left(\begin{array}{c}
\tau_{D}\left(e^{-\mathrm{i} \sigma_{3} \Theta} \Phi\right)(x) \\
\tau_{D}\left(e^{-\mathrm{i} \sigma_{3} \Theta} \sigma_{3} \partial_{\omega} \Phi\right)(x) \\
\tau_{D}\left(e^{-\mathrm{i} \sigma_{3} \Theta} x \Phi\right)(x) \\
\tau_{D}\left(e^{-\mathrm{i} \sigma_{3} \Theta} \sigma_{3} \nabla_{x} \Phi\right)(x)
\end{array}\right)+\tau_{D}\left(e^{-\mathrm{i} \sigma_{3} \Theta} \sigma_{3} \xi_{j}\right)(x)
$$

Using Lemma 2.2 there is a vector $\mathbf{A}_{1}$ s.t.

$$
\nabla z_{j}=\mathbf{A}_{1}^{T}\left(\begin{array}{c}
\nabla \omega \\
\nabla \vartheta \\
\nabla v \\
\nabla D
\end{array}\right)+\tau_{D}\left(e^{-\mathrm{i} \sigma_{3} \Theta} \sigma_{3} \xi_{j}\right)(x) .
$$

By (2.16) and (2.20) we obtain

$$
\mathbf{A}_{1}=-\left(\begin{array}{c}
\left\langle\sigma_{3} \xi_{j} \mid \partial_{\omega} R\right\rangle \\
\mathrm{i}\left\langle\sigma_{3} \xi_{j} \mid \sigma_{3} R\right\rangle \\
\frac{\mathrm{i}}{2}\left\langle\sigma_{3} \xi_{j} \mid \sigma_{3} x R\right\rangle \\
-\left\langle\sigma_{3} \xi_{j} \mid\left(\nabla_{x}+\mathrm{i} \sigma_{3} \frac{v}{2}\right) R\right\rangle
\end{array}\right)
$$

Similar formulas hold for $\nabla \bar{z}_{j}$ yielding Lemma 2.3

Lemma 2.4. The map $U \rightarrow f(U)=f$, for $U$ and $f$ as in (2.14), is continuous from $X$ into itself, for $X=L^{2}, H^{1}$ and $\Sigma_{n}$ for any $n$. Furthermore we have $f \in C^{1}\left(\mathcal{U}^{1,0}, L^{2,-1}\right), f \in C^{1}\left(\mathcal{U}^{1,0} \cap \Sigma, L^{2}\right)$ and $f \in C^{1}\left(\mathcal{U}^{1,0} \cap \Sigma_{n}, \Sigma_{n-1}\right)$ with Fréchet derivative $f^{\prime}(U)$ defined by the following formula, summing on the repeated 
index a:

$$
\begin{aligned}
& f^{\prime}(U)=\left(P_{c}(\omega) P_{c}\left(\omega_{0}\right)\right)^{-1} P_{c}(\omega)\left[-\partial_{\omega} R d \omega\right. \\
& \left.\quad-\mathrm{i} \sigma_{3} R d \vartheta-\frac{\mathrm{i}}{2} \sigma_{3} x_{a} R d v_{a}+\left(\partial_{x_{a}}+\mathrm{i} \sigma_{3} \frac{v_{a}}{2}\right) R d D_{a}+e^{-\mathrm{i} \sigma_{3} \Theta} \tau_{-D} \mathbb{1}\right] .
\end{aligned}
$$

We further have $f \in C^{n}\left(\mathcal{U}^{1,0} \cap \Sigma, H^{-n+1,-n}\right)$.

Proof. Continuity follows from Lemmas 2.1]2.3 and formula (2.14) solved w.r.t. $f$. The latter also proves the $C^{1}$ as well as the $C^{n}$ properties. The proof of formula (2.21) is similar to that of Lemma 2.3. see also Lemma 4.2 [Cu1].

In the sequel, given a scalar function $\psi(U)$ which is differentiable, we will denote by $\nabla_{f} \psi(U)$ the only element in $L_{c}^{2}\left(\mathcal{H}_{\omega_{0}}^{*}\right)$ s.t. for any $g \in L_{c}^{2}\left(\mathcal{H}_{\omega_{0}}\right)$ we have $\left\langle\nabla_{f} \psi(U) \mid g\right\rangle=\left\langle\nabla \psi(U) \mid P_{c}^{*}(\omega) g\right\rangle$.

\section{Symplectic structure}

Our ambient space is $\mathbf{X} \times \mathbf{X}$, where we can have $\mathbf{X}=L^{2}, H^{1}, \Sigma_{n}$. We focus only on points with $\sigma_{1} U=\bar{U}$, so that the space is identified with $\mathbf{X}$. The natural symplectic structure for our problem is

$$
\Omega(X, Y)=\left\langle X \mid \sigma_{3} \sigma_{1} Y\right\rangle .
$$

Definition 3.1. Let $F \in C^{1}(\mathcal{U}, \mathbb{C})$ for $\mathcal{U}$ and an open subset of $\mathbf{X}$. Then the Hamiltonian vectorfield of $F$ with respect to a symplectic form $\Omega$ is the field $X_{F}$ such that $\Omega\left(X_{F}, Y\right)=-\mathrm{i} d F(Y)$ for any given tangent vector $Y \in T \mathcal{U}$. More explicitly, $X_{F}=-\mathrm{i} \sigma_{3} \sigma_{1} \nabla F$ for the form in (3.1).

Definition 3.2. For $F, G \in C^{1}(\mathcal{U}, \mathbb{C})$ as above, we call a Poisson bracket of a pair of $F$ and $G$ the function

$$
\{F, G\}=d F\left(X_{G}\right)=\mathrm{i} \Omega\left(X_{F}, X_{G}\right)=-\mathrm{i}\left\langle\nabla F \mid \sigma_{3} \sigma_{1} \nabla G\right\rangle .
$$

Let $\mathcal{G} \in C^{1}(\mathcal{U}, \mathbb{E})$ with $\mathbb{E}$ a given Banach space on $\mathbb{C}$. Then, for $F \in C^{1}(\mathcal{U}, \mathbb{C})$ we set, for $\mathcal{G}^{\prime}$ the Fréchet derivative of $\mathcal{G}$,

$$
\{\mathcal{G}, F\}:=\mathcal{G}^{\prime}(U) X_{F}(U)=-\mathrm{i} \mathcal{G}^{\prime}(U) \sigma_{3} \sigma_{1} \nabla F(U) .
$$

We set $\{F, \mathcal{G}\}:=-\{\mathcal{G}, F\}$.

Obviously our system is Hamiltonian. It is important to cast it in terms of the Poisson brackets.

Lemma 3.3. In the coordinate system (2.14), system (2.6) can be written, for $F=E$, as $\dot{\vartheta}=\{\vartheta, F\}$ and

$$
\begin{aligned}
& \dot{\omega}=\{\omega, F\}, \quad \dot{f}=\{f, F\}, \\
& \dot{D}_{a}=\left\{D_{a}, F\right\}, \quad \dot{v}_{a}=\left\{v_{a}, F\right\} \text { for } a=1,2,3, \\
& \dot{z}_{j}=\left\{z_{j}, F\right\}, \quad \dot{\bar{z}}_{j}=\left\{\bar{z}_{j}, F\right\} \text { for } j=1, \ldots, m .
\end{aligned}
$$

Proof. The statement is not standard, only for $\dot{f}=\{f, E\}$. Notice that (2.6) can be written as

$$
\begin{aligned}
& \mathrm{i} \dot{U}=-\sigma_{3}\left(\dot{\vartheta}+\frac{\dot{v} \cdot(x-D)}{2}\right) U+\mathrm{i} \dot{\omega} e^{\mathrm{i} \sigma_{3} \Theta} \partial_{\omega}(\Phi+R) \\
& -\mathrm{i} \dot{D} \cdot \nabla\left({ }^{\mathrm{i} \sigma_{3} \Theta}(\Phi+R)\right)+\mathrm{i} e^{\mathrm{i} \sigma_{3} \Theta}\left(\dot{z} \cdot \xi+\dot{\bar{z}} \cdot \sigma_{1} \xi+P_{c}\left(\mathcal{H}_{\omega}\right) \dot{f}\right) .
\end{aligned}
$$


So, by (2.16), system i $\dot{U}=\sigma_{3} \sigma_{1} \nabla E(U)$ is the same as

$$
\begin{aligned}
& \mathrm{i} \dot{\vartheta} \frac{\partial}{\partial \vartheta}+\sum_{a=1}^{3} \dot{v}_{a} \frac{\partial}{\partial v_{a}}+\mathrm{i} \dot{\omega} \frac{\partial}{\partial \omega}+\sum_{a=1}^{3} \dot{D}_{a} \frac{\partial}{\partial D_{a}}+\mathrm{i} \sum_{j=1}^{m} \dot{z}_{j} \frac{\partial}{\partial z_{j}}+\mathrm{i} \sum_{j=1}^{m} \dot{\bar{z}}_{j} \frac{\partial}{\partial \bar{z}_{j}} \\
& +\mathrm{i} e^{\mathrm{i} \Theta \sigma_{3}} P_{c}\left(\mathcal{H}_{\omega}\right) \dot{f}=\sigma_{3} \sigma_{1} \nabla E(U) .
\end{aligned}
$$

When we apply the derivative $f^{\prime}(U)$ to (3.6) the first line cancels, so we get

$$
f^{\prime}(U) e^{\mathrm{i} \Theta \sigma_{3}} P_{c}\left(\mathcal{H}_{\omega}\right) \dot{f}=-f^{\prime}(U) \mathrm{i} \sigma_{3} \sigma_{1} \nabla E(U)=f^{\prime}(U) X_{E}(U)=\{f, E\}
$$

from the definition of a Hamiltonian field and of a Poisson bracket. Notice now that $\dot{f}=f^{\prime}(U) e^{\mathrm{i} \Theta \sigma_{3}} P_{c}\left(\mathcal{H}_{\omega}\right) \dot{f}$. This follows from the fact that

$$
\begin{aligned}
& f^{\prime}(U) e^{\mathrm{i} \Theta \sigma_{3}} P_{c}\left(\mathcal{H}_{\omega}\right) \dot{f}=\left.\frac{d}{d s}\right|_{s=0} f(U(\omega, \vartheta, D, v, z, \bar{z}, f+s \dot{f})) \\
& =\left.\frac{d}{d s}\right|_{s=0}(f+s \dot{f})=\dot{f} .
\end{aligned}
$$

Hence $\dot{f}=\{f, E\}$.

\section{Reduction of VARIABLES}

The following formulas are important.

Lemma 4.1. Consider charge $Q$ and momenta $\Pi_{a}$, as defined in (2.3). Then

$$
X_{Q}=-\frac{\partial}{\partial \vartheta}, \quad X_{\Pi_{a}}=-\frac{\partial}{\partial D_{a}}
$$

In particular

$$
\{Q, \vartheta\}=\left\{\Pi_{a}, D_{a}\right\}=1
$$

and any Poisson bracket not in (4.2) of any of the invariants $Q, \Pi_{a}$ with any of the coordinates from (2.14) is equal to 0 . The functions $Q, \Pi_{a}$ and $E$ Poisson commute.

Proof. (4.1) follows from (2.16):

$$
\begin{aligned}
& X_{Q}(U)=-\mathrm{i} \sigma_{3} \sigma_{1} \nabla Q(U)=-\mathrm{i} \sigma_{3} U=-\frac{\partial}{\partial \vartheta}, \\
& X_{\Pi_{a}}(U)=-\mathrm{i} \sigma_{3} \sigma_{1} \nabla \Pi_{a}(U)=\mathrm{i} \sigma_{3} \sigma_{1} \sigma_{2} \partial_{a} U=\partial_{a} U=-\frac{\partial}{\partial D_{a}} .
\end{aligned}
$$

The second part of the statement follows immediately from (4.1).

The following lemma is elementary.

Lemma 4.2. For $U=e^{\mathrm{i} \sigma_{3}\left(\frac{v \cdot(x-D)}{2}+\vartheta\right)}\left(\Phi_{\omega}(x-D)+R(x-D)\right)$ and summing on repeated indexes, we have the following formulas:

$$
\begin{aligned}
& Q(U)=Q\left(\Phi_{\omega}+R\right)=q(\omega)+Q(R), \\
& \Pi_{a}(U)=\Pi_{a}\left(\Phi_{\omega}+R\right)-2^{-1} v_{a} Q\left(\Phi_{\omega}+R\right), \\
& E(U)=E\left(\Phi_{\omega}+R\right)-v_{a} \Pi_{a}\left(\Phi_{\omega}+R\right)+\frac{v^{2}}{4} Q\left(\Phi_{\omega}+R\right) .
\end{aligned}
$$

We have $\Pi_{a}\left(\Phi_{\omega}+R\right)=\Pi_{a}(R) . Q(U)$ and $\Pi_{a}(U)$ are $C^{\infty}$ and $E(U)$ is $C^{1}$ in $\omega$, $v, z$ and $f \in H^{1}$. Furthermore both $E$ and $\nabla E$ depend smoothly on $(\omega, z)$. 
Now we introduce a new Hamiltonian for $U_{0}^{T}=\left(u_{0}, \bar{u}_{0}\right)$,

$$
K(U):=E(U)-E\left(\Phi_{\omega_{0}}\right)+\omega(U)\left(Q(U)-Q\left(U_{0}\right)\right) .
$$

Lemma 4.1 implies that the solutions of (2.6) with charge $q\left(\omega_{0}\right)$ satisfy (3.4) with $F=K$ and $\dot{\vartheta}-\omega=\{\vartheta, K\}$. We would like $K$ to be our Hamiltonian, but obviously $K$ is not a Hamiltonian for (2.6). To obviate this we notice that $\partial_{D_{a}} K=\partial_{\vartheta} K \equiv 0$ implies that the evolution of the variables $\omega, v_{a}, z_{j}, \bar{z}_{j}, f$ is unchanged if we consider the following new Hamiltonian system:

$$
\begin{aligned}
& \dot{\omega}=\{\omega, K\}, \quad \dot{\vartheta}=\{\vartheta, K\}, \quad \dot{f}=\{f, K\}, \\
& \dot{D}_{a}=\left\{D_{a}, K\right\}, \quad \dot{v}_{a}=\left\{v_{a}, K\right\} \text { for } a=1,2,3, \\
& \dot{z}_{j}=\left\{z_{j}, K\right\}, \quad \dot{\bar{z}}_{j}=\left\{\bar{z}_{j}, K\right\} \text { for } j=1, \ldots, m .
\end{aligned}
$$

It is elementary that for (4.4) the charge $Q(U)$ and the momenta $\Pi_{a}(U)$ are invariants of motion.

We proceed to a reduction of order in (4.4) such as described for instance in Theorem 6.35 , p. 402 of $[\mathrm{O}$. Here the discussion is elementary because we have no need to prove the existence of particular variables; see p. 395 of [O]. We set

$$
\begin{aligned}
& Q:=Q(U)=q(\omega)+Q(R), \\
& \Pi_{a}:=\Pi_{a}(U)=\Pi_{a}(R)-\frac{v_{a}}{2}(q(\omega)+Q(R)) .
\end{aligned}
$$

Lemma 4.3. After we express $R$ in (4.5) in terms of $\omega, z$ and $f$ (see (2.13) ), there is an implicit function $\omega=\omega(Q, z, f)$ defined by the first of (4.5), with $\omega(Q, z, f)$ smooth in $Q, z$ and in $f \in L_{c}^{2}\left(\omega_{0}\right)$. Similarly, $v_{a}=v_{a}\left(\Pi_{a}, Q, z, f\right)$, with the latter smooth in $\Pi_{a}, Q, z$ and $f \in H_{c}^{\frac{1}{2}}\left(\omega_{0}\right)$.

Proof. For $\omega=\omega(Q, z, f)$ the statement follows from the implicit function theorem. Write $v_{a}=2 Q^{-1}\left(\Pi_{a}(R)-\Pi_{a}\right)$ and substitute $\omega=\omega(Q, z, f)$ in the definition of $R$.

Lemma 4.3 allows us to move from the variables on the rhs of (2.14) to a new system of variables obtained by replacing the functions $(\omega, v)$ with the $(Q, \Pi)$.

Lemma 4.4. The vectorfields $\frac{\partial}{\partial \vartheta}$ and $\frac{\partial}{\partial D_{a}}$ are the same for the two systems of coordinates. In particular, in the new system of coordinates we continue to have $\frac{\partial}{\partial \vartheta} K=\frac{\partial}{\partial D_{a}} K=0$

Proof. It is an immediate consequence of $\frac{\partial}{\partial \vartheta} Q(U)=\frac{\partial}{\partial D_{a}} Q(U)=0$ and of $\frac{\partial}{\partial \vartheta} \Pi_{b}(U)$ $=\frac{\partial}{\partial D_{a}} \Pi_{b}(U)=0$ in the old coordinate system, and of the chain rule.

In the new variables, system (4.4) reduces to the pair of systems

$$
\dot{Q}=0, \quad \dot{\vartheta}=\{\vartheta, K\}, \quad \dot{D}_{a}=\left\{D_{a}, K\right\}, \quad \dot{\Pi}_{a}=0 \quad \text { for } a=1,2,3,
$$

and

$$
\dot{f}=\{f, K\}, \quad \dot{z}_{j}=\left\{z_{j}, K\right\}, \quad \dot{\bar{z}}_{j}=\left\{\bar{z}_{j}, K\right\} \quad \text { for } j=1, \ldots, m .
$$

Now we restrict our attention to the set with $Q=Q\left(U_{0}\right)$ and $\Pi_{a}=0$. Thanks to Lemma 4.4 system (4.7) is closed. 
Lemma 4.5. Consider the restriction of the variables $(Q, \Pi)$ at the fixed values $Q=Q\left(U_{0}\right)$ and $\Pi_{a}=0$ and set $\varrho(f):=(Q(f), \Pi(f))$. Then we have the expansion

$$
K=\underline{\psi}(\varrho(f))+K_{2}+\underline{\mathcal{R}}^{(1)},
$$

where $\underline{\psi}(\varrho)$ is smooth in $\varrho$ and where the following holds:

(1) We have

$$
K_{2}=\sum_{\substack{|\mu+\nu|=2 \\ \lambda^{0} \cdot(\mu-\nu)=0}} \underline{a}_{\mu \nu}(\varrho(f)) z^{\mu} \bar{z}^{\nu}+\frac{1}{2}\left\langle\sigma_{3} \mathcal{H}_{\omega_{0}} f \mid \sigma_{1} f\right\rangle .
$$

(2) We have $\underline{\mathcal{R}}^{(1)}=\widetilde{\mathcal{\mathcal { R }}}^{(1)}+\widetilde{\mathcal{\mathcal { R }}^{(2)}}$, with

$$
\begin{gathered}
\widetilde{\mathcal{R}^{(1)}}=\sum_{\substack{|\mu+\nu|=2 \\
\lambda^{0} \cdot(\mu-\nu) \neq 0}} \underline{a}_{\mu \nu}(\varrho(f)) z^{\mu} \bar{z}^{\nu}+\sum_{|\mu+\nu|=1} z^{\mu} \bar{z}^{\nu}\left\langle\sigma_{1} \sigma_{3} \underline{G}_{\mu \nu}(\varrho(f)) \mid f\right\rangle, \\
\widetilde{\mathcal{\mathcal { R }}^{(2)}}=\sum_{|\mu+\nu|=3} z^{\mu} \bar{z}^{\nu} \underline{a}_{\mu \nu}(z, \varrho(f))+\sum_{|\mu+\nu|=2} z^{\mu} \bar{z}^{\nu}\left\langle\underline{G}_{\mu \nu}(z, \varrho(f)) \mid \sigma_{3} \sigma_{1} f\right\rangle \\
+\sum_{d=2}^{4}\left\langle\underline{B}_{d}(z, \varrho(f)) \mid f^{d}\right\rangle+\int_{\mathbb{R}^{3}} \underline{B}_{5}(x, z, f(x), \varrho(f)) f^{5}(x) d x+E_{P}(f),
\end{gathered}
$$

with $\underline{B}_{2}(0,0)=0$ and where, both here and in Theorem 12.1 later, by $f^{d}(x)$ we schematically represent $d$-products of components of $f$.

(3) At $\varrho(f)=0$,

$$
\begin{aligned}
& \underline{a}_{\mu \nu}(0)=0 \text { for }|\mu+\nu|=2 \text { with }(\mu, \nu) \neq\left(\delta_{j}, \delta_{j}\right) \text { for all } j, \\
& \underline{a}_{\delta_{j} \delta_{j}}^{(r)}(0)=\lambda_{j}\left(\omega_{0}\right) \text {, where } \delta_{j}=\left(\delta_{1 j}, \ldots, \delta_{m j}\right), \\
& \underline{G}_{\mu \nu}(0)=0 \text { for }|\mu+\nu|=1 .
\end{aligned}
$$

These $\underline{a}_{\mu \nu}(\varrho)$ and $\underline{G}_{\mu \nu}(x, \varrho)$ are smooth in all variables with $G_{\mu \nu}(\cdot, \varrho) \in$ $C^{\infty}\left(\mathbb{R}^{4}, H_{x}^{K, S}\left(\mathbb{R}^{3}, \mathbb{C}^{2}\right)\right)$ for all $(K, S)$.

(4) $\underline{a}_{\mu \nu}(z, \varrho) \in C^{\infty}(\mathrm{U}, \mathbb{C})$ for a small neighborhood $\mathrm{U}$ of $(0,0)$ in $\mathbb{C}^{m} \times \mathbb{R}^{4}$.

(5) $\underline{G}_{\mu \nu}(\cdot, z, \varrho) \in C^{\infty}\left(\mathrm{U}, H_{x}^{K, S}\left(\mathbb{R}^{3}, \mathbb{C}^{2}\right)\right)$, for $\mathrm{U}$ as in (4) and for all $(K, S)$.

(6) $\underline{B}_{d}(\cdot, z, \varrho) \in C^{\infty}\left(\mathrm{U}, H_{x}^{K, S}\left(\mathbb{R}^{3}, B\left(\left(\mathbb{C}^{2}\right)^{\otimes d}, \mathbb{C}\right)\right)\right)$, for $2 \leq d \leq 4$ for $\mathrm{U}$ and $(K, S)$ as above.

(7) Let ${ }^{t} \eta=(\zeta, \bar{\zeta})$ for $\zeta \in \mathbb{C}$. Then for $\underline{B}_{5}(\cdot, \omega, z, \eta, \varrho)$ we have for all $(K, S)$

for any $l,\left\|\nabla_{z, \bar{z}, \zeta, \bar{\zeta}, \varrho}^{l} \underline{B}_{5}(z, \eta, \varrho)\right\|_{H_{x}^{K, S}\left(\mathbb{R}^{3}, B\left(\left(\mathbb{C}^{2}\right) \otimes 5, \mathbb{C}\right)\right)} \leq C_{l}(K, S)$.

(8) For all indexes and for $b_{\mu \nu}=\underline{a}_{\mu \nu}$ and $B_{\mu \nu}=\underline{G}_{\mu \nu}$, we have

$$
b_{\mu \nu}=\overline{b_{\nu \mu}}, \quad B_{\mu \nu}=-\sigma_{1} \overline{B_{\nu \mu}} .
$$

(9) $\underline{B}_{5}$ depends on $f(x)$ with terms $|f(x)|^{2}$ and $\varphi(x) f(x)$, with $\varphi(x)$ Schwartz functions.

Proof. By Lemma 4.2 we get from (4.3), for $d(\omega)$ and $q(\omega)$ (see (2.5)),

$$
\begin{aligned}
K(U) & =E\left(\Phi_{\omega}+R\right)+\omega Q\left(\Phi_{\omega}+R\right)-d\left(\omega_{0}\right)-\left(\omega-\omega_{0}\right) q\left(\omega_{0}\right) \\
& +\frac{v^{2}}{4} Q\left(\Phi_{\omega}+R\right)-v_{a} \Pi_{a}(R) .
\end{aligned}
$$


We have

$$
E\left(\Phi_{\omega}+R\right)+\omega Q\left(\Phi_{\omega}+R\right)=d(\omega)+\frac{1}{2}\left\langle\sigma_{3} \mathcal{H}_{\omega} R \mid \sigma_{1} R\right\rangle+\widehat{\mathcal{R}}^{(1)}
$$

with an expansion of the following form:

$$
\begin{aligned}
& \widehat{\mathcal{R}}^{(1)}=\sum_{|\mu+\nu|=3} z^{\mu} \bar{z}^{\nu} \underline{a}_{\mu \nu}(\omega, z)+\sum_{|\mu+\nu|=2} z^{\mu} \bar{z}^{\nu}\left\langle\underline{G}_{\mu \nu}(\omega, z) \mid \sigma_{3} \sigma_{1} P_{c}(\omega) f\right\rangle \\
& +\sum_{d=2}^{4}\left\langle\underline{B}_{d}(\omega, z) \mid f^{d}\right\rangle+\int_{\mathbb{R}^{3}} \underline{B}_{5}(x, \omega, z, f(x)) f^{5}(x) d x+E_{P}\left(P_{c}(\omega) f\right) .
\end{aligned}
$$

The coefficients in the expansion of $\widehat{\mathcal{R}}^{(1)}$ satisfy appropriate smoothness and symmetry properties. When we restrict ourselves to $Q=Q\left(U_{0}\right)$, by $q(\omega)+Q(R)=q\left(\omega_{0}\right)$ we get that $\omega-\omega_{0}=O\left(|z|^{2}+\|f\|_{2}^{2}+\|f\|_{H^{-K,-S}}^{2}\right)$. Notice that we can express the function $\omega(Q, z, f)$ as a function $\omega\left(Q, z, f, \varrho_{0}(f)\right)$, with $\varrho_{0}(f)=Q(f)$, such that $\omega\left(Q, z, f, \varrho_{0}\right)$ is smooth in the variables $z \in \mathbb{C}^{m}, f \in H^{-K,-S}$ and $Q, \varrho_{0} \in \mathbb{R}$. Hence $\widehat{\mathcal{R}}^{(1)}$ is a sum of terms which can be absorbed in $\widetilde{\mathcal{R}^{(2)}}$, with dependence on $\varrho(f)=(Q(f), \Pi(f))$ reduced to that of $Q(f)$. We have by $d^{\prime}=q$

$$
d(\omega)-d\left(\omega_{0}\right)-\left(\omega-\omega_{0}\right) q\left(\omega_{0}\right)=\frac{1}{2} d^{\prime \prime}\left(\omega_{0}\right)\left(\omega-\omega_{0}\right)^{2}+o\left(\omega-\omega_{0}\right)^{2} .
$$

So (4.15) can be absorbed partly in $\underline{\psi}(\varrho(f))$ and partly in the rest of expansion (4.8). We have

$$
\begin{aligned}
\frac{1}{2}\left\langle\sigma_{3} \mathcal{H}_{\omega} R \mid \sigma_{1} R\right\rangle & =\frac{1}{2}\left\langle\sigma_{3} \mathcal{H}_{\omega_{0}} R \mid \sigma_{1} R\right\rangle \\
& +\left(\omega-\omega_{0}\right) Q(R)+\frac{1}{2}\left\langle\sigma_{3}\left(V_{\omega}-V_{\omega_{0}}\right) R \mid \sigma_{1} R\right\rangle,
\end{aligned}
$$

where $\frac{1}{2}\left\langle\sigma_{3} \mathcal{H}_{\omega_{0}} R \mid \sigma_{1} R\right\rangle=\sum_{j} \lambda_{j}\left(\omega_{0}\right)\left|z_{j}\right|^{2}+\frac{1}{2}\left\langle\sigma_{3} \mathcal{H}_{\omega_{0}} f \mid \sigma_{1} f\right\rangle$. In the last line of (4.16), the first term is similar to (4.15) and the second can be absorbed in $H_{2}^{(1)}+\mathcal{R}^{(1)}$. Since we have $v_{a} q\left(\omega_{0}\right)=2 \Pi_{a}(R)$, we also get that the second line of (4.13) can be decomposed into terms with the properties of the various terms on the rhs of (4.8).

\section{A vectorfield needed for the Darboux Theorem}

We introduce the 2-form, summing on repeated indexes:

$$
\Omega_{0}=\mathrm{i} d \vartheta \wedge d Q+\mathrm{i} d D_{a} \wedge d \Pi_{a}+d z_{j} \wedge d \bar{z}_{j}+\left\langle f^{\prime} \mid \sigma_{3} \sigma_{1} f^{\prime}\right\rangle .
$$

Through a change of variable we will transform the $\Omega$ defined in (3.1) into the $\Omega_{0}$. A brief reminder of J. Moser's scheme of proof is in Sect. 7 of [Cu1 and will not be repeated here. The first step in the implementation of this scheme is the search of an appropriate vectorfield $\mathcal{X}^{t}$, which satisfies an equation stated in Lemma 5.2 . Then we will have $\phi^{*} \Omega=\Omega_{0}$, with $\phi=\phi^{1}$ the Lie transform associated to $\phi^{t}$, the flow of $\mathcal{X}^{t}$. Existence and differentiability of $\phi^{t}$ are not obvious. Lemma 5.8 will tell us that to get $\phi^{t}$ we need information on a quasilinear hyperbolic symmetric system mixed to ODE's for the discrete modes. In Lemma 5.8 we will establish some properties of the coefficients of the system. Sections 6 , 8 contain material needed to establish existence and differentiability of $\phi^{t}$. Lemma 5.1 is similar to Lemma 7.2 of $\mathrm{Cu} 1$. 
Lemma 5.1. At the points $\tau_{D} e^{\mathrm{i} \sigma_{3}\left(\frac{v \cdot x}{2}+\vartheta\right)} \Phi_{\omega_{0}}$ for all $(\vartheta, D, v) \in \mathbb{R}^{7}$ we have $\Omega_{0}=\Omega$. Consider the following forms:

$$
\mathrm{B}_{0}:=\mathrm{i} \vartheta d Q+\mathrm{i} D_{a} d \Pi_{a}-\frac{\bar{z}_{j} d z_{j}-z_{j} d \bar{z}_{j}}{2}+\frac{1}{2}\left\langle f \mid \sigma_{3} \sigma_{1} f^{\prime}\right\rangle
$$

and $\mathrm{B}:=\mathrm{B}_{0}+\Gamma$ for $\Gamma=\varphi d Q+(\Gamma)_{j} d z_{j}+(\Gamma)_{\bar{j}} d \bar{z}_{j}+\left\langle(\Gamma)_{f} \mid f^{\prime}\right\rangle$, where

$$
\begin{aligned}
& \varphi:=\frac{\left\langle\sigma_{1} \sigma_{3} R \mid \partial_{\omega} R\right\rangle+\mathrm{i} v_{a}\left\langle x_{a} \sigma_{1} R \mid \partial_{\omega} R\right\rangle}{2\left(q^{\prime}+\partial_{\omega} Q(R)\right)}, \\
& (\Gamma)_{j}:=\frac{\mathrm{i}}{2} v_{a}\left\langle x_{a} \sigma_{1} R \mid \xi_{j}\right\rangle-\varphi \partial_{j} Q(R),(\Gamma)_{\bar{j}}:=\frac{\mathrm{i}}{2} v_{a}\left\langle x_{a} R \mid \xi_{j}\right\rangle-\varphi \partial_{j} Q(R), \\
& (\Gamma)_{f}:=P_{c}^{*}\left(\omega_{0}\right)\left(\frac{\mathrm{i}}{2} v_{a} P_{c}^{*}(\omega) \sigma_{1} x_{a} R-\frac{1}{2} \sigma_{1} \sigma_{3} P_{d}(\omega) f-\varphi P_{c}^{*}(\omega) \sigma_{1} R\right) .
\end{aligned}
$$

Then $d \mathrm{~B}_{0}=\Omega_{0}$ and $d \mathrm{~B}=\Omega$.

Proof. $d \mathrm{~B}_{0}=\Omega_{0}$ follows from the definition of an exterior differential. Since $|\varphi| \leq$ $C\left(|z|+\|f\|_{H^{-K,-S}}\right)^{2}$ and since $P_{d}(\omega) f=\left(P_{d}(\omega)-P_{d}\left(\omega_{0}\right)\right) f$ is a 0 of order 2 at $\omega=\omega_{0}$ and $f=0$, to get $d \Gamma=0$ at $R=0$ and $\omega=\omega_{0}$ it is enough to show that

$$
d\left(\left\langle x_{a} \sigma_{1} R \mid \xi_{j}\right\rangle d z_{j}+\left\langle x_{a} R \mid \xi_{j}\right\rangle d \bar{z}_{j}+\left\langle P_{c}^{*}(\omega) \sigma_{1} x_{a} R \mid f^{\prime}\right\rangle\right)=0 \text { at } R=0 .
$$

But this is true. Formally $d\left(d\left\langle x_{a} \sigma_{1} R \mid R\right\rangle-\partial_{\omega}\left\langle x_{a} \sigma_{1} R \mid R\right\rangle d \omega\right)=0$ at $R=0$.

We prove the formula for B. Set $\widetilde{B}:=\frac{1}{2}\left\langle\sigma_{1} \sigma_{3} U\right|$. Notice that $d \widetilde{B}=\Omega$. We get

$$
\begin{aligned}
& \widetilde{B}=\frac{1}{2}\left\langle\tau_{D} e^{-\mathrm{i} \sigma_{3} \Theta} \sigma_{1} \sigma_{3} \Phi\right|+\frac{1}{2}\left\langle\tau_{D} e^{-\mathrm{i} \sigma_{3} \Theta} \sigma_{1} \sigma_{3} P_{c}(\omega) f\right| \\
& +\frac{1}{2} z_{j}\left\langle\tau_{D} e^{-\mathrm{i} \sigma_{3} \Theta} \sigma_{1} \sigma_{3} \xi_{j}\right|-\frac{1}{2} \bar{z}_{j}\left\langle\tau_{D} e^{-\mathrm{i} \sigma_{3} \Theta} \sigma_{3} \xi_{j}\right|,
\end{aligned}
$$

using (2.14). By Lemmas 2.3 and 2.4, the sum of the last three terms equals

$$
\begin{aligned}
& \frac{1}{2} z_{j} d \bar{z}_{j}-\frac{1}{2} \bar{z}_{j} d z_{j}+\frac{1}{2}\left\langle\sigma_{1} \sigma_{3} f \mid P_{c}(\omega) f^{\prime}\right\rangle+\frac{1}{2}\left\langle\sigma_{1} \sigma_{3} R \mid \partial_{\omega} R\right\rangle d \omega \\
& -\mathrm{i} Q(R) d \vartheta-\frac{\mathrm{i}}{4}\left\langle\sigma_{1} R \mid x_{a} R\right\rangle d v_{a}-\mathrm{i}\left(\Pi_{a}(R)-\frac{v_{a}}{2} Q(R)\right) d D_{a} .
\end{aligned}
$$

Applying $\frac{1}{2} \tau_{D} e^{-\mathrm{i} \sigma_{3} \Theta} \sigma_{1} \sigma_{3}$ to decomposition (2.10) for $X=\Phi$, we get

$$
\begin{aligned}
& \frac{1}{2}\left\langle\tau_{D} e^{-\mathrm{i} \sigma_{3} \Theta} \sigma_{1} \sigma_{3} \Phi\right|=-\frac{q}{q^{\prime}}\left\langle\tau_{D} e^{-\mathrm{i} \sigma_{3} \Theta} \sigma_{3} \partial_{\omega} \Phi\right|-\frac{1}{2}\left\langle\tau_{D} e^{-\mathrm{i} \sigma_{3} \Theta} P_{c}\left(\mathcal{H}_{\omega}^{*}\right) \sigma_{3} \Phi\right| \\
& -\frac{1}{2}\left\langle\sigma_{3} \Phi \mid \xi_{j}\right\rangle\left\langle\tau_{D} e^{-\mathrm{i} \sigma_{3} \Theta} \sigma_{3} \xi_{j}\right|+\frac{1}{2}\left\langle\sigma_{3} \Phi \mid \xi_{j}\right\rangle\left\langle\tau_{D} e^{-\mathrm{i} \sigma_{3} \Theta} \sigma_{1} \sigma_{3} \xi_{j}\right| .
\end{aligned}
$$

By (2.19) we have

$$
\begin{aligned}
& -\frac{q}{q^{\prime}}\left\langle\tau_{D} e^{-\mathrm{i} \sigma_{3} \Theta} \sigma_{3} \partial_{\omega} \Phi\right|=\frac{q}{q^{\prime}}\left\langle R \mid \sigma_{3} \partial_{\omega}^{2} \Phi\right\rangle d \omega-\mathrm{i} \frac{q}{q^{\prime}}\left(q^{\prime}+\left\langle R \mid \partial_{\omega} \Phi\right\rangle\right) d \vartheta \\
& +\frac{q}{q^{\prime}}\left(\frac{\mathrm{i} v_{a}}{2} q^{\prime}+\left\langle\left(\partial_{a}+\frac{\mathrm{i}}{2} \sigma_{3} v_{a}\right) R \mid \sigma_{3} \partial_{\omega} \Phi\right\rangle\right) d D_{a}-\mathrm{i} \frac{q}{q^{\prime}}\left\langle x_{a} R, \partial_{\omega} \Phi\right\rangle d v_{a} .
\end{aligned}
$$

Set $\psi(U):=\frac{1}{2}\left\langle\sigma_{3} \Phi \mid R\right\rangle$. We have

$$
d \psi=\frac{1}{2}\left\langle\sigma_{3} \Phi \mid \partial_{\omega} R\right\rangle d \omega+\frac{1}{2}\left\langle\sigma_{3} \Phi \mid \xi_{j}\right\rangle\left(d z_{j}-d \bar{z}_{j}\right)+\frac{1}{2}\left\langle P_{c}^{*}(\omega) \sigma_{3} \Phi \mid f^{\prime}\right\rangle .
$$


We have $\nabla_{f} \psi=\frac{1}{2} P_{c}^{*}\left(\omega_{0}\right) P_{c}^{*}(\omega) \sigma_{3} \Phi$. We will use the notation $\partial_{j}:=\partial_{z_{j}}$ and $\partial_{\bar{j}}:=\partial_{\bar{z}_{j}}$. The sum of the last three terms on the rhs of (5.6) equals

$$
\begin{aligned}
& -\partial_{j} \psi d z_{j}-\partial_{\bar{j}} \psi d \bar{z}_{j}-\left\langle\nabla_{f} \psi \mid f^{\prime}\right\rangle-\frac{1}{2}\left\langle P_{N_{g}^{\perp}\left(\mathcal{H}_{\omega}^{*}\right)} \Phi \mid \sigma_{3} \partial_{\omega} R\right\rangle d \omega \\
& -\frac{\mathrm{i}}{2}\left\langle P_{N_{g}\left(\mathcal{H}_{\omega}\right)} \Phi \mid \sigma_{3} R\right\rangle d \vartheta-\frac{\mathrm{i}}{4}\left\langle P_{N_{g}\left(\mathcal{H}_{\omega}\right)} \Phi \mid x_{a} R\right\rangle d v_{a} \\
& +\frac{1}{2}\left\langle P_{N_{g}\left(\mathcal{H}_{\omega}\right)} \Phi \mid \sigma_{3}\left(\partial_{a}+\mathrm{i} \sigma_{3} \frac{v_{a}}{2}\right) R\right\rangle d D_{a} .
\end{aligned}
$$

Then we get

$$
\begin{aligned}
& \widetilde{B}=\frac{z_{j} d \bar{z}_{j}-\bar{z}_{j} d z_{j}}{2}+\frac{1}{2}\left\langle f \mid \sigma_{3} \sigma_{1} P_{c}(\omega) f^{\prime}\right\rangle-\partial_{j} \psi d z_{j} \\
& -\partial_{\bar{j}} \psi d \bar{z}_{j}-\left\langle\nabla_{f} \psi \mid f^{\prime}\right\rangle-\mathrm{i}\left(Q+\left\langle\frac{q}{q^{\prime}} \partial_{\omega} \Phi-\frac{1}{2} \sigma_{3} P_{N_{g}\left(\mathcal{H}_{\omega}\right)} \Phi \mid R\right\rangle\right) d \vartheta \\
& +\left(\frac{1}{2}\left\langle\sigma_{1} R \mid \sigma_{3} \partial_{\omega} R\right\rangle-\left\langle\frac{q}{q^{\prime}} \partial_{\omega} \Phi+\frac{1}{2} P_{N_{g}^{\perp}\left(\mathcal{H}_{\omega}^{*}\right)} \Phi \mid \sigma_{3} \partial_{\omega} R\right\rangle\right) d \omega \\
& +\left(\mathrm{i} \Pi_{a}+\left\langle\frac{q}{q^{\prime}} \partial_{\omega} \Phi-\frac{1}{2} P_{N_{g}\left(\mathcal{H}_{\omega}\right)} \Phi \mid \sigma_{3}\left(\partial_{a}+\mathrm{i} \sigma_{3} \frac{v_{a}}{2}\right) R\right\rangle\right) d D_{a} \\
& -\frac{\mathrm{i}}{2}\left(\frac{1}{2}\left\langle\sigma_{1} R \mid x_{a} R\right\rangle+\left\langle\frac{q}{q^{\prime}} \partial_{\omega} \Phi-\frac{1}{2} P_{N_{g}\left(\mathcal{H}_{\omega}\right)} \Phi \mid x_{a} R\right\rangle\right) d v_{a} .
\end{aligned}
$$

By (2.10) we have the following two equalities:

$$
\begin{aligned}
& \frac{1}{2} P_{N_{g}\left(\mathcal{H}_{\omega}\right)} \Phi=\frac{\langle\Phi \mid \Phi\rangle}{2 q^{\prime}} \partial_{\omega} \Phi=\frac{q}{q^{\prime}} \partial_{\omega} \Phi, \\
& \Phi=\frac{q}{q^{\prime}} \partial_{\omega} \Phi+\frac{1}{2} P_{N_{g}^{\perp}\left(\mathcal{H}_{\omega}^{*}\right)} \Phi .
\end{aligned}
$$

By (5.11) we have various cancelations in (5.10) yielding

$$
\begin{aligned}
& \widetilde{B}=\frac{z_{j} d \bar{z}_{j}-\bar{z}_{j} d z_{j}}{2}+\frac{1}{2}\left\langle f \mid \sigma_{3} \sigma_{1} P_{c}(\omega) f^{\prime}\right\rangle-d \psi-\mathrm{i} Q d \vartheta-\mathrm{i} \Pi_{a} d D_{a} \\
& +\frac{1}{2}\left\langle\sigma_{1} \sigma_{3} R \mid \partial_{\omega} R\right\rangle d \omega-\frac{\mathrm{i}}{4}\left\langle\sigma_{1} R \mid x_{a} R\right\rangle d v_{a} .
\end{aligned}
$$

We have $\Omega=d(\widetilde{B}+d \psi)=d \mathrm{~B}$ if we define

$$
\begin{aligned}
& \mathrm{B}:=\mathrm{i} \vartheta d Q+\mathrm{i} D_{a} d \Pi_{a}-\frac{\bar{z}_{j} d z_{j}-z_{j} d \bar{z}_{j}}{2}+\frac{1}{2}\left\langle f \mid \sigma_{3} \sigma_{1} P_{c}(\omega) f^{\prime}\right\rangle \\
& +\frac{1}{2}\left(\left\langle\sigma_{1} \sigma_{3} R \mid \partial_{\omega} R\right\rangle+\mathrm{i} v_{a}\left\langle\sigma_{1} R \mid x_{a} \partial_{\omega} R\right\rangle\right) d \omega \\
& +\frac{\mathrm{i}}{2} v_{a}\left\langle x_{a} \sigma_{1} R \mid \xi_{j}\right\rangle d z_{j}+\frac{\mathrm{i}}{2} v_{a}\left\langle x_{a} R \mid \xi_{j}\right\rangle d z_{j}+\frac{\mathrm{i}}{2} v_{a}\left\langle x_{a} \sigma_{1} R \mid P_{c}(\omega) f^{\prime}\right\rangle .
\end{aligned}
$$

Finally, this B satisfies (5.2)-(5.3) if we consider the formula

$$
\left(q^{\prime}+\partial_{\omega} Q(R)\right) d \omega=d Q-\partial_{j} Q(R) d z_{j}-\partial_{\bar{j}} Q(R) d \bar{z}_{j}-\left\langle\nabla_{f} Q(R) \mid f^{\prime}\right\rangle,
$$

where $\nabla_{f} Q(R)=P_{c}^{*}\left(\omega_{0}\right) P_{c}^{*}(\omega) \sigma_{1} R$. 
In general, given a function $F$, the following formula defines $\nabla_{f} F$ :

$$
\begin{aligned}
& d F=\partial_{Q} F d Q+\partial_{\Pi_{a}} F d \Pi_{a}+\partial_{\vartheta} F d Q+\partial_{D_{a}} F d D_{a} \\
& +\partial_{j} F d z_{j}+\partial_{\bar{j}} F d z_{\bar{j}}+\left\langle\nabla_{f} F \mid f^{\prime}\right\rangle,
\end{aligned}
$$

where $P_{c}^{*}\left(\omega_{0}\right) \nabla_{f} F=\nabla_{f} F$. We have the following result.

Lemma 5.2. Let us denote by $\mathcal{U}_{\Sigma}$ the subset of $\Sigma$ defined by the inequalities $|z| \leq \varepsilon_{0}$, $\|f\|_{H^{-K,-S}} \leq \varepsilon_{0},|\varrho(f)| \leq \varepsilon_{0}$ (here recall $\left.\varrho(f):=(Q(f), \Pi(f))\right),\left|\omega-\omega_{0}\right| \leq \varepsilon_{0}$ and $|v| \leq \varepsilon_{0}$. Then there exists a number $\varepsilon_{0}>0$ such that there exists a unique vectorfield $\mathcal{X}^{t}: U_{\Sigma} \rightarrow L^{2}$ which solves $i_{\mathcal{X}^{t}} \Omega_{t}=-\Gamma$, where $\Omega_{t}:=\Omega_{0}+t\left(\Omega-\Omega_{0}\right)$.

Remark 5.3. In Cu1 the existence of $\mathcal{X}^{t}$ is elementary and standard, due to the fact that $\Omega_{0}$ and $\Omega$ are differential forms in $L^{2}$ very close to each other. This is not true in our setting, where $\Omega_{0}: \Sigma \rightarrow B^{2}\left(L^{2}, \mathbb{C}\right)$ and where we cannot assume that $\Omega_{0}$ and $\Omega$ are close in $\Sigma$. This is for the simple reason that while the spaces $\Sigma_{n}$ are invariant for our NLS (1.1) (see Lemma 7.1 below), for $n>0$ the $\Sigma_{n}$ norms of the solutions grow in time. One of the main differences between [Cu1] and this paper lies here.

Proof. The proof ends after Lemma 5.7. We are considering $i_{\mathcal{X}^{t}} \Omega_{0}+t i_{\mathcal{X}^{t}} d \Gamma=-\Gamma$, where

$$
\begin{aligned}
& i_{X} \Omega_{0}=\mathrm{i}(X)_{\vartheta} d Q+\mathrm{i}(X)_{D_{a}} d \Pi_{a}-\mathrm{i}(X)_{Q} d \vartheta-\mathrm{i}(X)_{\Pi_{a}} d D_{a} \\
& -(X)_{\bar{j}} d z_{j}+(X)_{j} d z_{\bar{j}}+\left\langle\sigma_{1} \sigma_{3}(X)_{f} \mid f^{\prime}\right\rangle
\end{aligned}
$$

with $(X)_{\vartheta}$ the $\vartheta$-th component of $X$, etc. Set

$$
\begin{aligned}
\gamma_{1 a}: & =\left\langle x_{a} \sigma_{1} R \mid \xi_{j}\right\rangle d z_{j}+\left\langle x_{a} R \mid \xi_{j}\right\rangle d \bar{z}_{j}+\left\langle P_{c}^{*}(\omega) \sigma_{1} x_{a} R \mid f^{\prime}\right\rangle, \\
W: & =\varphi d Q-\varphi \partial_{j} Q(R) d z_{j}-\varphi \partial_{\bar{j}} Q(R) d \bar{z}_{j} \\
& -\varphi\left\langle P_{c}^{*}(\omega) \sigma_{1} R \mid f^{\prime}\right\rangle-2^{-1}\left\langle\sigma_{1} \sigma_{3} P_{d}(\omega) f \mid f^{\prime}\right\rangle .
\end{aligned}
$$

We have $\Gamma=\frac{\mathrm{i}}{2} v_{a} \gamma_{1 a}+W$ and

$$
d \Gamma=\frac{\mathrm{i}}{2} d v_{a} \wedge \gamma_{1 a}+\frac{\mathrm{i}}{2} v_{a} d \gamma_{1 a}+d W .
$$

Lemma 5.4. Using definitions (5.21)-(5.24) below, we have:

$$
\begin{gathered}
\gamma_{1 a}=\left\langle P_{c}^{*}(\omega) \sigma_{1} x_{a} f \mid f^{\prime}\right\rangle+\widetilde{\gamma}_{1 a}, \\
d v_{a}=-\frac{2}{Q} d \Pi_{a}+2\left[\left(\Pi_{a}-\Pi_{a}(R)\right) Q^{-2}+\frac{Q^{-1} \partial_{\omega} \Pi_{a}(R)}{q^{\prime}+\partial_{\omega} Q(R)}\right] d Q \\
+2 Q^{-1}\left\langle\mathrm{i} P_{c}^{*}(\omega) \sigma_{1} \sigma_{3} \partial_{a} f-\frac{\partial_{\omega} \Pi_{a}(R) P_{c}^{*}(\omega) \sigma_{1} f}{q^{\prime}+\partial_{\omega} Q(R)} \mid f^{\prime}\right\rangle+\widetilde{d v_{a}}, \\
d \gamma_{1 a}=\widehat{\gamma}_{1 a} \wedge\left(d Q-\left\langle P_{c}^{*}(\omega) \sigma_{1} f \mid f^{\prime}\right\rangle\right)+\widetilde{d \gamma_{1 a}} .
\end{gathered}
$$


In the above formulas we have

$$
\begin{aligned}
& \widetilde{\gamma}_{1 a}:=\left\langle x_{a} \sigma_{1} R \mid \xi_{j}\right\rangle d z_{j}+\left\langle x_{a} R \mid \xi_{j}\right\rangle d \bar{z}_{j}+\left\langle P_{c}^{*}(\omega) x_{a}\left(z \cdot \sigma_{1} \xi+\bar{z} \cdot \xi\right) \mid f^{\prime}\right\rangle \\
& \frac{Q}{2} \widetilde{d v_{a}}=\left(Q^{-1} \partial_{j} \Pi_{a}(R)-\frac{\partial_{\omega} \Pi_{a}(R) \partial_{j} Q(R)}{q^{\prime}+\partial_{\omega} Q(R)}\right) d z^{j} \\
& +\left(Q^{-1} \partial_{\bar{j}} \Pi_{a}(R)-\frac{\partial_{\omega} \Pi_{a}(R) \partial_{\bar{j}} Q(R)}{q^{\prime}+\partial_{\omega} Q(R)}\right) d \bar{z}^{j} \\
& \quad-\left\langle i P_{c}^{*}(\omega) \sigma_{3} \partial_{a}\left(z \cdot \sigma_{1} \xi+\bar{z} \cdot \xi\right)+\frac{\partial_{\omega} \Pi_{a}(R) P_{c}^{*}(\omega)\left(z \cdot \sigma_{1} \xi+\bar{z} \cdot \xi\right)}{q^{\prime}+\partial_{\omega} Q(R)} \mid f^{\prime}\right\rangle \\
& \widehat{\gamma}_{1 a}:=\frac{\partial_{\omega}\left\langle x_{a} \sigma_{1} R \mid \xi_{j}\right\rangle d z_{j}+\partial_{\omega}\left\langle x_{a} R \mid \xi_{j}\right\rangle d \bar{z}_{j}-\left\langle\partial_{\omega} P_{d}^{*}(\omega) \sigma_{1} x_{a} R \mid f^{\prime}\right\rangle}{q^{\prime}+\partial_{\omega} Q(R)} \\
& \widetilde{d \gamma}_{1 a}=\left(\partial_{k} Q(R) d z_{k}+\partial_{\bar{k}} Q(R) d \bar{z}_{k}+\left\langle P_{c}^{*}(\omega)\left(z \cdot \sigma_{1} \xi+\bar{z} \cdot \xi\right) \mid f^{\prime}\right\rangle\right) \wedge \widehat{\gamma}_{1 a} .
\end{aligned}
$$

Proof. By (4.5) we get

$$
\begin{aligned}
& d v_{a}=-\frac{2}{Q} d \Pi_{a}+2\left(\Pi_{a}-\Pi_{a}(R)\right) Q^{-2} d Q+2 Q^{-1} \partial_{j} \Pi_{a}(R) d z^{j} \\
& +2 Q^{-1} \partial_{\bar{j}} \Pi_{a}(R) d \bar{z}^{j}+2 Q^{-1}\left\langle\nabla_{f} \Pi_{a}(R) \mid f^{\prime}\right\rangle+2 Q^{-1} \partial_{\omega} \Pi_{a}(R) d \omega,
\end{aligned}
$$

where $\nabla_{f} \Pi_{a}(R)=\mathrm{i} P_{c}^{*}\left(\omega_{0}\right) P_{c}^{*}(\omega) \sigma_{1} \sigma_{3} \partial_{a} R$. Then by (5.14) we get

$$
\begin{aligned}
& d v_{a}=-\frac{2}{Q} d \Pi_{a}+2\left[\left(\Pi_{a}-\Pi_{a}(R)\right) Q^{-2}+\frac{Q^{-1} \partial_{\omega} \Pi_{a}(R)}{q^{\prime}+\partial_{\omega} Q(R)}\right] d Q \\
& +2 Q^{-1}\left[Q^{-1} \partial_{j} \Pi_{a}(R)-\frac{\partial_{\omega} \Pi_{a}(R) \partial_{j} Q(R)}{q^{\prime}+\partial_{\omega} Q(R)}\right] d z^{j} \\
& +2 Q^{-1}\left[Q^{-1} \partial_{\bar{j}} \Pi_{a}(R)-\frac{\partial_{\omega} \Pi_{a}(R) \partial_{j} Q(R)}{q^{\prime}+\partial_{\omega} Q(R)}\right] d \bar{z}^{j} \\
& +2 Q^{-1}\left\langle\mathrm{i} P_{c}^{*}(\omega) \sigma_{1} \sigma_{3} \partial_{a} R-\frac{\partial_{\omega} \Pi_{a}(R) P_{c}^{*}(\omega) \sigma_{1} R}{q^{\prime}+\partial_{\omega} Q(R)} \mid f^{\prime}\right\rangle .
\end{aligned}
$$

In particular we get (5.19) and (5.22). (5.21) is immediate from (5.16). (5.23)(5.24) follow by (5.14) from

$$
\begin{aligned}
& d \gamma_{1 a}=\left(\partial_{\omega}\left\langle x_{a} \sigma_{1} R \mid \xi_{j}\right\rangle d z_{j}+\partial_{\omega}\left\langle x_{a} R \mid \xi_{j}\right\rangle d \bar{z}_{j}-\left\langle\partial_{\omega} P_{d}^{*}(\omega) \sigma_{1} x_{a} R \mid f^{\prime}\right\rangle\right) \\
& \wedge \frac{d Q-\partial_{k} Q(R) d z_{k}-\partial_{\bar{k}} Q(R) d \bar{z}_{k}-\left\langle P_{c}^{*}(\omega) \sigma_{1} R \mid f^{\prime}\right\rangle}{q^{\prime}+\partial_{\omega} Q(R)} \\
& =\widehat{\gamma}_{1 a} \wedge\left(\left(d Q-\left\langle P_{c}^{*}(\omega) \sigma_{1} R \mid f^{\prime}\right\rangle\right)+\left(\partial_{k} Q(R) d z_{k}-\partial_{\bar{k}} Q(R) d \bar{z}_{k}\right)\right) .
\end{aligned}
$$

Set $H_{c}^{K, S}=P_{c}\left(\omega_{0}\right) H^{K, S}$ and denote

$$
\mathcal{P}^{K, S}=\mathbb{R}^{6} \times \mathbb{C}^{m} \times H_{c}^{K, S}, \quad \mathcal{P}=\mathcal{P}^{0,0} .
$$

The following lemma is straightforward.

Lemma 5.5. For $\varpi_{1}=\widetilde{d v_{a}}, \widetilde{\gamma}_{1 a}, \widehat{\gamma}_{1 a}$, we have $\varpi_{1} \in C^{\infty}\left(\mathcal{U}_{\Sigma}, B\left(\mathcal{P}^{-K,-S}, \mathbb{C}\right)\right)$ for fixed $C$

$$
\left\|\varpi_{1}\right\|_{B\left(\mathcal{P}^{-K,-S}, \mathbb{C}\right)} \leq C\left(|z|+\|f\|_{H^{-K,-S}}\right) .
$$


For $\varpi_{2}=\widetilde{d \gamma}_{1 a}$ we have $\varpi_{2} \in C^{\infty}\left(\mathcal{U}_{\Sigma}, B^{2}\left(\mathcal{P}^{-K,-S}, \mathbb{C}\right)\right)$ for fixed $C$

$$
\left\|\varpi_{2}\right\|_{B^{2}\left(\mathcal{P}^{-K,-S}, \mathbb{C}\right)} \leq C\left(|z|+\|f\|_{H^{-K,-S}}\right)^{2} .
$$

Furthermore, consider the contraction operator $X \rightarrow i_{X} \varpi_{2}$ and define $Y(X)$ by $i_{Y(X)} \Omega_{0}=i_{X} \varpi_{2}$. Then we have an equality of the form, summing on repeated index, with finite sums,

$$
\begin{aligned}
& (Y(X))_{\underline{j}}=R_{k}^{(j)}(X) \widetilde{\lambda}_{k}^{(j)} \text { for } \underline{j}=j, \bar{j}, \\
& (Y(X))_{f}=R_{k}(X) \Lambda_{k},
\end{aligned}
$$

with $R_{k}^{(j)}, R_{k} \in C^{\infty}\left(\mathcal{U}_{\Sigma}, B\left(\mathcal{P}^{-K,-S}, \mathbb{C}\right)\right)$ satisfying

$$
\left\|R_{k}^{(j)}\right\|_{B\left(\mathcal{P}^{-K,-S}, \mathbb{C}\right)}+\left\|R_{k}\right\|_{B\left(\mathcal{P}^{-K,-S}, \mathbb{C}\right)} \leq C\left(|z|+\|f\|_{H^{-K,-S}}\right),
$$

with $\widetilde{\lambda}_{k}^{(j)} \in C^{\infty}\left(\mathcal{U}_{\Sigma}, \mathbb{C}\right)$ and $\Lambda_{k} \in C^{\infty}\left(\mathcal{U}_{\Sigma}, H^{K, S}\right)$ satisfying estimates

$$
\left|\widetilde{\lambda}_{k}^{(j)}\right|+\left\|\Lambda_{k}\right\|_{H^{K, S}} \leq C\left(|z|+\|f\|_{H^{-K,-S}}\right) .
$$

For $E=\varpi_{1}, \varpi_{2}, R_{k}^{(\underline{j})}, R_{k}, \widetilde{\lambda}_{k}^{(\underline{j})}$ and $\Lambda_{k}$, we have $E=E(Q, \Pi, z, f, \varrho(f))$, where $E(Q, \Pi, z, f, \varrho)$ is smooth w.r.t. $(Q, \Pi)$ and $\varrho \in \mathbb{R}^{4}, z \in \mathbb{C}^{m}$ and $f \in H^{-K,-S}$.

Lemma 5.6. Set

$$
\begin{gathered}
G_{1}:=\frac{1}{2}\left\langle\sigma_{1} \sigma_{3} R \mid \partial_{\omega} R\right\rangle, \quad G_{2 a}:=\frac{\mathrm{i}}{2}\left\langle x_{a} \sigma_{1} R \mid \partial_{\omega} R\right\rangle \\
\widehat{W}:=\left(\partial_{j} G_{1}+v_{a} \partial_{j} G_{2 a}\right) d z_{j}+\left(\partial_{\bar{j}} G_{1}+v_{a} \partial_{\bar{j}} G_{2 a}\right) d \bar{z}_{j} \\
+\left\langle\nabla_{f} G_{1}+v_{a} \nabla_{f} G_{2 a}+2^{-1} \sigma_{1} \sigma_{3} \partial_{\omega} P_{d}(\omega) f \mid f^{\prime}\right\rangle+G_{2 a} \widetilde{d v_{a}} \\
\widetilde{d W}:=-\widehat{W} \wedge \frac{\partial_{j} Q(R) d z_{j}+\partial_{\bar{j}} Q(R) d \bar{z}_{j}+\left\langle P_{c}^{*}(\omega)\left(z \cdot \sigma_{1} \xi+\bar{z} \cdot \xi\right) \mid f^{\prime}\right\rangle}{q^{\prime}+\partial_{\omega} Q(R)} .
\end{gathered}
$$

Then we have

$$
\begin{aligned}
& d W=\widehat{W} \wedge \frac{d Q-\left\langle P_{c}^{*}(\omega) \sigma_{1} f \mid f^{\prime}\right\rangle}{q^{\prime}+\partial_{\omega} Q(R)}+\widetilde{d W} \\
& +G_{2 a}\left(d v_{a}-\widetilde{d v_{a}}\right) \wedge \frac{d Q-\partial_{j} Q(R) d z_{j}-\partial_{\bar{j}} Q(R) d \bar{z}_{j}-\left\langle P_{c}^{*}(\omega) \sigma_{1} R \mid f^{\prime}\right\rangle}{q^{\prime}+\partial_{\omega} Q(R)},
\end{aligned}
$$

where, for $\varpi_{1}=\widehat{W}$ and $\varpi_{2}=\widetilde{d W}$, the conclusions of Lemma 5.5 are satisfied.

Proof. Substituting (5.14) and using (5.3) we get

$$
W=\left(G_{1}+v_{a} G_{2 a}\right) d \omega-2^{-1}\left\langle\sigma_{1} \sigma_{3} P_{d}(\omega) f \mid f^{\prime}\right\rangle .
$$

By (5.14) we obtain the following formula, from which we get (5.31):

$$
\begin{aligned}
& d W=\left(d G_{1}+v_{a} d G_{2 a}+2^{-1}\left\langle\sigma_{1} \sigma_{3} \partial_{\omega} P_{d}(\omega) f \mid f^{\prime}\right\rangle+G_{2 a} d v_{a}\right) \wedge d \omega \\
& =\left(\widehat{W}+G_{2 a}\left(d v_{a}-\widetilde{d v_{a}}\right)\right) \wedge d \omega .
\end{aligned}
$$

Then substitute (5.14).

We reframe the equation for $\mathcal{X}^{t}$. 
Lemma 5.7. For $Y$ defined by $i_{Y} \Omega_{0}=-\Gamma$, then equation $i_{\mathcal{X}^{t}} \Omega_{t}=-\Gamma$ is equivalent to

$$
(1+t \mathcal{K}) \mathcal{X}^{t}=Y
$$

where the operator $\mathcal{K}$ satisfies the following properties:

(1) For the component $(\mathcal{K} X)_{f}$ the following facts hold:

$$
(\mathcal{K} X)_{f}=A_{a}(X) \partial_{a} f+\sigma_{3}\left(B_{a}(X) x_{a}+C(X)\right) f+D_{i}(X) \Psi_{i},
$$

where the last is a finite sum with $\Psi_{i} \in C^{\infty}\left(\mathcal{U}_{\Sigma}, H^{K, S}\right)$ with

$$
\left\|\Psi_{i}\right\|_{H^{K, S}} \leq C\left(|z|+\|f\|_{H^{-K,-S}}\right) .
$$

For $L=A_{a}, B_{a}, C, D_{i}$, we have $L \in C^{0}\left(\mathcal{U}_{\Sigma}, B(\mathcal{P}, \mathbb{C})\right.$ ) (see (5.25) ), with

$$
L(X)=\left\langle L_{1 b} \sigma_{1} \sigma_{3} \partial_{b} f+L_{2 b} \sigma_{1} x_{b} f+L_{3} \sigma_{1} f \mid(X)_{f}\right\rangle+\widetilde{L}(X)
$$

with $\widetilde{L} \in C^{\infty}\left(\mathcal{U}_{\Sigma}, B\left(\mathcal{P}^{-K,-S}, \mathbb{C}\right)\right),\|\widetilde{L}\|_{B\left(\mathcal{P}^{-K,-S}, \mathbb{C}\right)} \leq C\left(|z|+\|f\|_{H^{-K,-S}}\right)$ and where $L_{1 b}, L_{2 b}, L_{3}$ are in $C^{\infty}\left(\mathcal{U}_{\Sigma}, \mathbb{C}\right)$.

(2) The $z_{j}$-th component $(\mathcal{K} X)_{j}$ is of the form (5.36) with the estimates

$$
\left|L_{1 b}\right|+\left|L_{2 b}\right|+\left|L_{3}\right|+\|\widetilde{L}\|_{B\left(\mathcal{P}^{-K,-S}, \mathbb{C}\right)} \leq C\left(|z|+\|f\|_{H^{-K,-S}}\right) .
$$

(3) For $G$ any of the above $L_{1 b}, L_{2 b}, L_{3}, \widetilde{L}$, we have $G=G(Q, \Pi, z, f, \varrho(f))$, where $G(Q, \Pi, z, f, \varrho)$ is smooth w.r.t. $(Q, \Pi)$ and $\varrho \in \mathbb{R}^{4}, z \in \mathbb{C}^{m}$ and $f \in H^{-K,-S}$.

Proof. We have $i_{\frac{\partial}{\partial Q}} \Omega_{0}=-\mathrm{i} d \vartheta$ by (5.1) and $i_{\frac{\partial}{\partial Q}} \Omega=-\mathrm{i} d \vartheta$ by Lemmas 4.1 and 4.4 , Similarly $i_{\frac{\partial}{\partial \Pi_{a}}} \Omega_{0}=i_{\frac{\partial}{\partial \Pi_{a}}} \Omega=-\mathrm{i} d D_{a}$. So in particular $i_{\frac{\partial}{\partial Q}} d \Gamma=i_{\frac{\partial}{\partial D_{a}}} d \Gamma=0$. Then $(\Gamma)_{\vartheta}=(\Gamma)_{D_{a}}=0$ implies $\left(\mathcal{X}^{t}\right)_{Q}=\left(\mathcal{X}^{t}\right)_{\Pi_{a}}=0$. Then

$$
\begin{aligned}
& i_{\mathcal{X}^{t}} d \Gamma=\frac{\mathrm{i}}{2}\left(d v_{a}\left(\mathcal{X}^{t}\right) \gamma_{1 a}-\gamma_{1 a}\left(\mathcal{X}^{t}\right) d v_{a}+v_{a} i_{\mathcal{X}^{t}} d \gamma_{1 a}\right)+i_{\mathcal{X}^{t}} d W \\
& =\frac{\mathrm{i}}{2} d v_{a}\left(\mathcal{X}^{t}\right)\left(\left\langle P_{c}^{*}(\omega) \sigma_{1} x_{a} f \mid f^{\prime}\right\rangle+\widetilde{\gamma}_{1 a}\right)-\frac{\mathrm{i}}{2} \gamma_{1 a}\left(\mathcal{X}^{t}\right)\left(\widetilde{d v_{a}}+d v_{a}-\widetilde{d v_{a}}\right) \\
& +\frac{\mathrm{i}}{2} v_{a} \widehat{\gamma}_{1 a}\left(\mathcal{X}^{t}\right)\left(d Q-\left\langle P_{c}^{*}(\omega) \sigma_{1} f \mid f^{\prime}\right\rangle\right)+\frac{\mathrm{i}}{2} v_{a}\left\langle P_{c}^{*}(\omega) \sigma_{1} f \mid\left(\mathcal{X}^{t}\right)_{f}\right\rangle \widehat{\gamma}_{1 a} \\
& +\widehat{W}\left(\mathcal{X}^{t}\right) \frac{d Q-\left\langle P_{c}^{*}(\omega) \sigma_{1} f \mid f^{\prime}\right\rangle}{q^{\prime}+\partial_{\omega} Q(R)}+\frac{\left\langle P_{c}^{*}(\omega) \sigma_{1} f \mid\left(\mathcal{X}^{t}\right)_{f}\right\rangle}{q^{\prime}+\partial_{\omega} Q(R)}+i_{\mathcal{X}^{t}} \widetilde{d W} \\
& +G_{2 a}\left(d v_{a}\left(\mathcal{X}^{t}\right)-\widetilde{d v_{a}}\left(\mathcal{X}^{t}\right)\right) \frac{d Q-\partial_{j} Q(R) d z_{j}-\partial_{\bar{j}} Q(R) d \bar{z}_{j}-\left\langle P_{c}^{*}(\omega) \sigma_{1} R \mid f^{\prime}\right\rangle}{q^{\prime}+\partial_{\omega} Q(R)} \\
& +G_{2 a} \frac{\partial_{j} Q(R)\left(\mathcal{X}^{t}\right)_{j}+\partial_{\bar{j}} Q(R)\left(\mathcal{X}^{t}\right)_{\bar{j}}+\left\langle P_{c}^{*}(\omega) \sigma_{1} R \mid\left(\mathcal{X}^{t}\right)_{f}\right\rangle}{q^{\prime}+\partial_{\omega} Q(R)}\left(d v_{a}-\widetilde{d v_{a}}\right) .
\end{aligned}
$$


So for $\widetilde{Q}$ and $\widetilde{\Pi}_{a}$ two 1 -forms irrelevant in the sequel (since we are not interested in $\left(\mathcal{X}^{t}\right)_{\vartheta}$ and $\left(\mathcal{X}^{t}\right)_{D_{a}}$ ), after a tedious but elementary computation we have

$$
\begin{aligned}
& i_{\mathcal{X}^{t}} d \Gamma=\widetilde{Q}\left(\mathcal{X}^{t}\right) d Q+\widetilde{\Pi}_{a}\left(\mathcal{X}^{t}\right) d \Pi_{a}+\frac{\mathrm{i}}{2} d v_{a}\left(\mathcal{X}^{t}\right) \widetilde{\gamma}_{1 a}-\frac{\mathrm{i}}{2} \gamma_{1 a}\left(\mathcal{X}^{t}\right) \widetilde{d v_{a}} \\
& +\frac{\mathrm{i}}{2} v_{a} i_{\mathcal{X}^{t}} \widetilde{d \gamma_{1 a}}+i_{\mathcal{X}^{t}} \widetilde{d W}+\left\langle P_{c}^{*}(\omega) \sigma_{1} f \mid\left(\mathcal{X}^{t}\right)_{f}\right\rangle\left(\frac{\mathrm{i}}{2} v_{a} \widehat{\gamma}_{1 a}+\frac{\widetilde{W}}{q^{\prime}+\partial_{\omega} Q(R)}\right) \\
& -\left(q^{\prime}+\partial_{\omega} Q(R)\right)^{-1} G_{2 a}\left(d v_{a}\left(\mathcal{X}^{t}\right)-\widetilde{d v_{a}}\left(\mathcal{X}^{t}\right)\right)\left(\partial_{j} Q(R) d z_{j}+\partial_{j} Q(R) d \bar{z}_{j}\right) \\
& +\widehat{\Gamma}_{3}\left(\mathcal{X}^{t}\right)\left\langle P_{c}^{*}(\omega) \sigma_{1} f \mid f^{\prime}\right\rangle+\widehat{\Gamma}_{2 a}\left(\mathcal{X}^{t}\right)\left\langle P_{c}^{*}(\omega) \sigma_{1} x_{a} f \mid f^{\prime}\right\rangle \\
& +\widehat{\Gamma}_{1 a}\left(\mathcal{X}^{t}\right)\left\langle P_{c}^{*}(\omega) \sigma_{1} \sigma_{3} \partial_{a} f \mid f^{\prime}\right\rangle,
\end{aligned}
$$

with (by (5.19))

$$
\begin{aligned}
& \widehat{\Gamma}_{3}:=\frac{-\widehat{W}}{q^{\prime}+\partial_{\omega} Q(R)}-\frac{\mathrm{i}}{2} v_{a} \widehat{\gamma}_{1 a}+\mathrm{i} Q^{-1} \frac{\partial_{\omega} \Pi_{a}(R)}{q^{\prime}+\partial_{\omega} Q(R)} \gamma_{1 a} \\
& -\frac{2 G_{2 a}}{Q\left(q^{\prime}+\partial_{\omega}(R)\right)}\left\langle\mathrm{i} P_{c}^{*}(\omega) \sigma_{1} \sigma_{3} \partial_{a} f-\frac{\partial_{\omega} \Pi_{a}(R) P_{c}^{*}(\omega) \sigma_{1} f}{q^{\prime}+\partial_{\omega} Q(R)} \mid f^{\prime}\right\rangle \\
& -2 G_{2 a} \partial_{\omega} \Pi_{a}(R) \frac{\partial_{k} Q(R) d z_{k}+\partial_{\bar{k}} Q(R) d \bar{z}_{k}+\left\langle P_{c}^{*}(\omega) \sigma_{1} R \mid f^{\prime}\right\rangle}{Q\left(q^{\prime}+\partial_{\omega} Q(R)\right)^{2}} \\
& \widehat{\Gamma}_{2 a}:=\frac{\mathrm{i}}{2} d v_{a}, \\
& \widehat{\Gamma}_{1 a}:=\frac{\gamma_{1 a}}{Q}+2 G_{2 a} \frac{\partial_{k} Q(R) d z_{k}+\partial_{\bar{k}} Q(R) d \bar{z}_{k}+\left\langle P_{c}^{*}(\omega) \sigma_{1} R \mid f^{\prime}\right\rangle}{Q\left(q^{\prime}+\partial_{\omega} Q(R)\right)} .
\end{aligned}
$$

The operator $\mathcal{K}$ is defined by $i_{\mathcal{K} X} \Omega_{0}=i_{X} d \Gamma$ for any $X$. By (5.15) this implies that (5.40)

$$
\begin{aligned}
& (\mathcal{K} X)_{f}=\widehat{\Gamma}_{3}(X) P_{c}\left(\omega_{0}\right) P_{c}(\omega) \sigma_{3} f+\widehat{\Gamma}_{2 a}(X) P_{c}\left(\omega_{0}\right) P_{c}(\omega) \sigma_{3} x_{a} f+(\widehat{\mathcal{R}}(X))_{f} \\
& +\widehat{\Gamma}_{1 a}(X) P_{c}\left(\omega_{0}\right) P_{c}(\omega) \partial_{a} f+\left\langle P_{c}^{*}(\omega) \sigma_{1} f \mid(X)_{f}\right\rangle \sigma_{3} \sigma_{1}\left(\frac{\mathrm{i}}{2} v_{a}\left(\widehat{\gamma}_{1 a}\right)_{f}+(\widehat{W})_{f}\right)
\end{aligned}
$$

where $i_{\widehat{\mathcal{R}}(X)} \Omega_{0}:=\frac{\mathrm{i}}{2} v_{a} i_{X} \widetilde{d \gamma_{1 a}}+i_{X} \widetilde{d W}$ and where $\left(\widehat{\gamma}_{1 a}\right)_{f}$, resp. $(\widehat{W})_{f}$, are the analogues of $(\Gamma)_{f}$ of the expansion of $\Gamma$ under (5.2). They are like the $\Psi_{i}$ of the statement by Lemma 5.5. resp. 5.6. By Lemma 5.5 we have $(\widehat{\mathcal{R}}(X))_{f}=R_{k}(X) \Lambda_{k}$, a sum of the form (5.28) which satisfies (5.29) - (5.30). Claim (1) follows from (5.40) after expansions like $P_{c}\left(\omega_{0}\right) P_{c}(\omega) \sigma_{3} f=\sigma_{3} f+\left(1-P_{c}\left(\omega_{0}\right) P_{c}(\omega)\right) \sigma_{3} f$, where the second term on the right is like a $\Psi_{i}$.

The terms in (5.38) contributing to $(\mathcal{K} X)_{j}$ are the last two in the first line and those in the second and third lines. Then claim (2) follows from Lemma 5.5. Claim (3) follows from Lemmas 5.1 and 5.5 .

We can apply the Fredholm alternative to prove existence and uniqueness of a solution to (5.33). We check that $(1+t \mathcal{T}) X=0$ admits no solution, with $\mathcal{T}$ the adjoint of $\mathcal{K}$ with respect to $\Omega_{0} . \mathcal{T}$ is like $\mathcal{K}$ and satisfies (5.34)- We show that $X=-t \mathcal{T} X$ does not have nontrivial solutions for $|t| \leq 3$ for the $\varepsilon_{0}$ (see the statement of Lemma [5.2), sufficiently small. We prove this by showing that there 
is a fixed constant $\kappa$ such that the following hold:

$$
\begin{aligned}
& \left(\mathcal{T}^{n} X\right)_{f}=A_{a}^{(n)}(X) \partial_{a} f+\sigma_{3}\left[B_{a}^{(n)}(X) x_{a}+C^{(n)}(X)\right] f+D_{i}^{(n)}(X) \Psi_{i}, \\
& \left(\mathcal{T}^{n} X\right)_{\underline{j}}=Z_{\underline{j}}^{(n)}(X), \\
& \left|L^{(n)}(X)\right| \leq \kappa^{n-1}\left(|z|+\|f\|_{H^{-K,-S}}+|\varrho(f)|\right)^{n-1}|L(X)|,
\end{aligned}
$$

for $L^{(n)}=A_{a}^{(n)}, B_{a}^{(n)}, C^{(n)}, D_{1}^{(n)}, Z_{j}^{(n)}$. This will imply that the solutions of $X=-t \mathcal{T} X$ are trivial for $\kappa \varepsilon_{0}$ sufficiently small. We have

$$
\begin{aligned}
& L^{(n+1)}(X)=\widetilde{L}\left(\mathcal{K}^{n} X\right) \\
& +\left\langle L_{1 b} \sigma_{1} \sigma_{3} \partial_{b} f+L_{2 b} \sigma_{1} x_{b} f+L_{3} \sigma_{1} f \mid\left(\mathcal{T}^{n} X\right)_{f}\right\rangle .
\end{aligned}
$$

We have $\left|\widetilde{L}\left(\mathcal{K}^{n} X\right)\right| \leq c_{0} \kappa^{n-1}\left(|z|+\|f\|_{H^{-K,-S}}+|\varrho(f)|\right)^{n}|L(X)|$ for a fixed constant $c_{0}=c_{0}(\widetilde{L})$, by induction and Lemma 5.7. Substituting (5.41), the second line in (5.42) becomes

$$
\begin{aligned}
& \left\langle L_{1 b} \sigma_{1} \sigma_{3} \partial_{b} f+L_{2 b} \sigma_{1} x_{b} f+L_{3} \sigma_{1} f \mid A_{a}^{(n)}(X) \partial_{a} f+\cdots+D_{i}^{(n)}(X) \Psi_{i}\right\rangle \\
= & \delta_{a b}\left(L_{1 b} B_{a}^{(n)}(X)-L_{2 b} A_{a}^{(n)}(X)\right) Q(f) \\
+ & D_{i}^{(n)}(X)\left\langle L_{1 b} \sigma_{1} \sigma_{3} \partial_{b} f+L_{2 b} \sigma_{1} x_{b} f+L_{3} \sigma_{1} f \mid \Psi_{i}\right\rangle,
\end{aligned}
$$

where we used

$$
\begin{aligned}
& \left\langle\sigma_{1} \sigma_{3} \partial_{a} f \mid \partial_{b} f\right\rangle=\left\langle\sigma_{1} \partial_{a} f \mid f\right\rangle=\left\langle\sigma_{1} \sigma_{3} x_{a} f \mid x_{b} f\right\rangle=\left\langle\sigma_{1} \sigma_{3} x_{a} f \mid f\right\rangle \\
& =\left\langle\sigma_{1} \sigma_{3} f \mid f\right\rangle=0 \text { and } 2\left\langle\sigma_{1} \partial_{a} f \mid x_{b} f\right\rangle=-\delta_{a b}\|f\|_{L^{2}}^{2}
\end{aligned}
$$

The absolute value of the rhs of (5.43) is by induction

$$
\leq c_{1} \kappa^{n-1}\left(|z|+\|f\|_{H^{-K,-S}}+|\varrho(f)|\right)^{n}|L(X)|
$$

for a fixed constant $c_{1}=c_{1}(L)$. So

$$
\left|L^{(n+1)}(X)\right| \leq \kappa^{n+1}\left(|z|+\|f\|_{H^{-K,-S}}+|\varrho(f)|\right)^{n}|L(X)|
$$

if the constant $\kappa$ is chosen sufficiently large. The proof of Lemma 5.2 is concluded. The fact that $|L(X)| \leq c_{3}\left(|z|+\|f\|_{\Sigma}\right)\|X\|_{L^{2}}$ does not need to be used.

The following is another important step in the proof. We need to show that the flow of $\mathcal{X}^{t}$ corresponds to the flow of a quasilinear hyperbolic symmetric system. To study this system, wellposedness and regularity with respect to the initial data, we need more information on $\mathcal{X}^{t}$. We remark that $H^{K, S}$ has been fixed with any preassigned pair $(K, S)$. We will take both $K$ and $S$ very large.

Lemma 5.8. For $\mathcal{X}^{t}$ the vectorfield of Lemma [5.2, we have

$$
\begin{aligned}
& \left(\mathcal{X}^{t}\right)_{f}=\mathcal{L} f+\mathcal{D}, \quad\left(\mathcal{X}^{t}\right)_{\underline{j}}=Z_{\underline{j}} \text { for } \underline{j} \in\{j, \bar{j}\}, \\
& \mathcal{L}:=\mathcal{A}_{a} \partial_{a}+\left(\mathcal{B}_{a} x_{a}+\mathcal{C}\right) \sigma_{3},
\end{aligned}
$$

where the coefficients satisfy the following properties:

(1) $\mathcal{A}_{a}$ are real valued functions. $\mathcal{B}_{a}$ and $\mathcal{C}$ are imaginary valued. $Z_{j}$ has values in $\mathbb{C}$ with $Z_{\bar{j}}=\bar{Z}_{j}$. D has values in $H^{K, S}$.

(2) We have $G=G(t, z, f, \varrho(f))$ for $G=\mathcal{A}_{a}, \mathcal{B}_{a}, \mathcal{C}, \mathcal{D}, Z_{\bar{j}}$, for functions $G(t, z, f, \varrho)$ smooth in $t, z$, in $f \in H^{-K,-S}$ and in $\rho$. 
(3) We have

$$
\begin{aligned}
& \left|\mathcal{A}_{a}\right| \leq C(K, S)\left(|z|^{2}+\|f\|_{H^{-K,-S}}^{2}+|\varrho(f)|\right), \\
& |Z|+|\mathcal{C}|+\|\mathcal{D}\|_{H^{K, S}} \\
& \leq C(K, S)\left(|z|+\|f\|_{H^{-K,-S}}+|\varrho(f)|\right)\left(|z|+\|f\|_{H^{-K,-S}}\right) .
\end{aligned}
$$

(4) We have $\mathcal{B}_{a}=-\frac{\mathrm{i}}{2} v_{a}-\frac{\mathrm{i}}{2} t d v_{a}\left(\mathcal{X}^{t}\right)$.

Proof. Let us start by assuming that $\mathcal{X}^{t}$ is of the form (5.46). Let $X$ be a vector such that $\sigma_{1} X=\bar{X}$. Then $\Gamma(X)$ is imaginary. We have $\overline{\Omega_{t}\left(\mathcal{X}^{t}, X\right)}=-\Omega_{t}\left(\sigma_{1} \overline{\mathcal{X}^{t}}, X\right)$. For $t=1$ it is straightforward and for $t=0$ it can be checked using Lemmas 2.3 and 2.4. Since also $\overline{\Omega_{t}\left(\mathcal{X}^{t}, X\right)}=-\Gamma(X)=\Omega_{t}\left(\mathcal{X}^{t}, X\right)$, we get $\sigma_{1} \overline{\mathcal{X}^{t}}=\mathcal{X}^{t}$. From this discussion we can conclude that claim (1) holds if (5.46) is true.

Let $Y$ be defined by $i_{Y} \Omega_{0}=-\Gamma$. Then

$$
\begin{aligned}
& (Y)_{j}=-\Gamma_{\bar{j}}, \quad(Y)_{\bar{j}}=\Gamma_{j}, \\
& (Y)_{f}=\sigma_{3} \sigma_{1} \Gamma_{f}=-\frac{\mathrm{i}}{2} \sigma_{3} v_{a} x_{a} f+\varphi \sigma_{3} f+\widetilde{Y}_{f},
\end{aligned}
$$

with $(Y)_{j}$ and $\widetilde{Y}_{f}$, such as $G=G(t, z, f, \varrho(f))$ in the statement above, smooth in $z$, in $f \in H^{-K,-S}$ and in $\rho$ and s.t., by (5.3),

$$
\left|(Y)_{\underline{j}}\right|+\left\|\widetilde{Y}_{f}\right\|_{H^{K, S}} \leq C\left(|z|+\|f\|_{H^{-K,-S}}\right)\left(|z|+\|f\|_{H^{-K,-S}}+|\varrho(f)|\right) .
$$

Our first claim is that the following series converge:

$$
\begin{aligned}
& \left(\mathcal{X}^{t}\right)_{f}=\sum_{n=0}^{\infty}(-1)^{n} t^{n}\left(\mathcal{K}^{n} Y\right)_{f} \\
& =: \widehat{A}_{a}(Y) \partial_{a} f+\sigma_{3}\left(\widehat{B}_{a}(Y) x_{a}+\widehat{C}(Y)\right) f+\widehat{D}_{i}(Y) \Psi_{i}, \\
& \left(\mathcal{X}^{t}\right)_{\underline{j}}=\sum_{n=0}^{\infty}(-1)^{n} t^{n}\left(\mathcal{K}^{n} Y\right)_{\underline{j}}=: \widehat{Z}_{\underline{j}}(Y),
\end{aligned}
$$

with, for $L=A_{a}, B_{a}, C, D_{i}, Z_{\underline{j}}$,

$$
\widehat{L}(Y)=\sum_{n=0}^{\infty}(-1)^{n} t^{n} L^{(n)}(Y),
$$

for $L^{(n)}=A_{a}^{(n)}, B_{a}^{(n)}, C^{(n)}, D_{i}^{(n)}, Z_{j}^{(n)}$ defined as in (5.41) but with $\mathcal{K}$ instead of $\mathcal{T}$. To prove the first claim, notice that by the proof of Lemma 5.7 we can conclude that there exists a fixed constant $\kappa$ such that the following analogue of (5.45) holds:

$$
\begin{aligned}
& \left|L^{(n+1)}(Y)\right| \leq \kappa^{n+1}\left(|z|+\|f\|_{H^{-K,-S}}+|\varrho(f)|\right)^{n}|L(Y)| \\
& \leq \kappa^{n+1}\left(|z|+\|f\|_{H^{-K,-S}}+|\varrho(f)|\right)^{n}\left(|z|^{2}+\|f\|_{H^{-K,-S}}^{2}+|\varrho(f)|\right),
\end{aligned}
$$

where in the second inequality we exploited (5.48)-(5.49) and Lemma 5.7 which yield

$$
|L(Y)| \leq C_{L}\left(|z|^{2}+\|f\|_{H^{-K,-S}}^{2}+|\varrho(f)|\right) .
$$

In the case of $L=\mathcal{C}, Z, D_{i}$ we have a better estimate:

$$
|L(Y)| \leq C_{0}\left(|z|+\|f\|_{H^{-K,-S}}\right)\left(|z|+\|f\|_{H^{-K,-S}}+|\varrho(f)|\right) .
$$


This yields the first claim, and proves that $\mathcal{X}^{t}$ is of the form of (5.46) and that (5.47) holds. A second claim is that

$$
L^{(n+1)}(Y)=\sum_{i_{1} \in I_{1}} \varsigma_{i_{1}}(Y) \sum_{\sigma, \tau} \varepsilon_{i_{1}}(\sigma, \tau) \prod_{j=0}^{n} \ell_{\sigma(j)} \varpi_{\tau(j)},
$$

where we have the following:

(i) The sums on $\sigma$ and $\tau$ are over all maps $\sigma: \mathbb{Z}_{n} \rightarrow I_{2}$ and $\tau: \mathbb{Z}_{n} \rightarrow I_{3}$ with $I_{j}$, for $j=1,2,3$, the three finite sets described below. For $i_{1} \in I_{1}$ we have functions $(\sigma, \tau) \rightarrow \varepsilon_{i_{1}}(\sigma, \tau)$ with values in $\{0,1\}$.

(ii) $\varsigma_{i_{1}}(Y)$ varies in all possible ways among $\left\langle\sigma_{1} \sigma_{3} \partial_{a} f \mid Y\right\rangle,\left\langle\sigma_{1} x_{a} f \mid Y\right\rangle,\left\langle\sigma_{1} f \mid Y\right\rangle$ and $\widetilde{L}(Y)$, for $L=A_{a}, B_{a}, C, D_{i},(\mathcal{K})_{j}$. We denote by $I_{1}$ the set of these functions.

(iii) $\ell_{i_{2}}$ varies among $L_{1 b}, L_{2 b}, L_{3}$, for $L=A_{a}, B_{a}, C, D_{i},(\mathcal{K})_{j}$. We denote by $I_{2}$ the set of these functions.

(iv) $\varpi_{i_{3}}$ varies among $1,\|f\|_{2}^{2},\left\langle\sigma_{1} \sigma_{3} \partial_{a} f \mid \Psi_{i}\right\rangle,\left\langle\sigma_{1} x_{a} f \mid \Psi_{i}\right\rangle,\left\langle\sigma_{1} f \mid \Psi_{i}\right\rangle, \widetilde{L}\left(\Psi_{i}\right)$, $\widetilde{L}\left(\Psi_{i}\right), \widetilde{L}\left(\partial_{a} f\right), \widetilde{L}\left(\sigma_{3} x_{a} f\right), \widetilde{L}\left(\sigma_{3} f\right)$ for $L=A_{a}, B_{a}, C, D_{i},(\mathcal{K})_{j}$. We denote by $I_{3}$ the set of these functions.

The second claim is true for $n=0$ since by (5.36)

$$
L(Y)=L_{1 b}\left\langle\sigma_{1} \sigma_{3} \partial_{b} f \mid Y\right\rangle+L_{2 b}\left\langle\sigma_{1} x_{b} f \mid Y\right\rangle+L_{3}\left\langle\sigma_{1} f \mid Y\right\rangle+\widetilde{L}(Y) .
$$

In this case $\varpi_{i_{3}}=1$. Suppose that the second claim holds for $n-1$. For definiteness we will look at the case of $\mathcal{A}_{a}^{(n+1)}(Y)$. We have

$$
\begin{aligned}
& \mathcal{A}_{a}^{(n+1)}(Y)=\widetilde{\mathcal{A}_{a}}\left(\mathcal{K}^{n} Y\right)+\left(\mathcal{A}_{a}\right)_{1 b}\left\langle\sigma_{1} \sigma_{3} \partial_{b} f \mid\left(\mathcal{K}^{n} Y\right)_{f}\right\rangle \\
& +\left(\mathcal{A}_{a}\right)_{2 b}\left\langle\sigma_{1} x_{b} f \mid\left(\mathcal{K}^{n} Y\right)_{f}\right\rangle+\left(\mathcal{A}_{a}\right)_{3}\left\langle\sigma_{1} f \mid\left(\mathcal{K}^{n} Y\right)_{f}\right\rangle .
\end{aligned}
$$

For definiteness let us look at the last term on the first line. Then, substituting

$$
\left(\mathcal{K}^{n} Y\right)_{f}=A_{a}^{(n)}(Y) \partial_{a} f+\sigma_{3}\left[B_{a}^{(n)}(Y) x_{a}+C^{(n)}(Y)\right] f+D_{i}^{(n)}(Y) \Psi_{i}
$$

and using the induction hypothesis, we obtain the desired result. By proceeding in the same way in all the other cases we get the second claim.

What is left in the proof of Lemma 5.8 is the regularity in claim (2). The functions in (i)-(iv) are like the $G(t, z, f, \varrho)$ in the statement of claim (2). But then claim (2) follows by the elementary fact that, if $f_{0}, \ldots, f_{N}$ are smooth scalar functions, if $\sigma$ varies in the set $\mathfrak{F}\left(\mathbb{Z}_{n}, \mathbb{Z}_{N}\right)$ of all the maps $\mathbb{Z}_{n} \rightarrow \mathbb{Z}_{N}$ and we consider arbitrary maps $\varepsilon_{n}: \mathfrak{F}\left(\mathbb{Z}_{n}, \mathbb{Z}_{N}\right) \rightarrow\{0,1\}$, then there exists a fixed $\delta>0$ such that if $\left|f_{j}\right|_{\infty}<\delta$ for all $j$, then the following series converges to a smooth function:

$$
\sum_{n=0}^{\infty} \sum_{\sigma \in \mathfrak{F}\left(\mathbb{Z}_{n}, \mathbb{Z}_{N}\right)} \varepsilon_{n}(\sigma) \prod_{j=0}^{n} f_{\sigma(j)} .
$$

We sketch a proof assuming that the $f_{j}$ are functions of one scalar variable. It is enough to show that the series obtained differentiating term by term in (5.56) are totally convergent. This is immediate for the 0 -th derivative. The $k$-th derivative yields, for $|\mu|=\sum_{j}|\mu(j)|$, a series of the form

$$
\sum_{n=0}^{\infty} A_{n} \text { with } A_{n}=\sum_{\substack{\sigma \in \mathfrak{F}\left(\mathbb{Z}_{n}, \mathbb{Z}_{N}\right) \\ \mu \in \mathfrak{F}\left(\mathbb{Z}_{n}, \mathbb{Z}_{k}\right) \text { s.t. }|\mu|=k}} \varepsilon_{n}(\sigma) \prod_{j=0}^{n} f_{\sigma(j)}^{(\mu(j))} .
$$


Then we have the bound

$$
\begin{aligned}
& \left|A_{n}\right| \leq(N+1)^{n+1}(n+1)^{k} \sup \left\{\left|\prod_{j=0}^{n} f_{\sigma(j)}^{(\mu(j))}\right| \text { s.t. }(\sigma, \mu) \text { as in (5.57) }\right\} \\
& \leq(N+1)^{n+1}(n+1)^{k} \delta^{n-k} \sup _{j}\left\|f_{j}\right\|_{W^{k, \infty}}^{k} .
\end{aligned}
$$

So $\left|A_{n}\right| \leq \delta^{n+1-k}(N+1)^{n+1}(n+1)^{k} C_{k}^{k}$ for $C_{k}=\sup _{j}\left\|f_{j}\right\|_{W^{k, \infty}}$. Then for $(N+$ 1) $\delta_{0}<1$ and for $\delta \in\left(0, \delta_{0}\right)$ the series (5.57) is convergent for any $k$.

Having established the existence and a number of properties of $\mathcal{X}^{t}$, in Section 6 we prove in an abstract setup a number of results on vectorfields. After a preliminary section on the spaces $\Sigma_{\ell}$, in Section 8 we check that it is possible to apply the theory in Section [ to appropriate generalizations of $\mathcal{X}^{t}$.

\section{Some Results on ABSTRACT ODE's}

We collect a number of results needed for the Darboux Theorem and the method of normal forms. We will consider a system

$$
\dot{x}=f(t, x), \quad x(0)=\underline{x} .
$$

We assume the following setup:

(1) We consider five separable Hilbert spaces $\mathbb{E}_{i}$ with $i=0,4$ s.t. $\mathbb{E}_{i} \subset \mathbb{E}_{i+1}$ for $i<4, \mathbb{E}_{i}$ is dense in $\mathbb{E}_{i+1}$ and the immersion $j^{(i)}$ of $\mathbb{E}_{i}$ in $\mathbb{E}_{i+1}$ is compact. We denote by $(,)_{i}$, resp. \|\|$_{i}$, the inner product, resp. the norm, in $\mathbb{E}_{i}$.

(2) We assume the existence of $\left\{j_{\epsilon}: \epsilon>0\right\} \subset B\left(\mathbb{E}_{i+1}, \mathbb{E}_{i}\right)$ for $i=0, \ldots, 3$ s.t.: $\left\|j_{\epsilon} \circ j^{(i)}\right\|_{B\left(\mathbb{E}_{i}, \mathbb{E}_{i}\right)} \leq C$ for fixed $C$ for all $\epsilon>0 ; \lim _{\epsilon \searrow 0} j^{(i)} \circ j_{\epsilon}=\mathbb{1}_{\mathbb{E}_{i+1}}$ in $B\left(\mathbb{E}_{i+1}, \mathbb{E}_{i+1}\right) ; \lim _{\epsilon \backslash 0} j_{\epsilon} \circ j^{(i)}=\mathbb{1}_{\mathbb{E}_{i}}$ in $B\left(\mathbb{E}_{i}, \mathbb{E}_{i}\right)$.

(3) Let $\mathfrak{B}_{i}$ be the neighborhood of $0 \in \mathbb{E}_{i}$ defined by $\|x\|_{4}<c_{0}$, for some fixed $c_{0}>0$. Then $f \in C^{n}\left((-3,3) \times \mathfrak{B}_{i}, \mathbb{E}_{i+1}\right)$ for an $n \geq 1$.

(4) The following inequalities hold for a positive constant $C(\lambda)$ which are increasing functions of $\lambda$ :

$$
\begin{gathered}
\|f(t, x)\|_{i+1} \leq C\left(\|x\|_{4}\right)\|x\|_{i}, \\
\left|\left(j_{\epsilon} f(t, x), x\right)_{i}\right| \leq C\left(\|x\|_{4}\right)\|x\|_{i}^{2} \forall \epsilon \in(0,1) \text { and } i, \\
\left|\left(\partial_{x}^{k} j_{\epsilon} f(t, x)(u, v), v\right)_{i+1}\right| \leq C\left(\|x\|_{i}\right)\|u\|_{\mathbb{E}_{i}^{k-1}}\|v\|_{i+1}^{2} \\
\forall 1 \leq k \leq n, \epsilon>0, i=0, \ldots, 3 \text { and } v \in \mathbb{E}_{i} .
\end{gathered}
$$

The main results of this section are Propositions 6.1 and 6.2

Proposition 6.1. $\exists$ a neighborhood $\mathcal{U}$ of 0 in $\mathfrak{B}_{0} \subseteq \mathbb{E}_{0}$ s.t. $\forall \underline{x} \in \mathcal{U}$ system (6.1) has exactly one solution $x(t) \in \bigcap_{i=1}^{2} C^{i-1}\left([-2,2], \mathbb{E}_{i}\right)$. $\mathcal{U}$ can be chosen to be defined by $\|\underline{x}\|_{4}<\varepsilon_{0}$. For $\varepsilon_{0}>0$ small enough we have for a fixed $C$

$$
\begin{aligned}
\|x\|_{L^{\infty}\left([-2,2], \mathbb{E}_{0}\right)} & \leq C\|\underline{x}\|_{0}, \quad\|x\|_{W^{1, \infty}\left([-2,2], \mathbb{E}_{1}\right)} \leq C\|\underline{x}\|_{0}, \\
\|x\|_{L^{\infty}\left([-2,2], \mathbb{E}_{4}\right)} & \leq C\|\underline{x}\|_{4} .
\end{aligned}
$$

Furthermore, denoting by $\phi^{t}$ the flow associated to (6.1), we have $\phi^{t}(\underline{x}) \in C([-2,2]$, $\left.C^{n}\left(\mathcal{U}, \mathbb{E}_{2}\right)\right)$.

We will also use a second version of the above result. 
Proposition 6.2. Assume that hypotheses (1)-(4) hold, but only with four spaces $\mathbb{E}_{i}$ with $i=0,3$ and with \|\|$_{3}$ replacing the \|\|$_{4}$ norm. Then there exists an $\varepsilon_{0}>0$ such that if $\mathcal{U}$ is the subset of $\mathbb{E}_{0}$ defined by $\|\underline{x}\|_{3}<\varepsilon_{0}$, system (6.1) has exactly one solution $x(t) \in \bigcap_{i=1}^{2} C^{i-1}\left([-2,2], \mathbb{E}_{i}\right)$. The following inequalities hold for a fixed $C$ :

$$
\begin{aligned}
\|x\|_{L^{\infty}\left([-2,2], \mathbb{E}_{0}\right)} & \leq C\|\underline{x}\|_{0}, \quad\|x\|_{W^{1, \infty}\left([-2,2], \mathbb{E}_{1}\right)} \leq C\|\underline{x}\|_{0}, \\
\|x\|_{L^{\infty}\left([-2,2], \mathbb{E}_{3}\right)} & \leq C\|\underline{x}\|_{3} .
\end{aligned}
$$

Furthermore, if we denote by $\phi^{t}$ the flow associated to (6.1), then $\partial_{t}^{i} \phi^{t}(\underline{x}) \in C([-2,2]$, $\left.C^{n}\left(\mathcal{U}, \mathbb{E}_{2+i}\right)\right)$ for $i=0,1$.

Proof. The proof is the same as that of Proposition 6.1 with one minor modification. That is, in Lemma 6.3 below, set $X=\mathbb{E}_{3}$ instead of $X=\mathbb{E}_{4}$. The corresponding inequalities and their proofs are exactly the same.

Proof of Proposition 6.1. The proof is tailored on standard arguments (see [Ta] after p. 360), but in the absence of an obvious reference we review it.

We consider systems

$$
\dot{x}_{\epsilon}=j_{\epsilon} f\left(t, x_{\epsilon}\right), \quad x_{\epsilon}(0)=\underline{x} .
$$

We have the following lemma.

Lemma 6.3. There is $\varepsilon_{0}>0$ s.t., $\forall \underline{x} \in \mathbb{E}_{0}$ with $\|\underline{x}\|_{4}<\varepsilon_{0}$, system (6.7) has exactly one solution $x_{\epsilon}(t) \in C^{1}\left([-2,2], \mathbb{E}_{0}\right)$. In particular there is a fixed $C$ s.t. for all $\epsilon \in(0,1)$,

$$
\begin{aligned}
& \left\|x_{\epsilon}\right\|_{L^{\infty}\left([-2,2], \mathbb{E}_{0}\right) \cap W^{1, \infty}\left([-2,2], \mathbb{E}_{1}\right)} \leq C\|\underline{x}\|_{0}, \\
& \left\|x_{\epsilon}\right\|_{L^{\infty}\left([-2,2], \mathbb{E}_{4}\right)} \leq C\|\underline{x}\|_{4} .
\end{aligned}
$$

Proof. By hypothesis we have $\left\|j_{\epsilon} \partial_{x}^{k} f(t, x)\right\|_{0} \leq C(\epsilon, x)$ for all $k=0, \ldots, n$ and $x \in \mathfrak{B}_{0}$. This implies that for $\underline{x} \in \mathfrak{B}_{0}$ the conclusions of Lemma 6.3 hold in some interval $\left(-a_{\epsilon}(\underline{x}), a_{\epsilon}(\underline{x})\right)$. We want to show that $a_{\epsilon}(\underline{x})>2$ if $\|\underline{x}\|_{X}<\varepsilon_{0}$ for $\varepsilon_{0}>0$ small enough, where $X=\mathbb{E}_{4}$. We consider by (6.3)

$$
\left|\frac{d}{d t}\left\|x_{\epsilon}\right\|_{0}^{2}\right|=2\left|\left(j_{\epsilon} f\left(t, x_{\epsilon}\right), x_{\epsilon}\right)_{0}\right| \leq 2 C\left(\left\|x_{\epsilon}\right\|_{X}\right)\left\|x_{\epsilon}\right\|_{0}^{2} .
$$

By Gronwall we get $\left\|x_{\epsilon}\right\|_{0} \leq e^{|t| C(1)}\|\underline{x}\|_{0}$ as long as $\left\|x_{\epsilon}\right\|_{X} \leq 1$ in $[-|t|,|t|]$. We claim that the latter holds for $|t| \leq \frac{1-\log \varepsilon_{0}}{C(1)}$. By (6.3) we have

$$
\frac{d}{d t}\left\|x_{\epsilon}\right\|_{X}^{2}=2\left(j_{\epsilon} f\left(t, x_{\epsilon}\right), x_{\epsilon}\right)_{X} \leq 2 C\left(\left\|x_{\epsilon}\right\|_{X}\right)\left\|x_{\epsilon}\right\|_{X}^{2} .
$$

For $\varepsilon_{0}$ small enough, using Gronwall we obtain (6.8) and $|t| \geq 2$. By equation (6.7) and by (6.2) we have $\left\|\dot{x}_{\epsilon}\right\|_{1} \leq C^{\prime}\|\underline{x}\|_{0}$ for some fixed $C^{\prime}$. So Lemma 6.3 is proved.

We now exploit Lemma 6.3 to prove that the first part of Proposition 6.1 estimates (6.5). The argument is routine. Given a sequence $\epsilon_{\nu} \searrow 0$, then $\left\{x_{\epsilon_{\nu}}\right\}$ admits a subsequence convergent in $C\left([-2,2], \mathbb{E}_{1}\right)$ by Ascoli Arzéla. We can assume it is the whole sequence. We denote the limit by $x(t)$. Since we have necessarily $x_{\epsilon_{\nu}} \rightarrow x$ in $C\left([-2,2], \mathbb{E}_{j}\right)$ for $j>1$, (6.8) yields the third inequality in (6.5). Notice that $x(t)$ is the weak limit of $x_{\epsilon_{\nu}}(t)$ in $\mathbb{E}_{0}$ for any $|t| \leq 2$. By Fatou this yields the first inequality in (6.5). We have $f\left(t, x_{\epsilon_{\nu}}\right) \rightarrow f(t, x)$ in $C\left([-2,2], \mathbb{E}_{2}\right)$. We claim we have 
$j_{\epsilon_{\nu}} f\left(t, x_{\epsilon_{\nu}}\right) \rightarrow f(t, x)$ in $C\left([-2,2], \mathbb{E}_{2}\right)$. Indeed, by $\lim _{\epsilon \searrow} j_{\epsilon}=j$ in $B\left(\mathbb{E}_{1}, \mathbb{E}_{2}\right)$ and by $\left\|f\left(t, x_{\epsilon_{\nu}}\right)\right\|_{\mathbb{E}_{1}} \leq C\|\underline{x}\|_{\mathbb{E}_{0}}$, it follows that in $\mathbb{E}_{2}$ and uniformly in $t \in[-2,2]$, we have

$$
j_{\epsilon_{\nu}} f\left(t, x_{\epsilon_{\nu}}\right)=\left(j_{\epsilon_{\nu}}-j\right) f\left(t, x_{\epsilon_{\nu}}\right)+j^{(1)} f\left(t, x_{\epsilon_{\nu}}\right) \rightarrow f(t, x) .
$$

This implies $\dot{x} \in C\left([-2,2], \mathbb{E}_{2}\right)$, which is the limit of $\dot{x}_{\epsilon_{\nu}}$ and satisfies equation (6.1). By Fatou as before this yields the second inequality in (6.5). Suppose $y \in$ $\bigcap_{i=1}^{2} C^{i-1}\left([-2,2], \mathbb{E}_{i}\right)$ is a solution of (6.1). Then by (6.4) for $k=1$ we have for $\delta x=y-x$

$$
\frac{d\|\delta x\|_{2}^{2}}{d t}=2 \int_{0}^{1}\left(\partial_{x} f(t,(1-\tau) x+\tau y) \delta x, \delta x\right)_{2} \leq C\left(\|x\|_{1},\|y\|_{1}\right)\|\delta x\|_{2}^{2} .
$$

Notice indeed that, by $\lim _{\epsilon}{ }_{0} j_{\epsilon}=j^{(i)}$ in $B\left(\mathbb{E}_{i}, \mathbb{E}_{i+1}\right)$, (6.4) implies

$$
\left|\left(\partial_{x}^{k} f(t, x)(u, v), v\right)_{i+1}\right| \leq C\left(\|x\|_{i}\right)\|u\|_{\mathbb{E}_{i}^{k-1}}\|v\|_{i+1}^{2} .
$$

By Gronwall, (6.9) implies $x \equiv y$. This concludes the proof of the first part of Proposition 6.1 and of (6.5).

We now turn to the proof of the last sentence of Proposition 6.1 $\mathcal{U}$ will be the neighborhood of 0 in $\mathbb{E}_{0}$ defined by $\|\underline{x}\|_{4}<\varepsilon_{0}$. We have proved that $\phi^{t}(\underline{x})=$ $\lim _{\epsilon \searrow 0} \phi_{\epsilon}^{t}(\underline{x})$ in $C\left([-2,2], \mathbb{E}_{1}\right)$, with $\phi_{\epsilon}^{t}$ the flow associated to (6.7). We have $\phi_{\epsilon}^{t}(\underline{x}) \in$ $C^{n}\left([-2,2] \times \mathcal{U}, \mathbb{E}_{0}\right)$.

Lemma 6.4. $\phi^{t} \in C\left(\mathcal{U}, \mathbb{E}_{1}\right)$ for all $t \in[-2,2]$.

Proof. Given $\underline{x}, \underline{y} \in \mathcal{U}$, set $\delta \phi_{\epsilon}=\phi_{\epsilon}^{t}(\underline{x})-\phi_{\epsilon}^{t}(\underline{y})$. By (6.4) and the first part of Proposition 6.1.

$$
\frac{d}{d t}\left\|\delta \phi_{\epsilon}\right\|_{1}^{2}=2\left(j_{\epsilon}\left(f\left(t, \phi_{\epsilon}^{t}(\underline{x})\right)-f\left(t, \phi_{\epsilon}^{t}(\underline{y})\right)\right), \delta \phi_{\epsilon}\right)_{1} \leq C\left\|\delta \phi_{\epsilon}\right\|_{1}^{2} .
$$

This implies $\left\|\phi_{\epsilon}^{t}(\underline{x})-\phi_{\epsilon}^{t}(\underline{y})\right\|_{1} \leq C^{\prime}\|\underline{x}-\underline{y}\|_{1}$ for a fixed $C^{\prime}$. For $\epsilon \searrow 0$ this yields for a fixed $C^{\prime \prime}$

$$
\left\|\phi^{t}(\underline{x})-\phi^{t}(\underline{y})\right\|_{1} \leq C^{\prime}\|\underline{x}-\underline{y}\|_{1} \leq C^{\prime \prime}\|\underline{x}-\underline{y}\|_{0} .
$$

Lemma 6.5. We have $\left\|\partial_{\underline{y}}^{l} \phi_{\epsilon}^{t}(\underline{y})\right\|_{B^{l}\left(\mathbb{E}_{0}, \mathbb{E}_{1}\right)}+\left\|\partial_{t} \partial_{\underline{y}}^{l} \phi_{\epsilon}^{t}(\underline{y})\right\|_{B^{l}\left(\mathbb{E}_{0}, \mathbb{E}_{2}\right)} \leq C$ for a fixed $C$ and for all $1 \leq l \leq n, \underline{y} \in \overline{\mathcal{U}}$ and $\epsilon \in(0,1)$.

Proof. We have

$$
\partial_{t} \partial_{\underline{y}} \phi_{\epsilon}^{t}(\underline{y})=j_{\epsilon} \partial_{\phi} f\left(t, \phi_{\epsilon}^{t}(\underline{y})\right) \partial_{\underline{y}} \phi_{\epsilon}^{t}(\underline{y}), \quad \partial_{\underline{y}} \phi_{\epsilon}^{0}(\underline{y})=\mathbb{1} .
$$

For $l>1$ we have $\partial_{\underline{y}}^{l} \phi_{\epsilon}^{0}(\underline{y})=0$ and, succinctly,

$$
\begin{aligned}
& \partial_{t} \partial_{\underline{y}}^{l} \phi_{\epsilon}^{t}(\underline{y})=j_{\epsilon} \partial_{\phi} f\left(t, \phi_{\epsilon}^{t}(\underline{y})\right) \partial_{\underline{y}}^{l} \phi_{\epsilon}^{t}(\underline{y}) \\
& +\operatorname{Sym} \sum_{k=2}^{l} \sum_{|\alpha|=l} j_{\epsilon} \partial_{\phi}^{k} f\left(t, \phi_{\epsilon}^{t}(\underline{y})\right) \frac{l !}{\alpha !} \partial_{\underline{y}}^{\alpha_{1}} \phi_{\epsilon}^{t}(\underline{y}) \ldots \partial_{\underline{y}}^{\alpha_{k}} \phi_{\epsilon}^{t}(\underline{y}),
\end{aligned}
$$

with $|\alpha|=\sum_{j} \alpha_{j}$ and $\alpha !=\prod_{j} \alpha_{j}$ ! and Sym an appropriate symmetrization; see [N], p. 7. Fix $v \in \mathbb{E}_{0}$. By (6.4) and for $\partial_{\underline{y}} \phi_{\epsilon}^{t}=\partial_{\underline{y}} \phi_{\epsilon}^{t}(\underline{y})$,

$$
\frac{d}{d t}\left\|\partial_{\underline{y}} \phi_{\epsilon}^{t} v\right\|_{1}^{2}=2\left(j_{\epsilon} \partial_{\phi} f\left(t, \phi_{\epsilon}^{t}\right) \partial_{\underline{y}} \phi_{\epsilon}^{t} v, \partial_{\underline{y}} \phi_{\epsilon}^{t} v\right)_{1} \leq C\left\|\partial_{\underline{y}} \phi_{\epsilon}^{t} v\right\|_{1}^{2} .
$$


Since $C$ is independent of $v$, we obtain $\left\|\partial_{y} \phi_{\epsilon}^{t}(y)\right\|_{B\left(\mathbb{E}_{0}, \mathbb{E}_{1}\right)} \leq C_{1}$ for some fixed $C_{1}$ by Gronwall. We assume $\left\|\partial_{y}^{k} \phi_{\epsilon}^{t}(\underline{y})\right\|_{B^{k}\left(\mathbb{E}_{0}, \mathbb{E}_{1}\right)} \leq C$ for $k<l$ by induction. Let $K(t, \epsilon, \underline{y})$ be the second line of $(\underline{6.13})$. Then $\|K(t, \epsilon, \underline{y})\|_{B^{l}\left(\mathbb{E}_{0}, \mathbb{E}_{1}\right)} \leq C$ by induction. Fix $v \in \mathbb{E}_{0}^{l}$. Then, proceeding as for $l=1$ we get

$$
\frac{d}{d t}\left\|\partial_{\underline{y}}^{l} \phi_{\epsilon}^{t}(\underline{y}) v\right\|_{1}^{2} \leq C\left\|\partial_{\underline{y}}^{l} \phi_{\epsilon}^{t}(\underline{y}) v\right\|_{1}+C\|K(t, \epsilon, \underline{y}) v\|_{1} .
$$

By Gronwall we get $\left\|\partial_{\underline{y}}^{l} \phi_{\epsilon}^{t}(\underline{y}) v\right\|_{1} \leq C_{l}\|v\|_{\mathbb{E}_{0}^{l}}$, and so $\left\|\partial_{\underline{y}}^{l} \phi_{\epsilon}^{t}(\underline{y}) v\right\|_{B^{l}\left(\mathbb{E}_{0}, \mathbb{E}_{1}\right)} \leq C_{l}$ since the constants $C$ in (6.15) and $C_{l}$ do not depend on $v$. By equations (6.12) -6.13) we obtain $\left\|\partial_{t} \partial_{\underline{y}}^{l} \phi_{\epsilon}^{t}(\underline{y}) v\right\|_{B^{l}\left(\mathbb{E}_{0}, \mathbb{E}_{2}\right)} \leq C_{l}^{\prime}$.

The natural embedding $B^{l}\left(\mathbb{E}_{i}, \mathbb{E}_{i+1}\right) \hookrightarrow B^{l}\left(\mathbb{E}_{i}, \mathbb{E}_{i+2}\right)$ is compact. This implies that for any fixed $y$ and any sequence $\epsilon_{\nu} \searrow 0$ there is a subsequence $\partial_{y}^{l} \phi_{\epsilon_{\nu}}^{t}(\underline{y})$ convergent in $C\left([-2,2], B^{l}\left(\mathbb{E}_{0}, \mathbb{E}_{2}\right)\right)$ to a $g^{(l)}(t, y)$. $\partial_{t} g^{(1)}(t, \underline{y})$ exists by (6.12), with $\partial_{t} \partial_{\underline{y}} \phi_{\epsilon_{\nu}}^{t}(\underline{y})$ convergent to it in $C\left([-2,2], B\left(\mathbb{E}_{0}, \mathbb{E}_{3}\right)\right)$ and with

$$
\partial_{t} g^{(1)}(t, \underline{y})=\partial_{\phi} f\left(t, \phi^{t}(\underline{y})\right) g^{(1)}(t, \underline{y}), \quad g^{(1)}(0, \underline{y})=\mathbb{1} .
$$

Suppose that $g_{1}^{(1)}(t, y)$ and $g_{2}^{(1)}(t, y)$ are two such solutions of (6.16). Fix $v \in \mathbb{E}_{0}$ and set $\delta g(t, \underline{y})=g_{1}^{(1)}(t, \underline{y})-g_{2}^{(1)}(t, \underline{y})$. Then

$$
\frac{d}{d t}\|\delta g(t, \underline{y}) v\|_{3}^{2}=2\left(\partial_{\phi} f\left(t, \phi^{t}(\underline{y})\right) \delta g(t, \underline{y}) v, \delta g(t, \underline{y}) v\right)_{3} \leq C\|\delta g(t, \underline{y}) v\|_{3}^{2},
$$

by (6.4) and the first part of Proposition 6.1. This implies $\delta g(t, \underline{y}) v=0$ and so $g_{1}^{(1)}(t, \underline{y})=g_{2}^{(1)}(t, \underline{y})$. For $l>1$, by induction and a similar argument, we get a function $g^{(l)}(t, \underline{y}) \in C\left([-2,2], B^{l}\left(\mathbb{E}_{0}, \mathbb{E}_{2}\right)\right)$ satisfying $g^{(l)}(0, \underline{y})=0$ and such that $\partial_{t} g^{(l)}(t, \underline{y}) \in C\left([-2,2], B^{l}\left(\mathbb{E}_{0}, \mathbb{E}_{3}\right)\right)$ satisfies

$$
\begin{aligned}
& \partial_{t} g^{(l)}(t, \underline{y})=\partial_{\phi} f\left(t, \phi^{t}(\underline{y})\right) g^{(l)}(t, \underline{y}) \\
& +\operatorname{Sym} \sum_{k=2}^{l} \sum_{|\alpha|=l} j_{\epsilon} \partial_{\phi}^{k} f\left(t, \phi_{\epsilon}^{t}(\underline{y})\right) \frac{l !}{\alpha !} g^{\left(\alpha_{1}\right)}(t, \underline{y}) \ldots g^{\left(\alpha_{k}\right)}(t, \underline{y}),
\end{aligned}
$$

where in the second line we have only $g^{(\ell)}(t, \underline{y})$ with $\ell<l$, which can be assumed uniquely defined by induction. By repeating the previous argument we also get uniqueness for $k=l$.

Lemma 6.6. For any $t$ the map $\phi^{t}: \mathcal{U} \rightarrow \mathbb{E}_{2}$ is Fréchet differentiable with $\partial_{\underline{y}} \phi^{t}(\underline{y})=g^{(1)}(t, \underline{y})$. We have $\partial_{t}^{i} \partial_{\underline{y}} \phi^{t} \in C\left([-2,2] \times \mathcal{U}, B\left(\mathbb{E}_{0}, \mathbb{E}_{i+2}\right)\right)$ for $i=0,1$.

Proof. For fixed $\underline{x}, \underline{y} \in \mathcal{U}$ set $\delta \underline{x}=\underline{y}-\underline{x}$ and $\delta \phi=\phi^{t}(\underline{y})-\phi^{t}(\underline{x})$. Then

$$
\partial_{t}\left[\delta \phi-g^{(1)}(t, \underline{x}) \delta \underline{x}\right]=f\left(t, \phi^{t}(\underline{y})\right)-f\left(t, \phi^{t}(\underline{x})\right)-\partial_{\phi} f\left(t, \phi^{t}(\underline{x})\right) g^{(1)}(t, \underline{x}) \delta \underline{x} .
$$

Then, for $g^{(1)}=g^{(1)}(t, \underline{x})$, we have

$$
\begin{aligned}
& \frac{d}{d t}\left\|\delta \phi-g^{(1)} \delta \underline{x}\right\|_{2}^{2}=2\left(\partial_{\phi} f\left(t, \phi^{t}(\underline{x})\right)\left(\delta \phi-g^{(1)} \delta \underline{x}\right), \delta \phi-g^{(1)} \delta \underline{x}\right)_{2} \\
& +\int_{0}^{1}\left(\partial_{\phi}^{2} f\left(t,(1-\tau) \phi^{t}(\underline{x})+\tau \phi^{t}(\underline{y})\right)(\delta \phi)^{2}, \delta \phi-g^{(1)} \delta \underline{x}\right)_{2} d \tau .
\end{aligned}
$$


The last line is less than $C\|\delta \phi\|_{1}^{2}\left\|\delta \phi-g^{(1)} \delta \underline{x}\right\|_{2}$. Hence by (6.11)

$$
\begin{aligned}
& \frac{d}{d t}\left\|\delta \phi-g^{(1)} \delta \underline{x}\right\|_{2} \leq C\left\|\delta \phi-g^{(1)} \delta \underline{x}\right\|_{2}+C\|\delta \phi\|_{1}^{2} \\
& \leq C\left\|\delta \phi-g^{(1)} \delta \underline{x}\right\|_{2}+C_{1}\|\delta \underline{x}\|_{1}^{2} .
\end{aligned}
$$

This yields $\left\|\delta \phi-g^{(1)} \delta \underline{x}\right\|_{2}=o\left(\|\delta \underline{x}\|_{0}\right)$. Hence $\phi^{t}: \mathcal{U} \rightarrow \mathbb{E}_{2}$ is Fréchet differentiable with $\partial_{y} \phi^{t}(\underline{y})=g^{(1)}(t, \underline{y})$. Set $\delta g^{(1)}=g^{(1)}(t, \underline{y})-g^{(1)}(t, \underline{x})$ and set $\delta \underline{x}=\underline{y}-\underline{x}$ as above. Then

$$
\partial_{t} \delta g^{(1)}=\partial_{\phi} f\left(t, \phi^{t}(\underline{y})\right) g^{(1)}(t, \underline{y})-\partial_{\phi} f\left(t, \phi^{t}(\underline{x})\right) g^{(1)}(t, \underline{x}) .
$$

Then for fixed $v \in \mathbb{E}_{0}$

$$
\begin{aligned}
& \frac{d}{d t}\left\|\delta g^{(1)} v\right\|_{2}^{2}=2\left(\partial_{\phi} f\left(t, \phi^{t}(\underline{x})\right) \delta g^{(1)} v, \delta g^{(1)} v\right)_{2} \\
& +2\left(\left(\partial_{\phi} f\left(t, \phi^{t}(\underline{y})\right)-\partial_{\phi} f\left(t, \phi^{t}(x)\right) g^{(1)}(t, \underline{y}) v, \delta g^{(1)} v\right)_{2} .\right.
\end{aligned}
$$

By (6.10), for a fixed $C$ we have

$$
\frac{d}{d t}\left\|\delta g^{(1)} v\right\|_{2} \leq C\left\|\delta g^{(1)} v\right\|_{2}+C\|\delta \underline{x}\|_{0}\|v\|_{0} .
$$

Hence $\left\|g^{(1)}(t, \underline{y})-g^{(1)}(t, \underline{x})\right\|_{B\left(\mathbb{E}_{0}, \mathbb{E}_{2}\right)} \leq C^{\prime}\|\underline{y}-\underline{x}\|_{0}$ for a fixed $C^{\prime}$. This and $g^{(1)}(t, \underline{x}) \in C\left([-2,2], B\left(\mathbb{E}_{0}, \mathbb{E}_{2}\right)\right)$ for all $\underline{x}$ imply $g^{(1)} \in C\left([-2,2] \times \mathcal{U}, B\left(\mathbb{E}_{0}, \mathbb{E}_{2}\right)\right)$. We obtain $\partial_{t} g^{(1)} \in C\left([-2,2] \times \mathcal{U}, B\left(\mathbb{E}_{0}, \mathbb{E}_{3}\right)\right)$ by $(\underline{6.16})$.

Lemma 6.7. $\partial_{y}^{l-1} \phi^{t}: \mathcal{U} \rightarrow \mathbb{E}_{2}$ is Fréchet differentiable for any $t$ and $1 \leq l \leq n$ with $\partial_{\underline{y}}^{l} \phi^{t}(\underline{y})=g^{(l)}(t, \underline{y})$. We have $\partial_{t}^{i} \partial_{\underline{y}}^{l} \phi^{t} \in C\left([-2,2] \times \mathcal{U}, B^{l}\left(\mathbb{E}_{0}, \mathbb{E}_{i+2}\right)\right)$ for $i=0,1$.

Proof. Case $l=1$ is proved in Lemma 6.6. Consider $l>1$. By induction we can assume: $\phi^{t}(\underline{x})$ admits Fréchet derivatives of order $k<l ; \partial_{\underline{x}}^{k} \phi^{t}(\underline{x})=g^{(k)}(t, \underline{x})$; $\partial_{t}^{i} g^{(k)} \in C\left([-2,2] \times \mathcal{U}, B^{k}\left(\mathbb{E}_{0}, \mathbb{E}_{i+2}\right)\right)$ for $i=0,1$. Now fix $\underline{x}, \underline{y} \in \mathcal{U}$ and $v \in \mathbb{E}_{0}^{l-1}$. Set $\delta \underline{x}=\underline{y}-\underline{x}, g^{(l)}=g^{(l)}(t, \underline{x})$ and $\delta g^{(l-1)}=g^{(l-1)}(t, \underline{y})-g^{(l-1)}(t, \underline{x})$. Then

$$
\begin{aligned}
& \partial_{t}\left[\delta g^{(l-1)} v-g^{(l)}(v, \delta \underline{x})\right]=\partial_{\phi} f\left(t, \phi^{t}(\underline{x})\right)\left[\delta g^{(l-1)} v-g^{(l)}(v, \delta \underline{x})\right] \\
& \left.+\left(\partial_{\phi} f\left(t, \phi^{t}(\underline{y})\right)-\partial_{\phi} f\left(t, \phi^{t}(\underline{x})\right)\right) \partial_{y}^{l-1} \phi^{t}(\underline{y}) \cdot v+F(z)\right]_{z=\underline{x}}^{z=} \cdot v \\
& -\operatorname{Sym} \sum_{k=2}^{l} \sum_{|\alpha|=l} \partial_{\phi}^{k} f\left(t, \phi^{t}(\underline{x})\right) \frac{l !}{\alpha !} \partial_{\underline{x}}^{\alpha_{1}} \phi^{t}(\underline{x}) \ldots \partial_{\underline{x}}^{\alpha_{k}} \phi^{t}(\underline{x}) \cdot(v, \delta \underline{x})
\end{aligned}
$$

where $|\alpha|=\sum_{j=1}^{k} \alpha_{j}, \alpha !=\prod_{j=1}^{k} \alpha_{j}$ ! and where

$$
F(z):=\operatorname{Sym} \sum_{k=2}^{l-1} \sum_{|\alpha|=l-1} \partial_{\phi}^{k} f\left(t, \phi^{t}(z)\right) \frac{(l-1) !}{\alpha !} \partial_{z}^{\alpha_{1}} \phi^{t}(z) \ldots \partial_{z}^{\alpha_{k}} \phi^{t}(z) .
$$

Notice that $\left|\alpha_{j}\right| \leq l-1$ (resp. $\left|\alpha_{j}\right| \leq l-2$ ) for all $j$ in (6.18) (resp. (6.19)). The last two lines in (6.18) can be written as

$$
\begin{aligned}
& F(z)]_{z=\underline{x}}^{z=\underline{y}} \cdot v+o(\delta \underline{x})+\partial_{\phi}^{2} f\left(t, \phi^{t}(\underline{x})\right)\left(\partial_{\underline{x}} \phi^{t}(\underline{x}) \delta \underline{x}\right) \partial_{\underline{x}}^{l-1} \phi^{t}(\underline{x}) \cdot v \\
& -\operatorname{Sym} \sum_{k=2}^{l} \sum_{|\alpha|=l} \partial_{\phi}^{k} f\left(t, \phi^{t}(\underline{x})\right) \frac{l !}{\alpha !} \partial_{\underline{x}}^{\alpha_{1}} \phi^{t}(\underline{x}) \ldots \partial_{\underline{x}}^{\alpha_{k}} \phi^{t}(\underline{x}) \cdot(v, \delta \underline{x}),
\end{aligned}
$$


where $\|o(\delta \underline{x})\|_{2}=\|v\|_{\mathbb{E}_{0}^{l-1}} o\left(\|\delta \underline{x}\|_{0}\right)$. The last term in the first line cancels with a corresponding one in the second. What remains in the last lines of (6.20) is $-\left(F^{\prime}(\underline{x}) \delta \underline{x}\right) \cdot v$, where we know by induction that $F(z)$ is Fréchet differentiable. Hence all of (6.20) is $o(\delta \underline{x})$. Then by (6.4) and Gronwall we get

$$
\left\|\delta g^{(l-1)}-g^{(l)}(t, \underline{x}) \delta \underline{x}\right\|_{B^{l-1}\left(\mathbb{E}_{0}, \mathbb{E}_{2}\right)}=o\left(\|\delta \underline{x}\|_{0}\right) .
$$

So $g^{(l-1)}(t, \cdot): \mathcal{U} \rightarrow \mathbb{E}_{2}$ is Fréchet differentiable and $\partial_{x} g^{(l-1)}(t, \underline{x})=g^{(l)}(t, \underline{x})$. By the usual argument one shows that $\left\|g^{(l)}(t, y)-g^{(l)}(t, \underline{x})\right\|_{B^{l}\left(\mathbb{E}_{0}, \mathbb{E}_{2}\right)} \leq C^{\prime}\|y-\underline{x}\|_{0}$ for a fixed $C^{\prime}$. This and $g^{(l)}(t, \underline{x}) \in C\left([-2,2], B^{l}\left(\mathbb{E}_{0}, \mathbb{E}_{2}\right)\right)$ for fixed $\underline{x}$ imply that $g^{(l)} \in C\left([-2,2] \times \mathcal{U}, B^{l}\left(\mathbb{E}_{0}, \mathbb{E}_{2}\right)\right)$. We obtain $\partial_{t} g^{(l)} \in C\left([-2,2] \times \mathcal{U}, B^{l}\left(\mathbb{E}_{0}, \mathbb{E}_{3}\right)\right)$ by (6.17).

The last statement of Proposition 6.1 is proved.

\section{Some Facts on the SPACES $\Sigma_{l}$}

We need some preliminary information on the space $\Sigma_{\ell}$ defined by the norm (1.8). Consider the space $\Sigma_{\ell}^{\prime}$ with norm

$$
\|U\|_{\Sigma_{\ell}^{\prime}}^{2}:=\|U\|_{H^{n}}^{2}+\sum_{|\alpha| \leq \ell}\left\|x^{\alpha} U\right\|_{L^{2}}^{2}<\infty
$$

Lemma 7.1. We have $\Sigma_{\ell}=\Sigma_{\ell}^{\prime} . \Sigma_{\ell}$ is preserved by the flow of (2.6).

Proof. The second statement follows from the first by the fact that $\Sigma_{\ell}^{\prime}$ is preserved by the flow of (2.6); see [MS].

The fact that $\Sigma_{\ell}^{\prime} \subseteq \Sigma_{n}$ follows from Proposition 2 of [MS]. We prove $\Sigma_{\ell}^{\prime} \supseteq \Sigma_{\ell}$ by induction. Set $T_{a}:=\mathrm{i} \partial_{a}+\mathrm{i} x_{a}$. We have $T_{a}^{*} T_{a}=-\partial_{a}^{2}+x_{a}^{2}-1$. Then

$$
\|U\|_{\Sigma_{1}}^{2} \approx\|U\|_{L^{2}}^{2}+\left\langle\left(-\Delta+|x|^{2}\right) U \mid \bar{U}\right\rangle \approx\|U\|_{\Sigma_{1}^{\prime}}^{2}
$$

by the fact that $\langle(-\Delta+1) U \mid \bar{U}\rangle$ defines $H^{1}$. So $\Sigma_{1}=\Sigma_{1}^{\prime}$. Suppose by induction that $\Sigma_{\ell-1}=\Sigma_{\ell-1}^{\prime}$. Let $|\alpha|=\ell-1$. Then

$$
\begin{aligned}
& \left\|\partial_{x}^{\alpha} U\right\|_{H^{1}}^{2} \lesssim\left\|\partial_{x}^{\alpha} U\right\|_{L^{2}}^{2}+\left\langle\left(-\Delta+|x|^{2}\right) \partial_{x}^{\alpha} U \mid \partial_{x}^{\alpha} \bar{U}\right\rangle \approx\left\|\partial_{x}^{\alpha} U\right\|_{L^{2}}^{2} \\
& +\sum_{a=1}^{3}\left\|\left(\mathrm{i} \partial_{a}+\mathrm{i} x_{a}\right) \partial_{x}^{\alpha} U\right\|_{L^{2}}^{2} \leq\left\|\partial_{x}^{\alpha} U\right\|_{L^{2}}^{2} \\
& +\sum_{a=1}^{3}\left\|\partial_{x}^{\alpha}\left(\mathrm{i} \partial_{a}+\mathrm{i} x_{a}\right) U\right\|_{L^{2}}^{2}+\sum_{a=1}^{3}\left\|\left[\mathrm{i} \partial_{a}+\mathrm{i} x_{a}, \partial_{x}^{\alpha}\right] U\right\|_{L^{2}}^{2} \lesssim\|U\|_{\Sigma_{\ell}}^{2},
\end{aligned}
$$

by induction and by $\sum_{a=1}^{3}\left\|\left[\mathrm{i} \partial_{a}+\mathrm{i} x_{a}, \partial_{x}^{\alpha}\right] U\right\|_{L^{2}} \lesssim\|u\|_{H^{\ell-1}}$. For the same $\alpha$, using the Fourier transform we see that (7.3) also implies

$$
\sum_{a=1}^{3}\left\|x_{a} x^{\alpha} U\right\|_{L^{2}}^{2} \lesssim\|U\|_{\Sigma_{\ell}}^{2} .
$$

Then (7.3)-(7.4) imply $\|U\|_{\Sigma_{\ell}^{\prime}} \lesssim\|U\|_{\Sigma_{\ell}}$ and so $\Sigma_{\ell} \subseteq \Sigma_{\ell}^{\prime}$. Since we already know that $\Sigma_{\ell} \supseteq \Sigma_{\ell}^{\prime}$, they are equal.

Definition 7.2. For any positive integer $\ell$ we denote by $\Sigma_{-\ell}$ the space formed by the $V$ such that the map $U \rightarrow\langle U \mid \bar{V}\rangle$ is in $B\left(\Sigma_{\ell}, \mathbb{C}\right)$. We also set $\Sigma_{0}:=L^{2}$. 
Definition 7.2 yields a natural Banach structure on $\Sigma_{-\ell}$. Notice that we have found two distinct but equivalent norms in $\Sigma_{\ell}$. A third one comes from claim (4) in Lemma 7.3. In the subsequent proofs we will pick in the proofs from time to time the norms which are most convenient, with the statements independent from the particular choice. There will also be a corresponding implicit choice of inner products.

Lemma 7.3. Set $h=-\Delta+|x|^{2}$. The following facts hold:

(1) The map $(h+1)^{\ell}: \Sigma_{\ell} \rightarrow \Sigma_{-\ell}$ is an isomorphism between $\Sigma_{\ell}$ and $\Sigma_{-\ell}$ for any $\ell \in \mathbb{N}$.

(2) $\|U\|_{\Sigma_{\ell}} \approx\left\|(h+1)^{\frac{\ell}{2}} U\right\|_{L^{2}}$ for any $\ell \in \mathbb{Z}$.

(3) The map $(h+1)^{\frac{j}{2}}: \Sigma_{\ell} \rightarrow \Sigma_{\ell-j}$ is an isomorphism for any $(\ell, j) \in \mathbb{Z}^{2}$.

(4) The map $(h+1)^{\frac{j}{2}}: \Sigma_{\ell} \rightarrow \Sigma_{\ell-j}$ is an isomorphism for any $(\ell, j) \in \mathbb{R}^{2}$ if we define $\Sigma_{\ell}$ for $\ell \in \mathbb{R}$ setting $\|U\|_{\Sigma_{\ell}}:=\left\|(h+1)^{\frac{\ell}{2}} U\right\|_{L^{2}}$.

Proof. The proof of (1) is an easy consequence of $T_{a}^{*} T_{a}=-\partial_{a}^{2}+x_{a}^{2}-1$ and $\left[T_{a}, T_{b}^{*}\right]=$ $2 \delta_{a b}$ and is skipped. Proofs of the other claims are elementary.

We will consider the following mollifier:

$$
J_{\varepsilon}=\left(1-\varepsilon \Delta+\varepsilon|x|^{2}\right)^{-1} \text { for } \varepsilon>0 .
$$

Lemma 7.4. Let $s>0$. The following facts hold:

(1) Denote by $j: \Sigma_{\ell} \rightarrow \Sigma_{\ell-s}$ the natural embedding. Then $\lim _{\epsilon} \searrow_{0} J_{\epsilon}^{\frac{s}{2}}=j$ in $B\left(\Sigma_{\ell}, \Sigma_{\ell-s}\right)$.

(2) We have $J_{\epsilon}^{\frac{s}{2}}: \Sigma_{\ell} \rightarrow \Sigma_{\ell+s}$ with $\left\|J_{\epsilon}^{\frac{s}{2}}\right\|_{B\left(\Sigma_{\ell}, \Sigma_{\ell+s}\right)} \leq C \epsilon^{-\frac{s}{2}}$.

Proof. Set $h=-\Delta+|x|^{2}$. For any $U \in \Sigma_{\ell}$ there is a fixed $C$ s.t.

$$
\begin{aligned}
& \left\|\left(1-J_{\epsilon}^{\frac{s}{2}}\right) U\right\|_{\Sigma_{\ell-s}}=\left\|(h+1)^{\frac{\ell-s}{2}}\left(1-J_{\epsilon}^{\frac{s}{2}}\right) U\right\|_{L^{2}} \\
& =\left\|(h+1)^{\frac{\ell-s}{2}}\left(1-\left(1-\frac{\epsilon h}{\epsilon h+1}\right)^{\frac{s}{2}}\right) U\right\|_{L^{2}} \leq C \epsilon\left\|(h+1)^{\frac{\ell}{2}} U\right\|_{L^{2}} .
\end{aligned}
$$

So $J_{\epsilon}^{\frac{s}{2}}-j=O(\epsilon)$ in $B\left(\Sigma_{\ell}, \Sigma_{\ell-s}\right)$. The second claim follows by the Spectral Theorem and $(1+r)^{\frac{\ell+s}{2}}(1+\epsilon r)^{-\frac{s}{2}} \leq \epsilon^{-\frac{s}{2}}(1+r)^{\frac{\ell}{2}}$ for any $r \geq 0$.

Lemma 7.5. For any $\ell \in \mathbb{Z}$ we have $x_{a}, \partial_{a} \in B\left(\Sigma_{\ell}, \Sigma_{\ell-1}\right)$.

Proof. For $\ell>0, f \in \Sigma_{\ell}$ and $|\alpha| \leq \ell-1$ we have

$$
\left\|(\mathrm{i} \partial+\mathrm{i} x)^{\alpha} \partial_{a} f\right\|_{L^{2}} \leq\left\|\partial_{a}(\mathrm{i} \partial+\mathrm{i} x)^{\alpha} f\right\|_{L^{2}}+\left\|\left[(\mathrm{i} \partial+\mathrm{i} x)^{\alpha}, \partial_{a}\right] f\right\|_{L^{2}} \leq C\|f\|_{\Sigma_{\ell}},
$$

where we are using (7.3) and the fact that $\left[(\mathrm{i} \partial+\mathrm{i} x)^{\alpha}, \partial_{a}\right]$ is a linear combination of $(\mathrm{i} \partial+\mathrm{i} x)^{\beta}$ with $|\beta|=|\alpha|-1$. The case with $x_{a}$ is seen to be equivalent, through the Fourier transform. The case with $\ell \leq 0$ follows by duality.

Lemma 7.6. There is a fixed $C>0$ s.t. $\forall \varepsilon \in(0,1)$ for $T=x_{a}, \partial_{a}$ and $\forall \ell \in \mathbb{Z}$ we have $\varepsilon\left(\left\|T J_{\varepsilon}\right\|_{B\left(\Sigma_{\ell}, \Sigma_{\ell}\right)}+\left\|J_{\varepsilon} T\right\|_{B\left(\Sigma_{\ell}, \Sigma_{\ell}\right)}\right)<C$.

Proof. By Lemma 7.4 and Lemma [7.5, for $T=x_{a}, \partial_{a}$ we have $\epsilon\left\|J_{\epsilon} T f\right\|_{\Sigma_{\ell}} \leq$ $C\|T f\|_{\Sigma_{\ell-1}} \leq C^{\prime}\|f\|_{\Sigma_{\ell}}$. Similarly $\epsilon\left\|T J_{\epsilon} f\right\|_{\Sigma_{\ell}} \leq C \epsilon\left\|J_{\epsilon} f\right\|_{\Sigma_{\ell+1}} \leq C^{\prime}\|f\|_{\Sigma_{\ell}}$.

Lemma 7.7. For any $\ell \in \mathbb{Z}$ and $n \in \mathbb{N}$ there is a fixed $C>0$ s.t. $\forall \varepsilon \in(0,1)$ we have $\left\|\left[T, J_{\varepsilon}^{n}\right]\right\|_{B\left(\Sigma_{2 \ell}, \Sigma_{2 \ell}\right)}<C$ with $T=x_{a}, \partial_{a}$. 
Proof. We have $\left[\partial_{a}, J_{\varepsilon}^{n}\right]=-(\epsilon h+1)^{-n}\left[\partial_{a},(\epsilon h+1)^{-n}\right](\epsilon h+1)^{-n}$. It is elementary that this is a sum of terms $-2 \epsilon(\epsilon h+1)^{j-n} x_{a}(\epsilon h+1)^{k-n}$ with $j+k=n-1$. Then for $\ell \geq 0$,

$$
\begin{aligned}
& \left\|\epsilon(\epsilon h+1)^{j-n} x_{a}(\epsilon h+1)^{k-n} f\right\|_{\Sigma_{\ell}} \leq C\left\|\epsilon x_{a}(\epsilon h+1)^{k-n} f\right\|_{\Sigma_{\ell}} \\
& \leq C_{1}\left\|\epsilon(\epsilon h+1)^{k-n} f\right\|_{\Sigma_{\ell+1}} \leq C_{2}\left\|(\epsilon h+1)^{k-n+1} f\right\|_{\Sigma_{\ell}} \leq C_{3}\|f\|_{\Sigma_{\ell} .}
\end{aligned}
$$

The other estimates can be proved similarly. The case $\ell<0$ follows by duality.

Lemma 7.8. For any $n \in \mathbb{N}$ there is a fixed $C>0$ s.t. $\forall \varepsilon \in(0,1)$ we have $\left\|\left[T, J_{\varepsilon}^{n}\right]\right\|_{B\left(H^{1}, H^{1}\right)}<C$ with $T=x_{a}, \partial_{a}$.

Proof. We know that $\left\|\left[T, J_{\varepsilon}^{n}\right]\right\|_{B\left(L^{2}, L^{2}\right)}<C$ from Lemma 7.7 for $\ell=0$. Proceeding as above we need to show that terms like the following ones are in $B\left(H^{1}, L^{2}\right)$ :

$$
\begin{aligned}
& \epsilon \partial_{b}(\epsilon h+1)^{j-n} x_{a}(\epsilon h+1)^{k-n}=\epsilon\left[\partial_{b},(\epsilon h+1)^{j-n}\right] x_{a}(\epsilon h+1)^{k-n} \\
& +\delta_{a b} \epsilon(\epsilon h+1)^{-n-1}+\epsilon(\epsilon h+1)^{j-n} x_{a}\left[\partial_{b},(\epsilon h+1)^{k-n}\right] \\
& +\epsilon(\epsilon h+1)^{j-n} x_{a}(\epsilon h+1)^{k-n} \partial_{b} .
\end{aligned}
$$

All the terms on the rhs except for the last one are in $B\left(L^{2}, L^{2}\right)$ with norm bounded uniformly in $\epsilon$. For the last one the same holds in $B\left(H^{1}, L^{2}\right)$.

Lemma 7.9. Let $\ell \in \mathbb{Z}$ and let $n \in \mathbb{N}$ such that $n+\ell \geq 1$. Then there are fixed constants $C_{\ell}$ and $C$ such that for $T=\partial_{a}, x_{a}$,

$$
\left|\left(f, J_{\epsilon}^{2 n} T f\right)_{\Sigma_{2 \ell}}\right| \leq C\|f\|_{\Sigma_{2 \ell}}^{2}, \quad\left|\left(f, J_{\epsilon}^{2 n} T f\right)_{H^{1}}\right| \leq\|f\|_{H^{1}}^{2} .
$$

Proof. Set $h=-\Delta+|x|^{2}$ and $X=\Sigma_{2 \ell}, H^{1}$. Then

$$
\begin{aligned}
& \left((\epsilon h+1)^{-2 n} \partial_{a} f, f\right)_{X}=\left(\left[(\epsilon h+1)^{-n}, \partial_{a}\right] f,(\epsilon h+1)^{-n} f\right)_{X} \\
& +\left(\partial_{a}(\epsilon h+1)^{-n} f,(\epsilon h+1)^{-n} f\right)_{X} .
\end{aligned}
$$

We have

$$
\left|\left(\left[(\epsilon h+1)^{-n}, \partial_{a}\right] f,(\epsilon h+1)^{-n} f\right)_{X}\right| \leq\|f\|_{X}\left\|\left[(\epsilon h+1)^{-n}, \partial_{a}\right] f\right\|_{X} \leq C\|f\|_{X}^{2}
$$

by Lemmas 7.7 and 7.8 , We have for $\ell \in \mathbb{Z}$

$$
\begin{aligned}
& \left(\partial_{a}(\epsilon h+1)^{-n} f,(\epsilon h+1)^{-n} f\right)_{\Sigma_{2 \ell}} \\
& =\left((h+1)^{\ell} \partial_{a}(\epsilon h+1)^{-n} f,(h+1)^{\ell}(\epsilon h+1)^{-n} f\right)_{L^{2}} \\
& =\left(\left[(h+1)^{\ell}, \partial_{a}\right](\epsilon h+1)^{-n} f,(h+1)^{\ell}(\epsilon h+1)^{-n} f\right)_{L^{2}},
\end{aligned}
$$

where we exploited $\left(\partial_{a} g, g\right)_{L^{2}}=\left(\sigma_{3} x_{a} g, g\right)_{L^{2}}=0$ for $\bar{g}=\sigma_{1} g$. The rhs is in absolute value less than $\|f\|_{\Sigma_{\ell}}^{2}$. The proof for the case $X=H^{1}$ is simpler.

\section{Quasilinear SYSTEMS}

We will apply the theory developed in Section [ in two distinct forms to quasilinear systems

$$
\begin{aligned}
& \dot{f}=\mathcal{L} f+\mathcal{D}, \quad \dot{z}=Z, \\
& \mathcal{L}:=\mathcal{A}_{a} \partial_{a}+\left(\mathcal{B}_{a} x_{a}+\mathcal{C}\right) \sigma_{3},
\end{aligned}
$$

with $\mathcal{L}, \mathcal{D}$ and $Z$ satisfying hypotheses which we will state below. 
8.1. First type of system. We consider $4 n_{i}=4 n_{0}-i 4 n \geq n+1 \gg 1$ with $i=0,1,2,3$. We denote $\mathbb{E}_{i}=\mathbb{C}^{m} \times P_{c}\left(\omega_{0}\right) \Sigma_{4 n_{i}}$ with $i=0,1,2,3$. Also set $\mathbb{E}_{4}=\mathbb{C}^{m} \times P_{c}\left(\omega_{0}\right) H^{1}$ and $j_{\epsilon}=J_{\epsilon}^{2 n}$. We assume:

(A1) $\mathcal{A}_{a}$ are real valued functions. $\mathcal{B}_{a}$ and $\mathcal{C}$ are imaginary valued. $Z_{\underline{j}}$ has values in $\mathbb{C}$ with $Z_{\bar{j}}=\bar{Z}_{j}$.

(A2) $\mathcal{D}$ has values in $\Sigma_{4 n_{0}}$. For $G=\mathcal{A}_{a}, \mathcal{B}_{a}, \mathcal{C}, \mathcal{D}, Z_{\bar{j}}$ we have $G=G(t, z, f, \varrho(f))$, where $G(t, z, f, \varrho)$ is $C^{n}$ in $t, z$, in $f \in \Sigma_{-4 n_{0}}$ and in $\rho$.

(A3) We have

$$
\begin{aligned}
& \left|\mathcal{A}_{a}\right|+|Z|+|\mathcal{C}|+\|\mathcal{D}\|_{\Sigma_{4 n_{0}}} \\
& \leq C\left(|z|+\|f\|_{\Sigma_{-4 n_{0}}}+|\varrho(f)|\right)\left(|z|+\|f\|_{\Sigma_{-4 n_{0}}}\right) .
\end{aligned}
$$

(A4) We have either $\mathcal{B}_{a}=-\frac{\mathrm{i}}{2} v_{a}-\frac{\mathrm{i}}{2} t d v_{a}\left(\mathcal{X}^{t}\right)$ for $a=1,2,3$ or $\mathcal{B}_{a} \equiv 0$.

The coefficients of Lemma 5.8 satisfy (A1)-(A4) for any choice of $n$ and $n_{0}$, by our freedom of choice of space $H^{K, S}$ and by the fact that $H^{K, S} \subset \Sigma_{4 n_{0}}$ for $K>4 n_{0}$ and $S>4 n_{0}$. We have:

Proposition 8.1. The following facts hold:

(1) $\exists$ a neighborhood $\mathcal{U}$ of $0 \in \mathbb{E}_{0}$ defined by $|z|<\varepsilon_{0}$ and $\|f\|_{H^{1}}<\varepsilon_{0}$, s.t. $\forall(\underline{z}, f) \in \mathcal{U}$ system (6.1) has exactly one solution $(z(t), f(t)) \in$ $\cap_{i=1}^{2}\left(C^{i-1}\left([-2,2], \mathbb{E}_{i}\right) \cap W^{i-1, \infty}\left([-2,2], \mathbb{E}_{i-1}\right)\right)$.

(2) Call $\phi^{t}$ the flow of (8.1). Then $\partial_{t}^{i} \phi^{t} \in C^{i}\left([-2,2], C^{n}\left(\mathcal{U}, \mathbb{E}_{2+i}\right)\right)$ for $i=0,1$.

(3) For $\left(z^{t}, f^{t}\right)=\phi^{t}(z, f)$ we have

$$
\begin{aligned}
& \left\|z^{t}\right\|_{L^{\infty}(-2,2)}+\left\|f^{t}\right\|_{L^{\infty}\left([-2,2], H^{1}\right)} \leq C\left(|z|+\|f\|_{H^{1}}\right), \\
& \left\|f^{t}\right\|_{L^{\infty}\left([-2,2], \Sigma_{4 n_{0}}\right)} \leq C\left(|z|+\|f\|_{\Sigma_{4 n_{0}}}\right) .
\end{aligned}
$$

Proof. We will need to check that we are in the framework and the hypotheses of Section [6. Proposition 8.1 is a consequence of Proposition 6.1] if we can prove hypotheses (1)-(4) in Section 6. Specifically we need to prove the inequalities in (4) of Section 6. By hypotheses (A3)-(A4) and by Lemma 7.5 we immediately see that the analogue of (6.2) is satisfied. (6.3) is a consequence of the following lemma.

Lemma 8.2. For a fixed constant $C$ and for $C_{|z|+|\varrho(f)|}$ an increasing positive function in $|z|+|\varrho(f)|$, we have:

$$
\begin{aligned}
& |Z \cdot \bar{z}| \leq C|z|\left(|z|+\|f\|_{\Sigma_{-4 n_{0}}}+|\varrho(f)|\right)\left(|z|+\|f\|_{\Sigma_{-4 n_{0}}}\right), \\
& \left|(f, \mathcal{D})_{\Sigma_{4 n_{i}}}\right| \leq C\|f\|_{\Sigma_{4 n_{i}}}\left(|z|+\|f\|_{\Sigma_{-4 n_{0}}}+|\varrho(f)|\right)\left(|z|+\|f\|_{\Sigma_{-4 n_{0}}}\right), \\
& \left|(f, \mathcal{D})_{H^{1}}\right| \leq C\|f\|_{L^{2}}\left(|z|+\|f\|_{\Sigma_{-4 n_{0}}}+|\varrho(f)|\right)\left(|z|+\|f\|_{\Sigma_{-4 n_{0}}}\right), \\
& \left|\left(f, J_{\epsilon}^{2 n} \mathcal{L} f\right)_{\Sigma_{4 n_{i}}}\right| \leq C_{|z|+|\varrho(f)|}\|f\|_{\Sigma_{4 n_{i}}}^{2}, \\
& \left|\left(f, J_{\epsilon}^{2 n} \mathcal{L} f\right)_{H^{1}}\right| \leq C_{|z|+|\varrho(f)|} \mid\|f\|_{H^{1}}^{2} .
\end{aligned}
$$

Proof. The first three inequalities follow immediately from (A2)-(A3). The last two inequalities are an immediate consequence of the following two inequalities for $\widehat{T}=\mathbb{1}, \partial_{a}, x_{a}$ for any $a=1,2,3$ : there is a fixed $C$ s.t.

$$
\left|\left(f, J_{\epsilon}^{2 n} \widehat{T} f\right)_{\Sigma_{4 n_{i}}}\right| \leq C\|f\|_{\Sigma_{4 n_{i}}}^{2}, \quad\left|\left(f, J_{\epsilon}^{2 n} \widehat{T} f\right)_{H^{1}}\right| \leq C\|f\|_{H^{1}}^{2} .
$$

(8.4) follows from Lemma 7.9 
Finally, to finish with the proof of Proposition 6.1] we need to prove that (6.4) is true. But like (6.3) this too is an easy consequence of (A2)-(A4) and of Lemma 7.9. Hence Proposition 8.1 is proved.

8.2. Second type of system. Before setting up the system we notice that for solutions of (8.1) satisfying (A1)-(A4) we have

$$
\begin{aligned}
& \frac{d}{d t} Q(f)=\left\langle\sigma_{1} f \mid \dot{f}\right\rangle=\left\langle\sigma_{1} f \mid \mathcal{D}\right\rangle, \\
& \frac{d}{d t} \Pi_{a}(f)=\mathrm{i}\left\langle\sigma_{1} \sigma_{3} \partial_{a} f \mid \dot{f}\right\rangle=\mathrm{i} \mathcal{B}_{b}\left\langle\sigma_{1} \sigma_{3} \partial_{a} f \mid \sigma_{3} x_{b} f\right\rangle+\mathrm{i}\left\langle\sigma_{1} \sigma_{3} \partial_{a} f \mid \mathcal{D}\right\rangle \\
& =2 \mathrm{i} Q(f) \mathcal{B}_{a}-\mathrm{i}\left\langle\sigma_{1} \sigma_{3} f \mid \partial_{a} \mathcal{D}\right\rangle .
\end{aligned}
$$

We set $\varrho_{0}(f):=Q(f)$ and $\varrho_{a}(f):=\Pi_{a}(f)$. We consider the system

$$
\begin{aligned}
& \dot{\varrho}_{0}=\left\langle\sigma_{1} f \mid \mathcal{D}\right\rangle, \quad \dot{\varrho}_{a}=2 \mathrm{i} \varrho_{0} \mathcal{B}_{a}-\mathrm{i}\left\langle\sigma_{1} \sigma_{3} f \mid \partial_{a} \mathcal{D}\right\rangle, \\
& \dot{f}=\mathcal{L} f+\mathcal{D}, \quad \dot{z}=Z .
\end{aligned}
$$

We denote $\mathbb{E}_{i}=\mathbb{R}^{4} \times \mathbb{C}^{m} \times P_{c}\left(\omega_{0}\right) \Sigma_{-4 n_{3-i}}$ with $i=0,1,2,3$. We assume:

(B1) the same as (A1);

(B2) $\mathcal{D}$ has values in $\Sigma_{4 n_{3}}$. For $G=\mathcal{A}_{a}, \mathcal{B}_{a}, \mathcal{C}, \mathcal{D}, Z_{\bar{j}}$ we have $G=G(t, z, f, \varrho)$, where $G(t, z, f, \varrho)$ is $C^{n}$ in $t, z$, in $f \in \Sigma_{-4 n_{0}}$ and in $\rho$;

(B3) we have

$$
\begin{aligned}
& \left|\mathcal{A}_{a}\right| \leq C\left(|z|^{2}+\|f\|_{\Sigma_{-4 n_{0}}}^{2}+|\varrho|\right) \\
& |Z|+|\mathcal{C}|+\|\mathcal{D}\|_{\Sigma_{4 n_{0}}} \leq C\left(|z|+\|f\|_{\Sigma_{-4 n_{0}}}+|\varrho|\right)\left(|z|+\|f\|_{\Sigma_{-4 n_{0}}}\right)
\end{aligned}
$$

(B4) we have either $\left|\mathcal{B}_{a}\right| \leq C|\varrho|$ for $a=1,2,3$ or $\mathcal{B}_{a} \equiv 0$.

Notice that, starting from $E=\mathcal{A}_{a}, \mathcal{B}_{a}, \mathcal{C}, \mathcal{D}, Z_{\bar{j}}$, with $E=E(t, z, f, \varrho(f))$ which satisfy hypotheses (A1)-(A4) in Subsection 8.1 and substituting $\varrho(f)$ with an external parameter $\varrho$, we obtain functions satisfying (B1)-(B4).

We have:

Proposition 8.3. The following facts hold:

(1) $\exists$ a neighborhood $\mathcal{U}$ of $0 \in \mathbb{E}_{0}$ defined by $|\varrho|+|z|<\varepsilon_{0}$ and $\|f\|_{\Sigma_{-4 n_{0}}}<\varepsilon_{0}$, s.t. $\forall(\underline{z}, f) \in \mathcal{U}$ system (6.1) has exactly one solution $(\varrho(t), z(t), f(t)) \in$ $\bigcap_{i=1}^{2}\left(C^{i-1}\left([-2,2], \mathbb{E}_{i}\right) \cap W^{i-1, \infty}\left([-2,2], \mathbb{E}_{i+1}\right)\right)$.

(2) Call $\Phi^{t}$ the flow of (8.6). Then $\partial_{t}^{i} \Phi^{t}(y) \in C\left([-2,2], C^{n}\left(\mathcal{U}, \mathbb{E}_{2+i}\right)\right)$ for $i=$ 0,1 .

(3) Set $\left(z_{t}, f_{t}, \varrho_{t}\right):=\Phi^{t}(z, f, \varrho)$. Then we have for $i=0,3$

$$
\left\|\left(z^{t},\left\|f^{t}\right\|_{\Sigma_{-4 n_{i}}}, \varrho^{t}\right)\right\|_{L^{\infty}(-2,2)} \leq C\left(|z|+\|f\|_{\Sigma_{-4 n_{i}}}+|\varrho|\right) .
$$

(4) Call $\phi^{t}$ the flow of (8.1) satisfying (A1)-(A4) and suppose that (8.6) is the corresponding system substituting $\varrho(f)$ with an external parameter $\varrho$. When $f \in H^{1}$ and for $\left(z^{t}, f^{t}\right):=\phi^{t}(z, f)$, we have $\Phi^{t}(\varrho(f), z, f)=\left(\varrho\left(f^{t}\right), z^{t}, f^{t}\right)$.

Proof. As in Subsection 8.1 we can reduce to material in Section 6. Specifically, by the arguments of Subsection 8.1 we can apply Proposition 6.2. This yields the claims (1)-(3). Claim (4) follows from (8.5) and the uniqueness of solutions in (8.6). 
8.3. Structure of the Lie transform. Consider system (8.1) such that (A1)(A4) hold. Consider the corresponding system (8.6) satisfying (B1)-(B4). We denote by $\phi=\phi^{1}$ the Lie transform.

Lemma 8.4. Set $\left(z^{\prime}, f^{\prime}\right)=\phi(z, f)$. Then we have

$$
z^{\prime}=z+\mathcal{Z}, \quad f^{\prime}(x)=e^{\sigma_{3}(\boldsymbol{B} \cdot x+\gamma)} \tau_{\mathbf{A}} f+\mathcal{G}(x)
$$

with $\tau_{\mathbf{A}} f(x)=f(x-\mathbf{A}), \mathbf{A}=-\int_{0}^{1} \mathcal{A}(\tau) d \tau, \mathcal{Z}=\int_{0}^{1} Z(\tau) d \tau, \boldsymbol{B}=\int_{0}^{1} \mathcal{B}(\tau) d \tau$, $\gamma=\int_{0}^{1}\left(\mathcal{C}(s)+\mathcal{A}_{a}(s) \int_{0}^{s} \mathcal{B}_{a}(\tau) d \tau\right) d s$ and $\mathcal{G}$ functions of $(z, f, \varrho(f))$ such that

$$
\mathcal{G}(x)=\int_{0}^{1} e^{-\sigma_{3} \int_{s}^{1}\left(x_{a} \mathcal{B}_{a}(\tau)+\mathcal{C}(\tau)-\mathcal{A}_{a}(\tau) \int_{0}^{\tau} \mathcal{B}_{a}\left(\tau^{\prime}\right) d \tau^{\prime}\right) d \tau} \mathcal{D}\left(s, x+\int_{s}^{1} \mathcal{A}(\tau) d \tau\right) d s .
$$

We have the following estimates for a fixed constant $C>0$ :

$$
\begin{gathered}
\left\|\left(z,\|f\|_{\Sigma_{-4 n_{0}}}\right)\right\|_{L^{\infty}(-2,2)} \leq C\left(|z|+\|f\|_{\Sigma_{-4 n_{0}}}\right), \\
|\mathcal{Z}|+\|\mathcal{G}\|_{\Sigma_{n_{4} 0}} \leq C\left(|z|+\|f\|_{\Sigma_{-4 n_{0}}}+|\varrho|\right)\left(|z|+\|f\|_{\Sigma_{-4 n_{0}}}\right), \\
|\boldsymbol{A}|+|\boldsymbol{B}|+|\gamma| \leq C\left(|z|^{2}+\|f\|_{\Sigma_{-4 n_{0}}}^{2}+|\varrho|\right) .
\end{gathered}
$$

We have $\mathcal{Z}=\mathcal{Z}(z, f, \varrho(f)), \boldsymbol{A}=\boldsymbol{A}(z, f, \varrho(f)), \boldsymbol{B}=\boldsymbol{B}(z, f, \varrho(f))$ and $\gamma=\gamma(z, f, \varrho(f))$, with $C^{n}$ dependence in $z \in \mathbb{C}^{m}, f \in \Sigma_{-4 n_{3}}$ and $\varrho \in \mathbb{R}^{4} . \mathcal{G}=\mathcal{G}(z, f, \varrho(f))$ has $C^{n}$ dependence in $z \in \mathbb{C}^{m}, f \in \Sigma_{-4 n_{3}}$ and $\varrho \in \mathbb{R}^{4}$ with values in $\Sigma_{4 n_{0}-n}$. Here $n_{0}=n_{3}+2 n$ and $4 n_{3} \geq n+1$.

Proof. The formulas follow by the use of the integrating factor. The last claim follows from Proposition 8.3. We now consider the estimates (8.10)-(8.12), which improve (8.8). Notice that

$$
z^{t}=z+\mathcal{Z}_{t}, \quad f^{t}(x)=e^{\sigma_{3}\left(\mathbf{B}_{t} \cdot x+\gamma_{t}\right)} \tau_{\mathbf{A}_{t}} f+\mathcal{G}_{t}(x),
$$

defined similarly to (8.9) but with integrals in $[0, t]$ (resp. $[s, t]$ ) rather than in $[0,1]$ (resp. [s, 1]). We have by (8.7)-(8.8)

$$
\left|\mathcal{Z}_{t}\right| \leq \int_{0}^{t}|Z(\tau)| d \tau \leq C\left(|z|+\|f\|_{\Sigma_{-4 n_{0}}}+|\varrho(f)|\right) \int_{0}^{t}\left(\left|z^{\tau}\right|+\left\|f^{\tau}\right\|_{\Sigma_{-4 n_{0}}}\right) d \tau .
$$

Similarly

$$
\int_{0}^{t}|\mathcal{C}(\tau)| d \tau \leq C\left(|z|+\|f\|_{\Sigma_{-4 n_{0}}}+|\varrho(f)|\right) \int_{0}^{t}\left(\left|z^{\tau}\right|+\left\|f^{\tau}\right\|_{\Sigma_{-4 n_{0}}}\right) d \tau .
$$

For $\Upsilon=\mathcal{A}, \mathcal{B}$ we have $\int_{s}^{t}|\Upsilon(\tau)| d \tau \leq C\left(|z|+\|f\|_{\Sigma_{-4 n_{0}}}+|\varrho(f)|\right)^{2}$. As a consequence we get $\left\|e^{\sigma_{3}\left(\mathbf{B}_{t} \cdot x+\gamma_{t}\right)} \tau_{\mathbf{A}_{t}} f\right\|_{\Sigma_{-4 n_{0}}} \leq C\|f\|_{\Sigma_{-4 n_{0}}}$ and

$$
\begin{aligned}
& \left\|\mathcal{G}_{t}\right\|_{\Sigma_{4 n_{0}}} \leq C \int_{0}^{t}\left\|\mathcal{D}\left(s, x+\int_{s}^{t} \mathcal{A}(\tau) d \tau\right)\right\|_{\Sigma_{4 n_{0}}} d s \leq C^{\prime} \int_{0}^{t}\|\mathcal{D}(s, x)\|_{\Sigma_{-4 n_{0}}} d s \\
& \leq C^{\prime \prime}\left(|z|+\|f\|_{\Sigma_{-4 n_{0}}}+|\varrho(f)|\right) \int_{0}^{t}\left(\left|z^{s}\right|+\left\|f^{s}\right\|_{\Sigma_{-4 n_{0}}}\right) d s .
\end{aligned}
$$

Then (8.10) follows by Gronwall's inequality and implies (8.11)-(8.12).

Remark 8.5. Notice that the theory in Section 6 entails loss of regularity, in the sense that $\mathbf{A}$ and the other functions are regular in $f \in \Sigma_{-4 n_{3}}$ but not $f \in \Sigma_{-4 n_{0}}$. Since we consider many flows, we have big losses of regularity. Fortunately we consider no more that $2 N+2$ transformations and we have a lot of regularity to begin with. 
Lemma 8.6. Consider the system $\dot{f}=\left(\mathcal{X}^{t}\right)_{f}$ and $\dot{z}_{j}=\left(\mathcal{X}^{t}\right)_{j}$. Then the conclusions of Lemma 8.4 continue to hold and we also have

$$
f^{\prime}(x)=e^{\sigma_{3}\left(-\frac{i}{2} v^{\prime} \cdot(x-\mathbf{A})+\widetilde{\gamma}\right)} f(x-\mathbf{A})+\mathcal{G}(x)
$$

with $v^{\prime}$ the velocity associated to the $t=1$ vector.

Proof. The starting point is formula (8.9). By Lemma 5.8 we have

$$
\mathbf{B}=\int_{0}^{1} \mathcal{B}_{a}(t) d t=-\frac{\mathrm{i}}{2} \int_{0}^{1}\left(v_{a}(t)+t d v_{a}\left(\mathcal{X}^{t}\right)\right) d t=-\frac{\mathrm{i}}{2} v_{a}(1)
$$

Recalling the $\gamma=\int_{0}^{1}\left(\mathcal{C}(s)+\mathcal{A}_{a}(s) \int_{0}^{s} \mathcal{B}_{a}(\tau) d \tau\right) d s$ in Lemma 8.4, we have

$$
\begin{aligned}
& \int_{0}^{1} d s \mathcal{A}_{a}(s) \int_{0}^{s} \mathcal{B}_{a}(\tau) d \tau=-\frac{\mathrm{i}}{2} \int_{0}^{1} \mathcal{A}_{a}(s) v_{a}(s) d s \\
& =\frac{\mathrm{i}}{2} \int_{0}^{1} v_{a}(s) \frac{d}{d s} \mathbf{A}_{a}(s) d s=\frac{\mathrm{i}}{2} \mathbf{A}_{a}(1) v_{a}(1)-\frac{\mathrm{i}}{2} \int_{0}^{1} \mathbf{A}_{a}(s) \frac{d}{d s} v_{a}(s) d s .
\end{aligned}
$$

We get (8.15), setting $\widetilde{\gamma}:=\gamma-\frac{i}{2} \mathbf{A} \cdot v^{\prime}$.

Lemma 8.7. $z^{\prime}$ is $C^{n}$ in $z \in \mathbb{C}^{m}, f \in \Sigma_{-4 n_{3}}$ and $\varrho \in \mathbb{R}^{4}$. $f^{\prime}$ is $C^{n}$ in $z \in \mathbb{C}^{m}$, $f \in \Sigma_{-4 n_{3}}$ and $\varrho \in \mathbb{R}^{4}$ with values in $\Sigma_{-4 n_{3}-n}$, where $4 n_{3} \geq n+1 . \varrho\left(f^{\prime}\right)$ is $C^{n}$ in $z \in \mathbb{C}^{m}, f \in \Sigma_{-4 n_{3}}$ and $\varrho \in \mathbb{R}^{4}$.

Proof. With the notation of Lemmas 8.4 and 8.6, for $\mathcal{Z}, \mathbf{A}, \widetilde{\gamma}=\gamma-\frac{\mathrm{i}}{2} \mathbf{A} \cdot v^{\prime}$ and $\mathcal{G}$ we have the result of Lemma 8.4. So for $z^{\prime}$ the claim follows immediately, while $f^{\prime}$ is a consequence of formula (8.15), the chain rule and Lemma 7.5. We have

$$
\begin{aligned}
& Q\left(f^{\prime}\right)=Q(f)+2\left\langle e^{\sigma_{3}\left(-\frac{i}{2} v^{\prime} \cdot x+\widetilde{\gamma}\right)} f \mid \sigma_{1} \tau_{-\mathbf{A}} \mathcal{G}\right\rangle+Q(\mathcal{G}), \\
& \Pi_{a}\left(f^{\prime}\right)=\Pi_{a}(f)-\frac{v_{a}^{\prime}}{2} Q(f)+\mathrm{i}\left\langle e^{\sigma_{3}\left(-\frac{i}{2} v^{\prime} \cdot x+\widetilde{\gamma}\right)} f \mid \tau_{-\mathbf{A}} \sigma_{1} \sigma_{3} \partial_{a} \mathcal{G}\right\rangle+\Pi_{a}(\mathcal{G}) .
\end{aligned}
$$

By Lemma 8.4 we have that $\sigma_{1} \sigma_{3}^{i} e^{\sigma_{3}\left(\frac{\mathrm{i}}{2} v^{\prime} \cdot x-\widetilde{\gamma}\right)} \tau_{-\mathbf{A}} \partial_{a}^{i} \mathcal{G}$ for $i=0,1$ is $C^{n}$ in $z \in \mathbb{C}^{m}$, $f \in \Sigma_{-4 n_{3}}$ and $\varrho \in \mathbb{R}^{4}$ with values in $\Sigma_{4 n_{0}-n-i}$ with $n_{0}=n_{3}+2 n$. Then for $f \in \Sigma_{-4 n_{3}}$ and by $4 n_{0}-n-i \geq 4 n_{3}$ it follows that the mixed terms are $C^{n}$ in $z \in \mathbb{C}^{m}, f \in \Sigma_{-4 n_{3}}$ and $\varrho \in \mathbb{R}^{4} . Q(\mathcal{G})$ and $\Pi_{a}(\mathcal{G})$ are of the desired type.

\section{Reformulation of (4.4) in the new Coordinates}

Denote by $\mathcal{F}_{t}$ the flow of the system (8.1) associated to the field of Lemma 5.8, We set

$$
H=K \circ \mathcal{F}_{1} .
$$

In the new coordinates (4.4) becomes

$$
\mathrm{i} \dot{z}_{j}=\frac{\partial H}{\partial \bar{z}_{j}}, \quad \mathrm{i} \dot{f}=\sigma_{3} \sigma_{1} \nabla_{f} H
$$

For system (9.2) we prove:

Theorem 9.1. There exist $\varepsilon_{0}>0$ and $C>0$ such that for $|z(0)|+\|f(0)\|_{H^{1}} \leq$ $\epsilon<\varepsilon_{0}$ with $f(0)$ in the Schwartz class, then the corresponding solution of (9.2) is globally defined and there are $f_{ \pm} \in H^{1}$ with $\left\|f_{ \pm}\right\|_{H^{1}} \leq C \epsilon$ and functions $\widehat{\vartheta} \in$ $C^{1}(\mathbb{R}, \mathbb{R})$ and $\widehat{D} \in C^{1}\left(\mathbb{R}, \mathbb{R}^{3}\right)$ such that

$$
\lim _{t \rightarrow \pm \infty}\left\|\tau_{\widehat{D}(t)} e^{\mathrm{i} \widehat{\vartheta}(t) \sigma_{3}} f(t)-e^{\mathrm{i} t \Delta \sigma_{3}} f_{ \pm}\right\|_{H^{1}}=0 .
$$


We have

$$
\lim _{t \rightarrow \infty} z(t)=0
$$

It is possible to write $f(t, x)=A(t, x)+\tilde{f}(t, x)$ with $|A(t, x)| \leq C_{N}(t)\langle x\rangle^{-N}$ for any $N$, with $\lim _{t \rightarrow \infty} C_{N}(t)=0$ and such that for any admissible pair $(p, q)$, i.e. (1.6), we have $\|\widetilde{f}\|_{L_{t}^{p}\left(\mathbb{R}, W_{x}^{1, q}\right)} \leq C \epsilon$.

We now move to the proof of Theorem 9.1. First of all we remark that in the sequel we need that the Hamiltonians and the Lie transforms be sufficiently regular. The amount of regularity needed depends on $N=N_{1}$. We will consider a total of $2(N+1)$ Lie transforms, considering both the implementation of the Darboux Theorem and the Birkhoff normal forms. We need to end up with a final Hamiltonian which is at least $C^{1}$. We can make sure that all the Hamiltonians and Lie transforms are sufficiently regular by picking $H^{K, S}$ with $K \gg 2 N$ and $S \gg 2 N$ in Lemmas 4.5 and 5.8 ,

The first step in the proof of Theorem 9.1 is a preliminary discussion of $H=K$ 。 $\mathcal{F}_{1}$. In Sections 10 12 we implement the method of Birkhoff normal forms, looking for other coordinates. Finally we will settle on the right system of coordinates and in Section 13 we will finally prove the estimates.

Lemma 9.2. Fix a large number $M \in \mathbb{N}$ with $M \gg 2 N$. We have the expansion

$$
H=\psi(\varrho(f))+H_{2}^{(1)}+\mathcal{R}^{(1)}
$$

where $\psi(\varrho)$ is $C^{M}$ in $\varrho$ and where:

(1) We have for $\ell=1$

$$
H_{2}^{(\ell)}=\sum_{\substack{|\mu+\nu|=2 \\ \lambda^{0} \cdot(\mu-\nu)=0}} a_{\mu \nu}^{(\ell)}(\varrho(f)) z^{\mu} \bar{z}^{\nu}+\frac{1}{2}\left\langle\sigma_{3} \mathcal{H}_{\omega_{0}} f \mid \sigma_{1} f\right\rangle .
$$

(2) We have $\mathcal{R}^{(1)}=\widetilde{\mathcal{R}^{(1)}}+\widetilde{\mathcal{R}^{(2)}}$, with

$$
\begin{aligned}
& \widetilde{\mathcal{R}^{(1)}}=\sum_{\substack{|\mu+\nu|=2 \\
\lambda^{0} \cdot(\mu-\nu) \neq 0}} a_{\mu \nu}^{(1)}(\varrho(f)) z^{\mu} \bar{z}^{\nu}+\sum_{|\mu+\nu|=1} z^{\mu} \bar{z}^{\nu}\left\langle\sigma_{1} \sigma_{3} G_{\mu \nu}(\varrho(f)) \mid f\right\rangle, \\
& \widetilde{\mathcal{R}^{(2)}}=\sum_{|\mu+\nu|=3} z^{\mu} \bar{z}^{\nu} a_{\mu \nu}(z, f, \varrho(f))+\sum_{|\mu+\nu|=2} z^{\mu} \bar{z}^{\nu}\left\langle G_{\mu \nu}(z, f, \varrho(f)) \mid \sigma_{3} \sigma_{1} f\right\rangle \\
& +\sum_{d=2}^{4}\left\langle B_{d}(z, f, \varrho(f)) \mid f^{d}\right\rangle+\int_{\mathbb{R}^{3}} B_{5}(x, z, f, f(x), \varrho(f)) f^{5}(x) d x \\
& +\widehat{\mathcal{R}}_{2}^{(1)}(z, f, \varrho(f))+E_{P}(f), \\
& \quad \text { with B B }(0,0,0)=0 \text { and where, both here and in Theorem } 12.1 \text { later, by } \\
& f^{d}(x) \text { we schematically represent d-product of components of } f .
\end{aligned}
$$

(3) At $\varrho(f)=0$ with $\ell=1$

$$
\begin{aligned}
& a_{\mu \nu}^{(\ell)}(0)=0 \text { for }|\mu+\nu|=2 \text { with }(\mu, \nu) \neq\left(\delta_{j}, \delta_{j}\right) \text { for all } j, \\
& a_{\delta_{j} \delta_{j}}^{(\ell)}(0)=\lambda_{j}\left(\omega_{0}\right) \text {, where } \delta_{j}=\left(\delta_{1 j}, \ldots, \delta_{m j}\right), \\
& G_{\mu \nu}(0)=0 \text { for }|\mu+\nu|=1 .
\end{aligned}
$$


These $a_{\mu \nu}^{(\ell)}(\varrho)$ and $G_{\mu \nu}(x, \varrho)$ are $C^{M}$ in all variables with

$$
G_{\mu \nu}(\cdot, \varrho) \in C^{M}\left(\mathbb{R}^{4}, \Sigma_{4 M}\left(\mathbb{R}^{3}, \mathbb{C}^{2}\right)\right) .
$$

(4) For a small neighborhood $\mathrm{U}$ of $(0,0,0)$ in $\mathbb{C}^{m} \times \Sigma_{-4 M} \times \mathbb{R}^{4}$, we have $a_{\mu \nu}(z, \varrho) \in C^{M}(\mathrm{U}, \mathbb{C})$.

(5) $G_{\mu \nu}(\cdot, z, \varrho) \in C^{M}\left(\mathrm{U}, \Sigma_{4 M}\left(\mathbb{R}^{3}, \mathbb{C}^{2}\right)\right)$.

(6) $B_{d}(\cdot, z, f, \varrho) \in C^{M}\left(\mathrm{U}, \Sigma_{4 M}\left(\mathbb{R}^{3}, B\left(\left(\mathbb{C}^{2}\right)^{\otimes d}, \mathbb{C}\right)\right)\right)$, for $2 \leq d \leq 4$.

(7) Let ${ }^{t} \eta=(\zeta, \bar{\zeta})$ for $\zeta \in \mathbb{C}$. Then for $B_{5}(\cdot, z, f, \eta, \varrho)$ we have

$$
\text { for }|l| \leq M,\left\|\nabla_{z, \bar{z}, f, \zeta, \bar{\zeta}, \varrho}^{l} B_{5}(z, f, \eta, \varrho)\right\|_{\Sigma_{4 M}\left(\mathbb{R}^{3}, B\left(\left(\mathbb{C}^{2}\right)^{\otimes 5}, \mathbb{C}\right)\right.} \leq C_{l} .
$$

(8) We have for all indexes and for $\ell=1$

$$
a_{\mu \nu}^{(\ell)}=\bar{a}_{\nu \mu}^{(\ell)}, \quad a_{\mu \nu}=\bar{a}_{\nu \mu}, \quad G_{\mu \nu}=-\sigma_{1} \bar{G}_{\nu \mu} .
$$

$$
\begin{aligned}
& \widehat{\mathcal{R}}_{2}^{(1)} \in C^{M}(\mathrm{U}, \mathbb{R}), \\
& \left|\widehat{\mathcal{R}}_{2}^{(1)}(z, f, \varrho)\right| \leq C\left(|z|+|\varrho|+\|f\|_{\Sigma_{-4 M}}\right)\|f\|_{\Sigma_{-4 M}}^{2} .
\end{aligned}
$$

Proof. We consider the notation of Lemma 8.6. Thanks to Lemma 8.4 and by the freedom of choice of $H^{K, S}$ in Lemmas 4.5] and [5.8, we can assume that $\mathbf{A}$ and $v^{\prime} \in C^{\widetilde{M}}\left(\widetilde{\mathrm{U}}, \mathbb{R}^{3}\right), \mathcal{G} \in C^{\widetilde{M}}\left(\widetilde{\mathrm{U}}, \Sigma_{4 \widetilde{M}}\right)$ and $\widetilde{\gamma} \in C^{\widetilde{M}}(\widetilde{\mathrm{U}}, \mathrm{i} \mathbb{R})$, with $\widetilde{\mathrm{U}}$ a neighborhood of the origin in the space $\varrho(f)=\varrho \in \mathbb{R}^{4}, z \in \mathbb{C}^{m}$ and $f \in \Sigma_{-4 \widetilde{M}}$ and with $\widetilde{M} \gg M$. Having in mind (8.16), we notice that $e^{\sigma_{3}\left(-\frac{i}{2} v^{\prime} \cdot x+\widetilde{\gamma}\right)} \partial_{a}^{i} \tau_{-\mathbf{A}} \mathcal{G} \in C^{\widehat{M}}\left(\widetilde{\mathrm{U}}, \Sigma_{4 \widetilde{M}-4 \widehat{M}-i}\right)$ for $i=0,1$. Set $F:=e^{\sigma_{3}\left(-\frac{i}{2} v^{\prime} \cdot x+\widetilde{\gamma}\right)} \tau_{-\mathbf{A}} \mathcal{G}$. Then we have, for $\varrho=\varrho(f)$ and $G(t)=F(t z, t f, \varrho)$,

$$
\begin{aligned}
& \langle f \mid F\rangle=\left\langle f \mid\left(z_{j} \partial_{j}+\bar{z}_{j} \partial_{\bar{j}}\right) F(0,0, \varrho)\right\rangle \\
& +\left\langle f \mid \partial_{f} F(0,0, \varrho) f\right\rangle+\frac{1}{2} \int_{0}^{1}\left\langle f \mid \frac{d^{2}}{d t^{2}} G(t)\right\rangle d t .
\end{aligned}
$$

The first term on the right is like the $z^{\mu} \bar{z}^{\nu}\left\langle\sigma_{1} \sigma_{3} G_{\mu \nu}(\varrho(f)) \mid f\right\rangle$ in (9.7) with $G_{\mu \nu}(\varrho) \in$ $C^{\widehat{M}-1}\left(\mathbb{R}^{4}, \Sigma_{4 \widetilde{M}-4 \widehat{M}}\right)$. By taking appropriate $\widetilde{M}$ and $\widehat{M}$, the conditions in the statement will hold. The second term on the rhs of (9.11) is like $\widehat{\mathcal{R}}_{2}^{(1)}(z, f, \varrho)$, satisfying (9.10) for appropriate choices of $\widetilde{M}$ and $\widehat{M}$ by (8.11). The last term in (9.11) is of higher order, and again is like $\widehat{\mathcal{R}}_{2}^{(1)}$ or can be absorbed in $\widetilde{\mathcal{R}}^{(2)}$. Similar expansions hold for $Q(\mathcal{G})$ and analogous terms on the rhs of the second equality in (8.16). Expanding like in (8.16) we obtain

$$
\begin{aligned}
& \left(z^{\prime}\right)^{\mu}\left(\bar{z}^{\prime}\right)^{\nu}\left\langle\sigma_{1} \sigma_{3} \underline{G}_{\mu \nu}\left(\varrho\left(f^{\prime}\right)\right) \mid f^{\prime}\right\rangle \\
& =(z+Z)^{\mu}(\bar{z}+\bar{Z})^{\nu}\left\langle\sigma_{1} \sigma_{3} \underline{G}_{\mu \nu}\left(\varrho\left(f^{\prime}\right)\right) \mid \tau_{\mathbf{A}} e^{\sigma_{3}\left(-\frac{i}{2} v^{\prime} \cdot x+\widetilde{\gamma}\right)} f+\mathcal{G}\right\rangle .
\end{aligned}
$$

Analogous formulas hold for $\left\langle\underline{B}_{d}\left(z^{\prime}, \varrho\left(f^{\prime}\right)\right) \mid\left(f^{\prime}\right)^{d}\right\rangle$ for $d=2,3,4$ and $E_{P}\left(f^{\prime}\right)$. With Taylor expansions similar to (9.11) we get in an elementary fashion that (9.12) expands into terms falling in one of the cases in the statement. For $d=5,0 \leq j \leq 5$, and with some exponentials in absorbed $\underline{B}$, schematically, we have terms like

$$
\int_{\mathbb{R}^{3}} \widehat{B}_{5}\left(x+\mathbf{A}, z^{\prime}, e^{\sigma_{3}\left(-\frac{i}{2} v^{\prime} \cdot x+\widetilde{\gamma}\right)} f(x)+\mathcal{G}(x+\mathbf{A}), \varrho\left(f^{\prime}\right)\right) f^{j}(x) \mathcal{G}^{5-j}(x+\mathbf{A}) d x,
$$


which by Taylor expansion and by the fact that $\widetilde{M} \gg M$ can be absorbed in $\widetilde{\mathcal{R}^{(2)}}$. We next look at

$$
\left\langle\sigma_{3} \mathcal{H}_{\omega_{0}} f^{\prime} \mid \sigma_{1} f^{\prime}\right\rangle=\left\langle\left(-\Delta+\omega_{0}\right) f^{\prime} \mid \sigma_{1} f^{\prime}\right\rangle+\left\langle\sigma_{3} V_{\omega_{0}} f^{\prime} \mid \sigma_{1} f^{\prime}\right\rangle .
$$

We have

$$
\begin{aligned}
& \left\langle\left(-\Delta+\omega_{0}\right) f^{\prime} \mid \sigma_{1} f^{\prime}\right\rangle=\left\langle\left(-\Delta+\omega_{0}\right) f \mid \sigma_{1} f\right\rangle+\frac{v^{2}}{2} Q(f)+2 v_{a} \Pi_{a}(f) \\
& +\left\langle\left(-\Delta+\omega_{0}\right) \mathcal{G} \mid \sigma_{1} \mathcal{G}\right\rangle+2\left\langle\left(-\Delta+\omega_{0}\right) \mathcal{G} \mid \sigma_{1} \tau_{\mathbf{A}} e^{\sigma_{3}\left(-\frac{i}{2} v^{\prime} \cdot x+\widetilde{\gamma}\right)} f\right\rangle .
\end{aligned}
$$

In (9.13) the last line can be treated as above and absorbed in the $\mathcal{R}^{(1)}$, while the last two terms of the first line go in part in $\psi(\varrho(f))$ and in part in $\mathcal{R}^{(1)}$ by $v=2 \Pi(R) / Q(U)$. The term $\left\langle\sigma_{3} V_{\omega_{0}} f^{\prime}, \sigma_{1} f^{\prime}\right\rangle$ can be expanded as the sum of $\left\langle\sigma_{3} V_{\omega_{0}} f, \sigma_{1} f\right\rangle$ plus a remainder term treating it as in (9.12).

\section{NORMAL FORMS AND HOMOLOGICAL EQUATION}

We set $\mathcal{H}=\mathcal{H}_{\omega_{0}} P_{c}\left(\mathcal{H}_{\omega_{0}}\right)$. Consider $\mathbb{C}$ valued functions $a_{\mu \nu}^{(\ell)}(\varrho)$ such that $a_{\nu \mu}^{(\ell)} \equiv$ $\overline{a_{\mu \nu}^{(\ell)}}$. We assume that $a_{\mu \nu}^{(\ell)} \in C^{k_{0}}(U, \mathbb{C})$ for $k_{0} \in \mathbb{N}$ a fixed number and $U$ a neighborhood of 0 in $\mathbb{R}^{4}$. Then we set

$$
\begin{gathered}
H_{2}^{(\ell)}(\varrho):=\sum_{\substack{|\mu+\nu|=2 \\
\lambda(0) \cdot(\mu-\nu)=0}} a_{\mu \nu}^{(\ell)}(\varrho) z^{\mu} \bar{z}^{\nu}+\frac{1}{2}\left\langle\sigma_{3} \mathcal{H}_{\omega_{0}} f \mid \sigma_{1} f\right\rangle . \\
\lambda_{j}^{(\ell)}(\varrho):=a_{\delta_{j} \delta_{j}}^{(\ell)}(\varrho), \quad \lambda^{(\ell)}=\left(\lambda_{1}^{(\ell)}, \ldots, \lambda_{m}^{(\ell)}\right) .
\end{gathered}
$$

We assume $\lambda_{j}^{(\ell)}(0)=\lambda_{j}\left(\omega_{0}\right)$ and $a_{\mu \nu}^{(\ell)}(0)=0$ if $(\mu, \nu) \neq\left(\delta_{j}, \delta_{j}\right)$ for all $j$, with $\delta_{j}$ defined in (9.8).

Definition 10.1. A function $Z(z, f, \varrho)$ is in normal form if it is a sum

$$
Z=Z_{0}+Z_{1}
$$

where $Z_{0}$ and $Z_{1}$ are finite sums of the following type:

$$
Z_{1}=\sum_{|\lambda(\varrho) \cdot(\nu-\mu)|>\omega_{0}} z^{\mu} \bar{z}^{\nu}\left\langle\sigma_{1} \sigma_{3} G_{\mu \nu}(\varrho) \mid f\right\rangle
$$

with $G_{\mu \nu}(x, \omega, \varrho) \in C^{k_{0}}\left(U, \Sigma_{k_{1}}\right)$ for fixed $k_{0} \in \mathbb{N}$;

$$
Z_{0}=\sum_{\lambda(0) \cdot(\mu-\nu)=0} a_{\mu \nu}(\varrho) z^{\mu} \bar{z}^{\nu}
$$

and $a_{\mu \nu}(\varrho) \in C^{k_{0}}(U, \mathbb{C})$. We will always assume the symmetries in (4.12).

For $G=G(x)$, by elementary computations we have

$$
\begin{aligned}
& \frac{1}{2}\left\{\left\langle\sigma_{3} \mathcal{H} f \mid \sigma_{1} f\right\rangle,\left\langle\sigma_{1} \sigma_{3} G, f\right\rangle\right\}=-\mathrm{i}\left\langle f \mid \sigma_{1} \sigma_{3} \mathcal{H} G\right\rangle, \\
& \frac{1}{2}\left\{\left\langle\sigma_{3} \mathcal{H} f \mid \sigma_{1} f\right\rangle, Q(f)\right\}=\mathrm{i}\left\langle\mathcal{H} f \mid \sigma_{1} f\right\rangle=-\mathrm{i}\left\langle\beta^{\prime}\left(\phi^{2}\right) \phi^{2} \sigma_{3} f \mid f\right\rangle, \\
& \frac{1}{2}\left\{\left\langle\sigma_{3} \mathcal{H} f \mid \sigma_{1} f\right\rangle, \Pi_{a}(f)\right\}=\left\langle\sigma_{3} \mathcal{H} f \mid \sigma_{1} \partial_{a} f\right\rangle=-\frac{1}{2}\left\langle\sigma_{3}\left(\partial_{a} V_{\omega_{0}}\right) f \mid \sigma_{1} f\right\rangle, \\
& \{f, Q(f)\}=-\mathrm{i} P_{c}\left(\omega_{0}\right) \sigma_{3} f, \quad\left\{f, \Pi_{a}(f)\right\}=P_{c}\left(\omega_{0}\right) \partial_{a} f .
\end{aligned}
$$

We now discuss the homological equations. We start by assuming that $\varrho$ is an external parameter. 
Lemma 10.2. We consider $\chi=\chi(b, B)$ with

$$
\chi(b, B)=\sum_{|\mu+\nu|=M_{0}+1} b_{\mu \nu} z^{\mu} \bar{z}^{\nu}+\sum_{|\mu+\nu|=M_{0}} z^{\mu} \bar{z}^{\nu}\left\langle\sigma_{1} \sigma_{3} B_{\mu \nu} \mid f\right\rangle
$$

for $b_{\mu \nu} \in \mathbb{C}$ and $B_{\mu \nu} \in \Sigma_{2 k_{1}}$ with $k_{1} \in \mathbb{N}$. Here we interpret the polynomial $\chi$ as a function with parameters $b=\left(b_{\mu \nu}\right)$ and $B=\left(B_{\mu \nu}\right)$. Denote by $X_{2 k_{1}}$ the space of the pairs $(b, B)$. Let us also consider given polynomials with $K=K(\varrho)$ and $\widetilde{K}=K(\varrho, b, B)$ where

$$
K(\varrho):=\sum_{|\mu+\nu|=M_{0}+1} k_{\mu \nu}(\varrho) z^{\mu} \bar{z}^{\nu}+\sum_{|\mu+\nu|=M_{0}} z^{\mu} \bar{z}^{\nu}\left\langle\sigma_{1} \sigma_{3} K_{\mu \nu}(\varrho) \mid f\right\rangle,
$$

with $k_{\mu \nu}(\varrho) \in C^{k_{0}}(U, \mathbb{C})$ and $K_{\mu \nu}(\varrho) \in C^{k_{0}}\left(U, \Sigma_{2 k_{1}} \cap P_{c}\left(\omega_{0}\right) L^{2}\right)$ for $U$ a neighborhood of 0 in $\mathbb{R}^{4}$, and let

$$
\begin{aligned}
& \widetilde{K}(\varrho, b, B):=\sum_{|\mu+\nu|=M_{0}+1} \widetilde{k}_{\mu \nu}(\varrho, b, B) z^{\mu} \bar{z}^{\nu} \\
& +\sum_{i=0}^{1} \sum_{a=1}^{3} \sum_{|\mu+\nu|=M_{0}} z^{\mu} \bar{z}^{\nu}\left\langle\sigma_{1} \sigma_{3} \partial_{a}^{i} K_{a \mu \nu}^{i}(\varrho, b, B) \mid f\right\rangle,
\end{aligned}
$$

with $\widetilde{k}_{\mu \nu} \in C^{k_{0}}\left(U \times X_{2 k_{1}}, \mathbb{C}\right)$ and $\widetilde{K}_{a \mu \nu}^{i} \in C^{k_{0}}\left(U \times X_{2 k_{1}}, \Sigma_{2 k_{1}} \cap P_{c}\left(\omega_{0}\right) L^{2}\right)$. Suppose also that the sums (10.8) and (10.9) do not contain terms in normal form and that $\widetilde{K}(0, b, B)=0$. Then there exists a neighborhood $V \subseteq U$ of 0 in $\mathbb{R}^{4}$ and a unique choice of functions $(b(\varrho), B(\varrho)) \in C^{k_{0}}\left(V, X_{2 k_{1}}\right)$ such that for $\chi(\varrho)=\chi(b(\varrho), B(\varrho))$, $\widetilde{K}(\varrho)=\widetilde{K}(\varrho, b(\varrho), B(\varrho))$, we have

$$
\left\{\chi(\varrho), H_{2}(\varrho)\right\}=K(\varrho)+\widetilde{K}(\varrho)+Z(\varrho),
$$

where $Z(\varrho)$ is in normal form and homogeneous of degree $M_{0}+1$ in $(z, \bar{z}, f)$. If the coefficients of $K$ satisfy the symmetries in (4.12), the same is true for $\chi$.

Proof. Summing on repeated indexes, we get

$$
\begin{aligned}
& \left\{H_{2}, \chi\right\}=\mathrm{i} \lambda(\varrho) \cdot(\mu-\nu) z^{\mu} \bar{z}^{\nu} b_{\mu \nu} \\
& +\mathrm{i}\left\langle f \mid \sigma_{1} \sigma_{3}(\lambda(\varrho) \cdot(\mu-\nu)-\mathcal{H}) B_{\mu \nu}\right\rangle+\widehat{K}(\varrho, b, B),
\end{aligned}
$$

where, with an abuse of notation,

$$
\widehat{K}(\varrho, b, B):=\sum_{\mu \nu \mu^{\prime} \nu^{\prime}} a_{\mu \nu}^{(\ell)}(\varrho)\left(b_{\mu^{\prime} \nu^{\prime}}+\left\langle\sigma_{1} \sigma_{3} B_{\mu^{\prime} \nu^{\prime}} \mid f\right\rangle\right)\left\{z^{\mu} \bar{z}^{\nu}, z^{\mu^{\prime}} \bar{z}^{\nu^{\prime}}\right\},
$$

with the sum only on $|\mu+\nu|=2$ with $(\mu, \nu) \neq\left(\delta_{j}, \delta_{j}\right)$ for all $j . \widehat{K}(\varrho, b, B)$ is 0 for $\varrho=0$ and is a homogeneous polynomial of the same type as the ones above. Denote by $\widehat{Z}(\varrho, b, B)$ the sum of its monomials in normal form and set $\mathbf{K}:=\widetilde{K}+\widehat{K}-\widehat{Z}$. We look at

$$
\begin{aligned}
& \mathrm{i} \lambda(\varrho) \cdot(\mu-\nu) z^{\mu} \bar{z}^{\nu} b_{\mu \nu}+z^{\mu} \bar{z}^{\nu} \mathrm{i}\left\langle f \mid \sigma_{1} \sigma_{3}(\lambda(\varrho) \cdot(\mu-\nu)-\mathcal{H}) B_{\mu \nu}\right\rangle \\
& \quad+\mathbf{K}(\varrho, b, B)+K(\varrho)=0,
\end{aligned}
$$

that is, at

$$
\begin{aligned}
& k_{\mu \nu}(\varrho)+\mathbf{k}_{\mu \nu}(\varrho, b, B)+\mathrm{i} b_{\mu \nu} \lambda(\varrho) \cdot(\mu-\nu)=0, \\
& K_{\mu \nu}(\varrho)+\mathbf{K}_{\mu \nu}(\varrho, b, B)-\mathrm{i}(\mathcal{H}-\lambda(\varrho) \cdot(\mu-\nu)) B_{\mu \nu}=0,
\end{aligned}
$$


with $\mathbf{k}_{\mu \nu}$ and $\mathbf{K}_{\mu \nu}$ the coefficients of $\mathbf{K}$. Notice that by $\mathbf{k}_{\mu \nu}(0, b, B)=0$ and $\mathbf{K}_{\mu \nu}(0, b, B)=0$, for $\varrho=0$ there is a unique solution $(b, B) \in X_{2 k_{1}}$ given by

$$
b_{\mu \nu}=\frac{\mathrm{i} k_{\mu \nu}(0)}{\lambda(0) \cdot(\mu-\nu)}, \quad B_{\mu \nu}(0)=-\mathrm{i} R_{\mathcal{H}}(\lambda(0) \cdot(\mu-\nu)) K_{\mu \nu}(0) .
$$

Notice that for $i=0,1$ we have $R_{\mathcal{H}}(\zeta) P_{c}\left(\omega_{0}\right) \circ \partial_{a}^{i} \in B\left(\Sigma_{2 l}, \Sigma_{2 l}\right)$ for any $l \in \mathbb{Z}$, $\zeta \notin \sigma_{e}(\mathcal{H})$ and $a \in\{1,2,3\}$. The case $i=0$ can be proved by induction over $l$ using material in Section 7 The case $i=1$ holds for $\mathcal{H}_{0}:=\sigma_{2}(-\Delta+\omega)$. Finally, these facts and the resolvent identity yield the case $i=1$ for $\mathcal{H}$. Then Lemma 10.2 is a consequence of the implicit function theorem.

Substituting $\varrho=\varrho(f)$ we obtain what follows:

Lemma 10.3. Set $K_{1}=K(\varrho(f)), \widetilde{K}_{1}=\widetilde{K}(\varrho(f), b(\varrho(f)), B(\varrho(f)))$ and $\chi_{1}=$ $\chi(\varrho(f))$. Then we have

$$
\left\{\chi_{1}, H_{2}\right\}=K_{1}+\widetilde{K}_{1}+Z_{1}+L_{1},
$$

where $Z_{1}$ is in normal form and homogeneous of degree $M_{0}+1$ in $(z, \bar{z}, f)$ and

$$
L_{1}=\left\langle V_{j}(\varrho(f)) f \mid f\right\rangle \widetilde{\chi}_{j}+\left\langle T_{j} f \mid f\right\rangle \widehat{\chi}_{j},
$$

where: $V_{j}(\varrho) \in C^{k_{0}-1}\left(U, \Sigma_{2 k_{1}-1}\right), T_{j} \in B\left(\Sigma_{-2 k_{1}}, \Sigma_{l}\right)$ for all $l ; \tilde{\chi}_{j}$ and $\hat{\chi}_{j}$ are polynomials like $\chi_{1}$, with monomials of no smaller degree and with coefficients in $C^{k_{0}-1}$ in $\varrho$.

Proof. By direct computation (10.11) holds with

$$
\begin{aligned}
& L_{1}=\mathrm{i}\left\langle\beta^{\prime}\left(\phi^{2}\right) \phi^{2} \sigma_{3} f \mid f\right\rangle \partial_{Q(f)} \chi_{1}+\frac{1}{2}\left\langle\sigma_{3}\left(\partial_{a} V_{\omega_{0}}\right) f \mid \sigma_{1} f\right\rangle \partial_{\Pi_{a}(f)} \chi_{1} \\
& +z^{\mu} \bar{z}^{\nu} \partial_{\varrho_{i}} a_{\mu \nu}^{(\ell)}\left(\partial_{\varrho_{j}} \chi_{1}\left\{\varrho_{i}(f), \varrho_{j}(f)\right\}+z^{\mu^{\prime}} \bar{z}^{\nu^{\prime}}\left\langle\sigma_{1} \sigma_{3} B_{\mu^{\prime} \nu^{\prime}},\left\{\varrho_{i}(f), f\right\}\right\rangle\right),
\end{aligned}
$$

with $\varrho_{0}(f)=Q(f)$ and $\varrho_{a}(f)=\Pi_{a}(f)$ for $a=1,2,3$. We have

$$
\begin{aligned}
& \left\{Q(f), \Pi_{a}(f)\right\}=\mathrm{i}\left\langle\sigma_{1} f \mid P_{c}\left(\omega_{0}\right) \partial_{a} f\right\rangle=\mathrm{i}\left\langle\sigma_{1} f \mid \partial_{a} P_{d}\left(\omega_{0}\right) f\right\rangle, \\
& \left\langle\sigma_{1} \sigma_{3} B_{\mu \nu} \mid\{Q(f), f\}\right\rangle=\left\langle B_{\mu \nu} \mid P_{c}\left(\omega_{0}\right) \sigma_{3} f\right\rangle, \\
& \left\{\Pi_{b}(f), \Pi_{a}(f)\right\}=\mathrm{i}\left\langle\sigma_{1} \sigma_{3} \partial_{b} f \mid P_{c}\left(\omega_{0}\right) \partial_{a} f\right\rangle=-\mathrm{i}\left\langle\sigma_{1} \sigma_{3} \partial_{b} f \mid P_{d}\left(\omega_{0}\right) \partial_{a} f\right\rangle, \\
& \left\langle\sigma_{1} \sigma_{3} B_{\mu \nu} \mid\left\{\Pi_{a}(f), f\right\}\right\rangle=\left\langle B_{\mu \nu} \mid P_{c}\left(\omega_{0}\right) \partial_{a} f\right\rangle \\
& =-\left\langle\partial_{a} B_{\mu \nu} \mid f\right\rangle-\left\langle B_{\mu \nu} \mid P_{d}\left(\omega_{0}\right) \partial_{a} f\right\rangle .
\end{aligned}
$$

(10.18)-(10.19) yield the properties of $L_{1}$.

\section{Canonical transformations}

We consider the functions $\chi$ :

$$
\chi=\sum_{|\mu+\nu|=M_{0}+1} b_{\mu \nu}(\varrho(f)) z^{\mu} \bar{z}^{\nu}+\sum_{|\mu+\nu|=M_{0}} z^{\mu} \bar{z}^{\nu}\left\langle\sigma_{1} \sigma_{3} B_{\mu \nu}(\varrho(f)) \mid f\right\rangle .
$$

We assume $b_{\mu \nu} \in C^{k_{0}}\left(\mathbb{R}^{4}, \mathbb{C}\right)$ and $B_{\mu \nu} \in C^{k_{0}}\left(\mathbb{R}^{4}, \Sigma_{4 k_{1}}\left(\mathbb{R}^{3}, \mathbb{C}^{2}\right)\right)$ satisfying the symmetries in (4.12). Here $k_{0}$ and $k_{1}$ are fixed, very large and in $\mathbb{N}$ and $k_{1} \gg k_{0}$. We will always assume $B_{\mu \nu} \in L_{c}^{2}\left(\mathcal{H}_{\omega_{0}}\right)$. We want to consider the flow $\phi^{t}$ associated to the Hamiltonian vector field $X_{\chi}$ at time $t=1$ and use it to change coordinates.

Our first step consists in setting up the Hamiltonian system associated to $\chi$. It is a quasilinear symmetric hyperbolic system. 
Lemma 11.1. Consider $\chi$ as in (11.1) satisfying the symmetries in (4.12). Summing on repeated indexes, the following holds:

$$
\{f, \chi\}=\mathcal{L} f+\mathcal{D}, \quad\left\{z_{j}, \chi\right\}=Z_{j}, \quad \mathcal{L}:=\mathcal{A}_{a} \partial_{a}+\mathcal{C} \sigma_{3},
$$

where the coefficients are given by the following formulas:

$$
\begin{aligned}
& \mathcal{A}_{a}=\partial_{\Pi_{a}(f)} \chi, \quad \mathcal{C}=-\mathrm{i} \partial_{Q(f)} \chi, \quad Z_{j}=-\mathrm{i} \partial_{\bar{z}_{j}} \chi, \\
& \mathcal{D}=-\mathrm{i} z^{\mu} \bar{z}^{\nu} B_{\mu \nu}(\varrho(f))-P_{d}\left(\omega_{0}\right) \mathcal{L} f .
\end{aligned}
$$

The coefficients can be thought of as dependent on $(z, f, \varrho(f))$. If we substitute $\varrho(f)$ with an independent variable $\varrho \in \mathbb{R}^{4}$, then we have $\mathcal{A}_{a} \in C^{k_{0}-1}(\mathfrak{V}, \mathbb{R}), \mathrm{i} \mathcal{B}_{a}, \mathrm{iC} \in$ $C^{k_{0}-1}(\mathfrak{V}, \mathbb{R})$ and $\mathcal{D} \in C^{k_{0}-1}\left(\mathfrak{V}, \Sigma_{4 k_{1}}\right)$, with $\mathfrak{V}$ a neighborhood of the origin in $\mathbb{C}^{m} \times \Sigma_{8 k_{0}-4 k_{1}} \times \mathbb{R}^{4}$.

The following inequalities hold:

$$
\begin{aligned}
& |\mathcal{A}|+|\mathcal{C}| \leq C|z|^{M_{0}}\left(|z|+\|f\|_{\Sigma_{-4 k_{1}}}\right), \\
& |Z|+\|\mathcal{D}\|_{\Sigma_{4 k_{1}}} \leq C|z|^{M_{0}-1}\left(|z|+\|f\|_{\Sigma_{-4 k_{1}}}\right) .
\end{aligned}
$$

Proof. (11.2)-111.3) follow from a simple computation. The rest follows by Subsection 8.3 for $n_{0}=k_{1}$ and $n=k_{0}$.

Lemma 11.1 assures us that we are within the framework of Section 8 and that the Lie transform $\phi=\phi^{1}$ associated to the following system is well defined:

$$
\dot{f}=\{f, \chi\}, \quad \dot{z}=\{z, \chi\} .
$$

In particular, we have:

Lemma 11.2. Suppose $k_{1}$ is sufficiently large. Set $\left(z^{t}, f^{t}\right)=\phi^{t}(z, f)$. Then we have

$$
z^{t}=z+\mathcal{Z}_{t}, \quad f^{t}=e^{\sigma_{3} \gamma_{t}} \tau_{\mathbf{A}_{t}} f+\mathcal{G}_{t}
$$

with $\tau_{\mathbf{A}_{t}} f(x)=f\left(x-\mathbf{A}_{t}\right), \mathbf{A}_{t}=-\int_{0}^{t} \mathcal{A}(\tau) d \tau, \mathcal{Z}_{t}=\int_{0}^{t} Z(\tau) d \tau, \gamma_{t}=\int_{0}^{t} \mathcal{C}(s) d s$ and

$$
\mathcal{G}_{t}(x)=\int_{0}^{t} e^{-\sigma_{3} \int_{s}^{t} \mathcal{C}(\tau) d \tau} \mathcal{D}\left(s, x+\int_{s}^{t} \mathcal{A}(\tau) d \tau\right) d s .
$$

We have $\boldsymbol{A}_{t}=\boldsymbol{A}_{t}(z, f, \varrho(f))$, and $\gamma_{t}=\gamma_{t}(z, f, \varrho(f))$, with $C^{k_{0}-1}$ dependence in $z \in$ $\mathbb{C}^{m}, f \in \Sigma_{8 k_{0}-4 k_{1}}$ and $\varrho \in \mathbb{R}^{4}$. The same statement holds for $\mathcal{Z}_{t}=\mathcal{Z}_{t}(z, f, \varrho(f))$, resp. $\mathcal{G}_{t}=\mathcal{G}_{t}(z, f, \varrho(f))$, with values in $\mathbb{C}^{m}$, resp. $\Sigma_{4 k_{1}-k_{0}}$.

The $f^{t}$ has $C^{k_{0}-1}$ dependence in $z \in \mathbb{C}^{m}, f \in \Sigma_{8 k_{0}-4 k_{1}}$ and $\varrho \in \mathbb{R}^{4}$ with values in $\Sigma_{9 k_{0}-4 k_{1}}$.

The $\varrho\left(f^{t}\right)$ has $C^{k_{0}-1}$ dependence in $z \in \mathbb{C}^{m}, f \in \Sigma_{8 k_{0}-4 k_{1}}$ and $\varrho(f) \in \mathbb{R}^{4}$ with values in $\mathbb{R}^{4}$.

There is a fixed constant $C$ such that

$$
\begin{gathered}
\left|\mathcal{Z}_{t}\right|+\left\|\mathcal{G}_{t}\right\|_{\Sigma_{4 k_{1}}} \leq C|z|^{M_{0}-1}\left(|z|+\|f\|_{\Sigma_{-4 k_{1}}}\right), \\
\left|\mathbf{A}_{t}\right|+\left|\gamma_{t}\right| \leq C|z|^{M_{0}-1}\left(|z|+\|f\|_{\Sigma_{-4 k_{1}}}\right)^{2} .
\end{gathered}
$$

Proof. This is the analogue of Lemmas 8.4 and 8.6

We will set $\phi=\phi^{1},\left(z^{\prime}, f^{\prime}\right)=\phi(z, f)$ and we will drop the subindex $t$ for $t=1$, that is, $\mathbf{A}=\mathbf{A}_{t}$, etc. 
Lemma 11.3. In the above notation we have

$$
\begin{gathered}
\left|Q\left(f^{\prime}\right)-Q(f)\right| \leq C|z|^{M_{0}-1}\left(|z|+\|f\|_{\Sigma_{-4 k_{1}}}\right)^{2}, \\
\left|\Pi\left(f^{\prime}\right)-\Pi(f)\right| \leq C|z|^{M_{0}-1}\left(|z|+\|f\|_{\Sigma_{-4 k_{1}+1}}\right)^{2}
\end{gathered}
$$

for a fixed $C$ dependent on $\left\|\left(b_{\mu \nu}, B_{\mu \nu}\right)\right\|_{C^{1}\left(\mathrm{U}_{4}\right)}$ with $\mathrm{U}_{4} \subset \mathbb{R}^{4}$ a preassigned neighborhood of the origin.

Proof. We have

$$
Q\left(f^{\prime}\right)=Q(f)+\int_{0}^{1}\{Q(f), \chi\} \circ \phi^{t} d t
$$

with

$$
\{Q(f), \chi\}=\left\{Q(f), \Pi_{a}(f)\right\} \partial_{\Pi_{a}(f)} \chi+z^{\mu} \bar{z}^{\nu}\left\langle\sigma_{1} \sigma_{3} B_{\mu \nu}(\varrho(f)) \mid\{Q(f), f\}\right\rangle .
$$

We have $|\{Q(f), \chi\}| \leq C\left(|b|+\|B\|_{\Sigma_{4 k_{1}}}\right)|z|^{M_{0}-1}\left(|z|+\|f\|_{\Sigma_{-4 k_{1}}}\right)^{2}$ for a fixed $C$ dependent on $\left\|\left(b_{\mu \nu}, B_{\mu \nu}\right)\right\|_{C^{1}}$ by the formulas in (10.19). The integral in (11.11) has the same upper bound by Lemma 11.2, in particular by (11.6) and inequalities (11.7) -(11.8). This proves (11.9). For $\Pi_{a}\left(f^{\prime}\right)$ we have a similar argument by the following formulas and estimates:

$$
\left\{\Pi_{b}(f), \Pi_{a}(f)\right\}=\mathrm{i}\left\langle\sigma_{1} \sigma_{3} \partial_{b} f \mid P_{c}\left(\omega_{0}\right) \partial_{a} f\right\rangle=-\mathrm{i}\left\langle\sigma_{1} \sigma_{3} \partial_{b} f \mid P_{d}\left(\omega_{0}\right) \partial_{a} f\right\rangle ;
$$

$\left|\left\langle\sigma_{1} \sigma_{3} \partial_{b} f \mid P_{d}\left(\omega_{0}\right) \partial_{a} f\right\rangle\right| \leq C_{\ell}\|f\|_{\Sigma_{-\ell}}^{2}$ and $\left|\left\langle B_{\mu \nu} \mid P_{d}\left(\omega_{0}\right) \partial_{a} f\right\rangle\right| \leq C_{\ell}\|B\|_{\Sigma_{-\ell}}\|f\|_{\Sigma_{-\ell}}$ for any $\ell$. We have $\left|\left\langle\partial_{a} B_{\mu \nu} \mid f\right\rangle\right| \leq C\|B\|_{\Sigma_{4 k_{1}}}\|f\|_{\Sigma_{-4 k_{1}+1}}$ by $\left\|\partial_{a} B\right\|_{\Sigma_{4 k_{1}-1}} \leq c\|B\|_{\Sigma_{4 k_{1}}}$; see Lemma 7.5. Thanks to (10.19) and Lemma 11.2 these inequalities yield (11.10).

The information in Lemma 11.2 is not sufficiently precise for our purposes. Let us consider for any fixed $(z(0), f(0))$ the system

$$
\begin{aligned}
& \dot{g}=-\mathrm{i} \zeta^{\mu} \zeta^{\nu} B_{\mu \nu}(\varrho(f(0))), \\
& \dot{\zeta}_{j}=-\mathrm{i} \nu_{j} \frac{\zeta^{\mu} \zeta^{\nu}}{\bar{\zeta}_{j}}\left(b_{\mu \nu}(\varrho(f(0)))+\left\langle\sigma_{1} \sigma_{3} B_{\mu \nu}(\varrho(f(0))) \mid f\right\rangle\right), \\
& g(0)=f(0), \quad \zeta(0)=z(0) .
\end{aligned}
$$

Notice that wellposedness, regularity of the flow, and smooth dependence on the coefficients $(b(\varrho(f(0)))), B(\varrho(f(0)))$ fall within the scope of the theory of ordinary equations. Denote $\phi_{0}^{t}$ the flow of (11.12). In particular for $\left(\zeta^{t}, g^{t}\right)=\phi_{0}^{t}(\zeta, g)$ we have

$$
\begin{aligned}
\zeta^{t} & =\zeta+\mathbf{Z}_{t}(\zeta, g, b(\varrho(f)), B(\varrho(f))), \\
g^{t} & =g+\mathbf{G}_{t}(\zeta, g, b(\varrho(f)), B(\varrho(f))),
\end{aligned}
$$

with $\mathbf{Z}_{t}(\zeta, g, b, B)$ (resp. $\mathbf{G}_{t}(\zeta, g, b, B)$ ) with $C^{\infty}$ dependence on $t, \zeta \in \mathbb{C}^{m}$ and $g \in \Sigma_{-4 k_{1}}$, and $(b, B)$, with values in $\mathbb{C}^{m}\left(\right.$ resp. $\left.\Sigma_{4 k_{1}}\left(\mathbb{R}^{3}, \mathbb{C}^{2}\right)\right)$ and with

$$
\mathbf{Z}_{t}(\zeta, g, 0,0) \equiv 0, \quad \mathbf{G}_{t}(\zeta, g, 0,0) \equiv 0 .
$$

Furthermore $\mathbf{Z}_{t}$, resp. $\mathbf{G}_{t}$, satisfy uniformly in $t$ the same bounds (11.7) of $\mathcal{Z}$, resp. $\mathcal{G}$.

We compare the solutions of (11.5) with those of (11.12). Denote $\left(z^{\prime}, f^{\prime}\right)=$ $\phi^{1}(z, f)$ and $\left(\zeta^{\prime}, g^{\prime}\right)=\phi_{0}^{1}(z, f)$. 
Lemma 11.4. For a $C$ as in Lemma 11.3 we have for any $j \leq k_{1}$,

$$
\left|z^{\prime}-\zeta^{\prime}\right|+\left\|f^{\prime}-g^{\prime}\right\|_{\Sigma_{-4 j-1}} \leq C\left(|z|+\|f\|_{\Sigma_{-4 j+1}}\right)^{M_{0}+1} .
$$

Proof. Set $\mathbf{M}(t)=|z(t)|+|\zeta(t)|+\|f(t)\|_{\Sigma_{1-4 j}}+\|g(t)\|_{\Sigma_{1-4 j}}$. We have

$$
\dot{f}-\dot{g}=\mathrm{i} \zeta^{\mu} \bar{\zeta}^{\nu} B_{\mu \nu}(\varrho(f(0)))-\mathrm{i} z^{\mu} \bar{z}^{\nu} B_{\mu \nu}(\varrho(f))-P_{d}\left(\omega_{0}\right) \mathcal{L} f+\left(\mathcal{A}_{a} \partial_{a}+\mathcal{C} \sigma_{3}\right) f .
$$

The rhs has $\Sigma_{-4 j}$ norm bounded by

$$
\begin{aligned}
& \left|\zeta^{\mu} \bar{\zeta}^{\nu}-z^{\mu} \bar{z}^{\nu}\right|\left\|B_{\mu \nu}(\varrho(f(0)))\right\|_{\Sigma_{-4 j}}+\left|z^{\mu} \bar{z}^{\nu}\right|\left\|B_{\mu \nu}(\varrho(f))-B_{\mu \nu}(\varrho(f(0)))\right\|_{\Sigma_{-4 j}} \\
& \quad+\left\|P_{d}\left(\omega_{0}\right) \mathcal{L} f\right\|_{\Sigma_{-4 j}}+\left\|\left(\mathcal{A}_{a} \partial_{a}+\mathcal{C} \sigma_{3}\right) f\right\|_{\Sigma_{-4 j} .}
\end{aligned}
$$

Then

$$
\|f(t)-g(t)\|_{\Sigma_{-4 j}} \leq C \int_{0}^{t} \mathbf{M}^{M_{0}-1}(t)|z(\tau)-\zeta(\tau)| d \tau+C \int_{0}^{t} \mathbf{M}^{M_{0}+2}(t) d \tau .
$$

Similarly

$$
\begin{aligned}
& |z(t)-\zeta(t)| \leq C \int_{0}^{t} \mathbf{M}^{M_{0}-1}(t)\left(|z(\tau)-\zeta(\tau)|+\|f(\tau)-g(\tau)\|_{\Sigma_{-4 j}}\right) d \tau \\
& +C \int_{0}^{t} \mathbf{M}^{M_{0}+1}(t) d \tau .
\end{aligned}
$$

Then the statement follows from Gronwall's inequality since $\mathbf{M}(t) \leq C \mathbf{M}(0)$. For instance, $|z(t)|+\|f(t)\|_{\Sigma_{1-4 j}} \leq C\left(|z(0)|+\|f(0)\|_{\Sigma_{1-4 j}}\right.$ ) follows by formula (11.6) and by inequalities (11.7)-(11.8).

Lemma 11.5. In the above notation of Lemma 11.5 we have

$$
\begin{gathered}
\left|Q\left(f^{\prime}\right)-Q\left(g^{\prime}\right)\right| \leq C\left(|z|+\|f\|_{\Sigma_{-4 k_{1}+1}}\right)^{M_{0}+2}, \\
\left|\Pi\left(f^{\prime}\right)-\Pi\left(g^{\prime}\right)\right| \leq C\left(|z|+\|f\|_{\Sigma_{-4 k_{1}+3}}\right)^{M_{0}+2} .
\end{gathered}
$$

Proof. We have

$$
\begin{aligned}
& \frac{d}{d t}\left(\Pi_{b}(f)-\Pi_{b}(g)\right)=\mathrm{i} \zeta^{\mu} \bar{\zeta}^{\nu}\left\langle B_{\mu \nu}(\varrho(f(0))) \mid \sigma_{3} \sigma_{1} \partial_{b} g\right\rangle \\
& +\mathrm{i} z^{\mu} \bar{z}^{\nu}\left\langle B_{\mu \nu}(\varrho(f)) \mid \sigma_{3} \sigma_{1} \partial_{b} f\right\rangle-\left\langle P_{d}\left(\omega_{0}\right) \mathcal{L} f \mid \sigma_{3} \sigma_{1} \partial_{b} f\right\rangle .
\end{aligned}
$$

The rhs can be bounded above by the rhs of (11.17) computed at time $t$. Integrating, using the fact that $(z, f)=(\zeta, g)$ at $t=0$ and by (11.6) and (11.7)-(11.8) we get (11.17). The proof of (11.16) is similar.

\section{BIRKHOFF NORMAL FORMS}

Our goal in this section is to prove the following result.

Theorem 12.1. For any integer $2 \leq \ell \leq 2 N+1$ there are a $\delta_{0}>0$ and $M \gg N$ large such that in the subset of $\Sigma_{4 M}$ defined by $|z|+\|f\|_{H^{1}}<\delta_{0}$ is defined a canonical transformation $\mathcal{T}_{r}$ which is differentiable as a map with values in $\Sigma_{1}$ and whose image contains a similar subset of $\Sigma_{4 M}$ defined by $|z|+\|f\|_{H^{1}}<\delta_{0}^{\prime}$, s.t.

$$
H^{(\ell)}:=K \circ \mathcal{T}_{\ell}=\psi(\varrho(f))+H_{2}^{(\ell)}+Z^{(\ell)}+\mathcal{R}^{(\ell)},
$$


with $\psi(\varrho(f))$ the same as (9.5) and where:

(i) $H_{2}^{(\ell)}=H_{2}^{(2)}$ for $\ell \geq 2$, is of the form (4.9) where $a_{\mu \nu}^{(\ell)}$ satisfy (9.8)-(9.9);

(ii) $Z^{(\ell)}$ is in normal form, with monomials of degree $\leq \ell$ whose coefficients satisfy (4.12);

(iii) we have $\mathcal{T}_{\ell}=\phi_{\ell} \circ \ldots \circ \phi_{1}$, with each $\phi_{j}$ a Lie transformation associated to a function (11.1) with $M_{0}=j$;

(iv) we have $\mathcal{R}^{(\ell)}=\sum_{d=0}^{6} \mathcal{R}_{d}^{(\ell)}$ with the following properties (for $k_{2}(\ell) \ll$ $k_{3}(\ell) \ll M$ pairs of appropriate large numbers with $k_{j}(\ell+1) \ll k_{j}(\ell)$ for all $\ell$ and for $j=2,3)$ :

(iv.0) we have with $\left|\partial_{\varrho}^{l} a_{\mu \nu}^{(\ell)}(\varrho)\right| \leq C_{l}$ for $|l| \leq k_{2}(\ell)$,

$$
\mathcal{R}_{0}^{(\ell)}=\sum_{|\mu+\nu|=\ell+1} z^{\mu} \bar{z}^{\nu} a_{\mu \nu}^{(\ell)}(\varrho(f))
$$

(iv.1) we have with $\left\|\partial_{\varrho}^{l} G_{\mu \nu}^{(\ell)}(\varrho)(\cdot)\right\|_{\Sigma_{4 k_{3}(\ell)}\left(\mathbb{R}^{3}, \mathbb{C}^{2}\right)} \leq C_{l}$ for $|l| \leq k_{2}(\ell)$,

$$
\mathcal{R}_{1}^{(\ell)}=\sum_{|\mu+\nu|=\ell} z^{\mu} \bar{z}^{\nu}\left\langle\sigma_{1} \sigma_{3} G_{\mu \nu}^{(\ell)}(\varrho(f)) \mid f\right\rangle \text { with }
$$

(iv.2-5) in $\mathcal{U}$ we have for $2 \leq d \leq 5$ and for $\eta^{T}=(\zeta, \bar{\zeta})$ with $\zeta \in \mathbb{C}$,

$$
\mathcal{R}_{d}^{(\ell)}=\int_{\mathbb{R}^{3}} F_{d}^{(\ell)}(x, z, f, f(x), \varrho(f)) f^{d}(x) d x+\widehat{\mathcal{R}}_{d}^{(\ell)},
$$

with for $|l| \leq k_{2}(\ell)$

$$
\begin{gathered}
\left\|\partial_{z, \bar{z}, \zeta, \bar{\zeta}, f, \varrho}^{l} F_{d}^{(\ell)}(\cdot, z, f, \eta, \varrho)\right\|_{\Sigma_{4 k_{3}(\ell)}\left(\mathbb{R}^{3}, B\left(\left(\mathbb{C}^{2}\right) \otimes d, \mathbb{C}\right)\right.} \leq C_{l}, \\
\text { with } F_{2}^{(\ell)}(x, 0,0,0,0)=0 \text { and with } \widetilde{\mathcal{R}}_{d}^{(\ell)}(z, f, \varrho(f)) \text { s.t. } \\
\widehat{\mathcal{R}}_{d}^{(\ell)} \in C^{k_{2}(\ell)}(\mathcal{U} \times \mathbb{R}, \mathbb{R}), \quad\left|\widehat{\mathcal{R}}_{d}^{(\ell)}(z, f, \varrho)\right| \leq C\|f\|_{\Sigma_{-4 k_{3}(\ell)}}^{d}, \\
\left|\widehat{\mathcal{R}}_{2}^{(\ell)}(z, f, \varrho)\right| \leq C\left(|z|+|\varrho|+\|f\|_{\Sigma_{-4 k_{3}(\ell)}}\right)\|f\|_{\Sigma_{-4 k_{3}(\ell)}}^{2} ;
\end{gathered}
$$

(iv.6) $\mathcal{R}_{6}^{(\ell)}=\int_{\mathbb{R}^{3}} B\left(|f(x)|^{2} / 2\right) d x$.

12.1. Pullback of multilinear forms. The method of Birkhoff normal forms is implemented using the flows of auxiliary Hamiltonians $\chi$ like in (11.1). In particular, we will assume for the moment that the degree is $M_{0}+1$ and that $b_{\mu \nu} \in C^{k_{0}}\left(\mathbb{R}^{4}, \mathbb{C}\right)$ and $B_{\mu \nu} \in C^{k_{0}}\left(\mathbb{R}^{4}, \Sigma_{4 k_{1}}\left(\mathbb{R}^{3}, \mathbb{C}^{2}\right)\right)$. In the proof of Theorem 12.1, $\chi$ needs to solve a homological equation. In this section we look at pullbacks of the various terms of the Hamiltonian by means of the Lie transform associated to $\chi$. In general these terms are pulled back into other remainder terms which are less regular. By this we mean both that their coefficients are less than $C^{k_{0}}$ and with values in some $\Sigma_{4 k}$ with $k<k_{1}$. In general this loss of regularity is harmless. However we have to make sure that the terms which enter in the homological equation of $\chi$, which is used to find a useful $\chi$, have the same regularity as $\chi$. It is at this stage that we use the associated simplified system (11.12). We will now consider a number of technical lemmas. 
Lemma 12.2. Let $F=F(z, f, \varrho)$ be $C^{k_{0}}$ in $z \in \mathbb{C}^{m}, f \in \Sigma_{-4 k_{1}}$ and $\varrho \in \mathbb{R}^{4}$ with values in $\Sigma_{4 k_{1}}\left(\mathbb{R}^{3}, B^{2}(\mathbb{C}, \mathbb{C})\right)$. For $M_{0}=1$ we assume $F(0,0,0)=0$. Then

$$
\begin{aligned}
& \left\langle F\left(z^{\prime}, f^{\prime}, \varrho\left(f^{\prime}\right)\right) \mid \mathcal{G}^{2}\right\rangle=\sum_{|\mu+\nu|=M_{0}+1} k_{\mu \nu}(\varrho(f), b(\varrho(f)), B(\varrho(f))) z^{\mu} \bar{z}^{\nu} \\
& +\sum_{|\mu+\nu|=M_{0}} z^{\mu} \bar{z}^{\nu}\left\langle\sigma_{1} \sigma_{3} K_{\mu \nu}(\varrho(f), b(\varrho(f)), B(\varrho(f))) \mid f\right\rangle+\boldsymbol{R},
\end{aligned}
$$

where the following holds:

(i) $k_{\mu \nu}(0, b, B)=0$ and $K_{\mu \nu}(0, b, B)=0 ; k_{\mu \nu}(\varrho, b, B) \in \mathbb{C}$ and $K_{\mu \nu}(\varrho, b, B) \in$ $\Sigma_{4 k_{1}}$ are $C^{k_{0}-1}$ in $\varrho$, in $b_{\mu \nu} \in \mathbb{C}$ and in $B_{\mu \nu} \in \Sigma_{4 k_{1}}$.

(ii) $\boldsymbol{R}$ is a sum of terms of the form $\mathcal{R}^{\left(M_{0}+1\right)}$, that is, as in the statement of Theorem 12.1, with $k_{3}=k_{1}-2 k_{0}$ and $k_{2}=k_{0}-M_{0}-3$.

If $M_{0}>1$ formula (12.4) holds with only $\boldsymbol{R}$ on the rhs.

Proof. We have for $\varrho=\varrho(f)$ and $\varrho^{\prime}=\varrho\left(f^{\prime}\right)$ and $\delta \varrho=\varrho^{\prime}-\varrho$

$$
\left\langle F\left(z^{\prime}, f^{\prime}, \varrho^{\prime}\right) \mid \mathcal{G}^{2}\right\rangle=\left\langle F\left(z^{\prime}, f^{\prime}, \varrho\right) \mid \mathcal{G}^{2}\right\rangle+\int_{0}^{1}\left\langle\partial_{\varrho} F\left(z^{\prime}, f^{\prime}, \varrho+t \delta \varrho\right) \mid \mathcal{G}^{2}\right\rangle d t \cdot \delta \varrho .
$$

By Lemma 11.2 the second term on the rhs is $C^{k_{0}-1}$ in $z \in \mathbb{C}^{m}, f \in \Sigma_{8 k_{0}-4 k_{1}}$ and $\varrho \in \mathbb{R}^{4}$. Furthermore $\delta \varrho$ satisfies $|\delta \varrho| \leq C|z|^{M_{0}-1}\left(|z|+\|f\|_{\Sigma_{-4 k_{1}+1}}\right)^{2}$ by Lemma 11.3. By $k_{0}=k_{2}+M_{0}+3$ we can write the second term on the rhs of (12.5) as $\mathcal{R}_{0}^{\left(M_{0}+1\right)}+\mathcal{R}_{1}^{\left(M_{0}+1\right)}+\widehat{\mathcal{R}}_{2}^{\left(M_{0}+1\right)}$, just by performing an appropriate and partial Taylor expansion. If $M_{0}>1$, the same result holds for the first term on the rhs of (12.5). Now let $M_{0}=1$. By Lemmas 11.2 and 8.7 it is $C^{k_{0}-1}$ in $z \in \mathbb{C}^{m}, f \in \Sigma_{8 k_{0}-4 k_{1}}$ and $\varrho \in \mathbb{R}^{4}$ and can be expressed as $\left\langle F(0,0, \varrho) \mid \mathcal{G}^{2}\right\rangle$ plus a term which is like the above ones and can be absorbed in $\mathbf{R}$. Writing $F(\varrho)=F(0,0, \varrho)$, we have succinctly

$$
\left\langle F(\varrho) \mid \mathcal{G}^{2}\right\rangle=\left\langle F(\varrho) \mid \mathbf{G}_{1}^{2}\right\rangle-2\left\langle F(\varrho) \mid\left(\mathbf{G}_{1}-\mathcal{G}\right) \mathbf{G}_{1}\right\rangle+\left\langle F(\varrho) \mid\left(\mathbf{G}_{1}-\mathcal{G}\right)^{2}\right\rangle,
$$

with $\mathbf{G}_{1}$ from the associated system; see (11.13). By Lemma 11.4 the rhs is $C^{k_{0}-1}$ in $z \in \mathbb{C}^{m}, f \in \Sigma_{8 k_{0}-4 k_{1}}$ and $\varrho \in \mathbb{R}^{4}$. We have

$$
\left\langle F(\varrho) \mid\left(\mathbf{G}_{1}-\mathcal{G}\right) \mathbf{G}_{1}\right\rangle=\left\langle F(\varrho) \mid\left(g^{\prime}-f^{\prime}\right) \mathbf{G}_{1}\right\rangle+\left\langle F(\varrho) \mid\left(e^{\sigma_{3} \gamma} \tau_{\mathbf{A}} f-f\right) \mathbf{G}_{1}\right\rangle .
$$

Then

$$
\begin{aligned}
& \left|\left\langle F(\varrho) \mid\left(g^{\prime}-f^{\prime}\right) \mathbf{G}_{1}\right\rangle\right| \leq\|F\|_{\Sigma_{4 k_{1}}}\left\|\mathbf{G}_{1}\right\|_{\Sigma_{4 k_{1}}}\left\|g^{\prime}-f^{\prime}\right\|_{\Sigma_{4 k_{1}}} \\
& \leq C\|F\|_{\Sigma_{4 k_{1}}}\left(|z|+\|f\|_{\Sigma_{1-4 k_{1}}}\right)^{3} .
\end{aligned}
$$

Similarly

$$
\begin{aligned}
& \left|\left\langle F(\varrho) \mid\left(e^{\sigma_{3} \gamma} \tau_{\mathbf{A}} f-f\right) \mathbf{G}_{1}\right\rangle\right| \leq\|F\|_{\Sigma_{4 k_{1}}}\left\|\mathbf{G}_{1}\right\|_{\Sigma_{4 k_{1}}}\left\|\left(e^{\sigma_{3} \gamma} \tau_{\mathbf{A}} f-f\right)\right\|_{\Sigma_{4 k_{1}}} \\
& \leq C\|F\|_{\Sigma_{4 k_{1}}}\left(|z|+\|f\|_{\Sigma_{1-4 k_{1}}}\right)^{3} .
\end{aligned}
$$

Hence the second term on the rhs of (12.6) can be absorbed in $\mathbf{R}$. Similar reasoning applies to the third term on the rhs of (12.6). We finally show that the first term on the rhs of (12.6) yields the first two terms on the rhs of (12.4) plus a term which can be absorbed in $\mathbf{R}$. We know that $\mathbf{G}_{1}=\mathbf{G}_{1}\left(z, f, b_{\mu \nu}, B_{\mu \nu}\right)$ is $C^{\infty}$ in 
$z \in \mathbb{C}^{m}, f \in \Sigma_{-4 k_{1}}$ and $\left(b_{\mu \nu}, B_{\mu \nu}\right)$, with values in $\Sigma_{4 k_{1}}\left(\mathbb{R}^{3}, \mathbb{C}^{2}\right)$ with $\left\|\mathbf{G}_{1}\right\|_{\Sigma_{4 k_{1}}}=$ $O\left(|z|+\|f\|_{\Sigma_{-4 k_{1}}}\right)$. We can consider

$$
\begin{aligned}
& \left\langle F(\varrho) \mid \mathbf{G}_{1}^{2}\right\rangle=\sum_{|\mu+\nu|=2} h_{\mu \nu}(\varrho, b, B) z^{\mu} \bar{z}^{\nu} \\
& +\sum_{|\mu+\nu|=1} z^{\mu} \bar{z}^{\nu}\left\langle\sigma_{1} \sigma_{3} H_{\mu \nu}(\varrho, b, B) \mid f\right\rangle+\widetilde{\mathcal{R}}
\end{aligned}
$$

with $\widetilde{\mathcal{R}}=O\left(|z|+\|f\|_{\Sigma_{-4 k_{1}}}\right)^{3}$ and $C^{k_{0}}$ in $z, f, \varrho$, and with

$$
\begin{aligned}
& h_{\mu \nu}(\varrho, b, B):=\frac{1}{\mu ! \nu !} \partial_{z}^{\mu} \partial_{\bar{z}}^{\nu}\left\langle F(\varrho) \mid \mathbf{G}_{1}^{2}\right\rangle_{\mid(0,0, \varrho)}, \\
& H_{\mu \nu}(\varrho, b, B):=\sigma_{3} \sigma_{1} \partial_{z}^{\mu} \partial_{\bar{z}}^{\nu} \nabla_{f}\left\langle F(\varrho) \mid \mathbf{G}_{1}^{2}\right\rangle_{\mid(0,0, \varrho)} .
\end{aligned}
$$

$\widetilde{\mathcal{R}}$ can be absorbed in $\mathbf{R}$. The polynomial in (12.7) is like the one in the statement because of the hypothesis $F(\varrho)=F(0,0, \varrho)=0$ when $\varrho=0$ if $M_{0}=1$.

Lemma 12.3. Let $F=F(z, f, \varrho)$ with the same properties as in Lemma 12.2, Then, for a rhs which satisfies the same properties stated in Lemma 12.2 but with $k_{3}=k_{1}-3 k_{0}$ and $k_{2}=k_{0}-M_{0}-4$,

$$
\begin{aligned}
& \left\langle F\left(z^{\prime}, f^{\prime}, \varrho\left(f^{\prime}\right)\right) \mid \mathcal{G} f^{\prime}\right\rangle=\sum_{|\mu+\nu|=M_{0}+1} k_{\mu \nu}(\varrho(f), b(\varrho(f)), B(\varrho(f))) z^{\mu} \bar{z}^{\nu} \\
& +\sum_{|\mu+\nu|=M_{0}} z^{\mu} \bar{z}^{\nu}\left\langle\sigma_{1} \sigma_{3} K_{\mu \nu}(\varrho(f), b(\varrho(f)), B(\varrho(f))) \mid f\right\rangle+\boldsymbol{R} .
\end{aligned}
$$

Proof. We have

$$
\begin{aligned}
& \left\langle F\left(z^{\prime}, f^{\prime}, \varrho\left(f^{\prime}\right)\right) \mid \mathcal{G} f^{\prime}\right\rangle=\left\langle F\left(z^{\prime}, f^{\prime}, \varrho\left(f^{\prime}\right)\right) \mid \mathcal{G} f\right\rangle \\
& +\left\langle F\left(z^{\prime}, f^{\prime}, \varrho\left(f^{\prime}\right)\right) \mid \mathcal{G}\left(e^{\sigma_{3} \gamma} \tau_{\mathbf{A}}-1\right) f\right\rangle+\left\langle F\left(z^{\prime}, f^{\prime}, \varrho\left(f^{\prime}\right)\right) \mid \mathcal{G}^{2}\right\rangle .
\end{aligned}
$$

The third term on the rhs is like Lemma 12.3. The second can be absorbed in $\mathbf{R}$ by (11.8). We focus on the first term on the rhs of (12.9). By (11.7) we have

$$
\begin{aligned}
& \mathcal{G}(x)=\int_{0}^{1} \mathcal{D}(s, x) d s \\
& \left.+\int_{0}^{1}\left(e^{-\sigma_{3} \int_{s}^{t} \mathcal{C}(\tau) d \tau}-1\right) \mathcal{D}(s, x)+\int_{s}^{t} \mathcal{A}(\tau) d \tau\right) d s \\
& +\int_{0}^{1}\left(\left(\mathcal{D}(s, x)+\int_{s}^{t} \mathcal{A}(\tau) d \tau\right)-\mathcal{D}(s, x)\right) d s .
\end{aligned}
$$

The last two lines are $C^{k_{0}-1}$ in $z \in \mathbb{C}^{m}, f \in \Sigma_{8 k_{0}-4 k_{1}}$ and $\varrho \in \mathbb{R}^{4}$ with values in $\Sigma_{4 k_{1}-4 k_{0}}$ where they have norm smaller than $C\left(|z|+\|f\|_{\Sigma_{8 k_{0}-4 k_{1}}}\right)^{3}$. This implies that when we substitute (12.10) in the first term on the rhs of (12.9), the last two lines of (12.10) can be absorbed in $\mathbf{R}$. When we substitute (11.3), the first term on the rhs of (12.10) is equal to what follows:

$$
-\mathrm{i} \int_{0}^{1}\left(z^{\mu} \bar{z}^{\nu} B_{\mu \nu}(\varrho(f))\right) \circ \phi^{t} d t-\int_{0}^{1} P_{d}\left(\omega_{0}\right)(\mathcal{L} f) \circ \phi^{t} d t .
$$

By Lemma 11.1 the second term in (12.11) is $C^{k_{0}-1}$ in $z \in \mathbb{C}^{m}, f \in \Sigma_{8 k_{0}-4 k_{1}}$ and $\varrho \in \mathbb{R}^{4}$ with values in $\Sigma_{l}$ for any $l$ and with norm $O\left(|z|+\|f\|_{\Sigma_{4 k_{0}-4 k_{1}}}\right)^{M_{0}+2}$. The 
corresponding terms in (12.9) can then be absorbed in $\mathbf{R}$. The first term in (12.11) can be written as

$$
-\mathrm{i} B_{\mu \nu}(\varrho(f)) \int_{0}^{1}\left(z^{\mu} \bar{z}^{\nu}\right) \circ \phi_{0}^{t} d t
$$

plus an error term which can then be absorbed in $\mathbf{R}$ since it is $C^{k_{0}-1}$ in $z \in$ $\mathbb{C}^{m}, f \in \Sigma_{8 k_{0}-4 k_{1}}$ and $\varrho \in \mathbb{R}^{4}$ with values in $\Sigma_{4 k_{1}}$ and with norm smaller than $C\left(|z|+\|f\|_{\Sigma_{8 k_{0}-4 k_{1}}}\right)^{M_{0}+2}$. By (11.13) we have that (12.12) is

$$
-\mathrm{i} B_{\mu \nu}(\varrho(f)) z^{\mu} \bar{z}^{\nu}-\mathrm{i} B_{\mu \nu}(\varrho(f)) F_{\mu \nu}(z, f, \varrho(f), b(\varrho(f)), B(\varrho(f))),
$$

with $F_{\mu \nu}(z, f, \varrho, b, B) \in \mathbb{C}, C^{\infty}$ in $\zeta \in \mathbb{C}^{m}, f \in \Sigma_{-4 k_{1}}, \varrho \in \mathbb{R}^{4}$ and in $(b, B)$. Furthermore $\left|F_{\mu \nu}\right| \leq C|z|^{M_{0}-1}\left(|z|+\|f\|_{\Sigma_{-4 k_{1}}}\right)$. The contribution in (12.9) is

$$
\left(z^{\mu} \bar{z}^{\nu}+F_{\mu \nu}\right)\left\langle F\left(z^{\prime}, f^{\prime}, \varrho\left(f^{\prime}\right)\right) \mid B_{\mu \nu}(\varrho(f)) f\right\rangle .
$$

Then proceeding as in Lemma 12.2 we get a contribution like on the rhs of (12.8).

Lemma 12.4. Let $\widehat{\mathcal{R}}_{d}=\widehat{\mathcal{R}}_{d}(z, f, \varrho)$ be $C^{k_{0}+2}$ in $z \in \mathbb{C}^{m}, f \in \Sigma_{-4 k_{1}}$ and $\varrho \in \mathbb{R}^{4}$ with values in $\mathbb{R}$ for $d \geq 2$. Suppose that the following inequalities hold:

$$
\begin{aligned}
& \left|\widehat{\mathcal{R}}_{d}(z, f, \varrho)\right| \leq C\|f\|_{\Sigma_{-4 k_{1}}}^{d}, \\
& \left|\widehat{\mathcal{R}}_{2}(z, f, \varrho)\right| \leq C\left(|z|+|\varrho|+\|f\|_{\Sigma_{-4 k_{1}}}\right)\|f\|_{\Sigma_{-4 k_{1}}}^{2} .
\end{aligned}
$$

Then for any $d=2, \ldots, 5$ and for any pair $\left(k_{2}, k_{3}\right)$ there are $k_{1}(d)$ and $k_{0}(d)$ such that for $k_{1} \geq k_{1}(d), k_{0} \geq k_{0}(d)$ and $k_{1} \geq C k_{0}$ for some fixed large constant $C$, then the following occurs: if $d \geq 3, \widehat{\mathcal{R}}_{d}\left(z^{\prime}, f^{\prime}, \varrho\left(f^{\prime}\right)\right)$ is of the form $\mathcal{R}^{\left(M_{0}+1\right)}$; if $d=2$ we have

$$
\widehat{\mathcal{R}}_{2}\left(z^{\prime}, f^{\prime}, \varrho\left(f^{\prime}\right)\right)=\widehat{\mathcal{R}}_{2}(z, f, \varrho(f))+\text { rhs as in (12.4) } \text {. }
$$

Proof. We will only sketch the case $d=2$, the others being similar. Schematically $\widehat{\mathcal{R}}_{2}=G f^{2}$, with $G(z, f, \varrho) \in C^{k_{0}}$ with values in $B^{2}\left(\Sigma_{-4 k_{1}}, \mathbb{C}\right)$ and with $\|G(z, f, \varrho)\|_{B^{2}\left(\Sigma_{-4 k_{1}}, \mathbb{C}\right)}=O\left(|z|+|\varrho|+\|f\|_{\Sigma_{-4 k_{1}}}\right)$. We have

$$
\begin{aligned}
& G\left(z^{\prime}, f^{\prime}, \varrho\left(f^{\prime}\right)\right)\left(f^{\prime}\right)^{2}=G\left(z^{\prime}, f^{\prime}, \varrho\left(f^{\prime}\right)\right) \mathcal{G}^{2} \\
& +2 G\left(z^{\prime}, f^{\prime}, \varrho\left(f^{\prime}\right)\right) \mathcal{G} e^{\mathrm{i} \sigma_{3} \gamma} \tau_{\mathbf{A}} f+G\left(z^{\prime}, f^{\prime}, \varrho\left(f^{\prime}\right)\right)\left(e^{\mathrm{i} \sigma_{3} \gamma} \tau_{\mathbf{A}} f\right)^{2} .
\end{aligned}
$$

The last term can be easily seen to be of the form $\widehat{\mathcal{R}}_{2}^{\left(M_{0}+1\right)}$. The first two terms can be treated as in Lemmas 12.2 and 12.3. For example, for an $\widetilde{\mathbf{R}}$ which can be absorbed in $\mathbf{R}$, we have

$$
G\left(z^{\prime}, f^{\prime}, \varrho\left(f^{\prime}\right)\right) \mathcal{G}^{2}=G(0,0, \varrho(f)) \mathbf{G}_{1}^{2}+\widetilde{\mathbf{R}} .
$$

This follows from the same argument used for (12.6). The first term on the rhs yields a term like the rhs of (12.4) by exactly the same argument used for (12.7), this time using the second inequality in (12.15).

Lemma 12.5. Let

$$
\psi=\sum_{|\mu+\nu|=M+1} d_{\mu \nu}(\varrho(f)) z^{\mu} \bar{z}^{\nu}+\sum_{|\mu+\nu|=M} z^{\mu} \bar{z}^{\nu}\left\langle\sigma_{1} \sigma_{3} D_{\mu \nu}(\varrho(f)) \mid f\right\rangle
$$

be another polynomial like $\chi$, in particular with $d_{\mu \nu} \in C^{k_{0}}\left(\mathbb{R}^{4}, \mathbb{C}\right)$ and $D_{\mu \nu} \in$ $C^{k_{0}}\left(\mathbb{R}^{4}, \Sigma_{4 k_{1}}\left(\mathbb{R}^{3}, \mathbb{C}^{2}\right)\right)$ with $M_{1} \geq 2$. Then, for any pair $\left(k_{2}, k_{3}\right)$ there are $k_{10}$ and 
$k_{00}$ such that for $k_{1} \geq k_{10}, k_{0} \geq k_{00}$ and $k_{1} \geq C k_{0}$ for some fixed large constant $C$, then we have, for an $\boldsymbol{R}$ as in Lemma 12.2.

$$
\psi \circ \phi-\psi=\boldsymbol{R} \text {. }
$$

Proof. We have $\psi \circ \phi=\psi+\int_{0}^{1}\{\psi, \chi\} \circ \phi^{t} d t$. By elementary computation, using (10.6) we get

$$
\begin{aligned}
& \{\psi, \chi\}=\mathrm{i} \partial_{\bar{j}} \psi \partial_{j} \chi-\mathrm{i} \partial_{\bar{j}} \chi \partial_{j} \psi+\mathrm{i} z^{\alpha+\mu} \bar{z}^{\beta+\nu}\left\langle D_{\alpha \beta} \mid \sigma_{1} \sigma_{3} B_{\mu \nu}\right\rangle \\
& +\partial_{\varrho_{i}} \psi z^{\mu} \bar{z}^{\nu}\left\langle\sigma_{1} \sigma_{3} B_{\mu \nu} \mid\left\{\varrho_{i}(f), f\right\}\right\rangle-\partial_{\varrho_{i}} \chi z^{\alpha} \bar{z}^{\beta}\left\langle\sigma_{1} \sigma_{3} D_{\alpha \beta} \mid\left\{\varrho_{i}(f), f\right\}\right\rangle \\
& +\left(\partial_{\varrho_{i}} \psi \partial_{\varrho_{j}} \chi-\partial_{\varrho_{j}} \psi \partial_{\varrho_{i}} \chi\right)\left\{\varrho_{i}(f), \varrho_{j}(f)\right\} .
\end{aligned}
$$

By formula (10.19) all the terms in the last line are of the form $\widehat{\mathcal{R}}_{2}$ as in Lemma 12.4. Then by Lemma 12.4 we have

$$
\widehat{\mathcal{R}}_{2} \circ \phi^{t}=\widehat{\mathcal{R}}_{2}+\text { rhs as in }\left(\text { (12.4) }_{t},\right.
$$

where the last term depends on $t$. Integrating in $t$ we eliminate this dependence. Hence the terms from the last line of (12.19) are absorbed in $\mathbf{R}$. A similar conclusion holds for the terms from the second line of (12.19), this time using the last line of (10.6). Finally, the first line of (12.19) is of the form

$$
h=\sum_{|\mu+\nu|=M+M_{0}} h_{\mu \nu}(\varrho) z^{\mu} \bar{z}^{\nu}+\sum_{|\mu+\nu|=M+M_{0}-1} z^{\mu} \bar{z}^{\nu}\left\langle\sigma_{1} \sigma_{3} H_{\mu \nu}(\varrho) \mid f\right\rangle
$$

with coefficients $h_{\mu \nu} \in C^{k_{0}}\left(\mathbb{R}^{4}, \mathbb{C}\right)$ and $H_{\mu \nu} \in C^{k_{0}}\left(\mathbb{R}^{4}, \Sigma_{4 k_{1}}\left(\mathbb{R}^{3}, \mathbb{C}^{2}\right)\right)$. Then $\int_{0}^{1} h \circ$ $\phi^{t} d t$ can be absorbed in $\mathbf{R}$.

Lemma 12.6. Let $F=F(z, f, \eta, \varrho)$ be $C^{k_{0}}$ in $z \in \mathbb{C}^{m}, f \in \Sigma_{-4 k_{1}}, \eta \in \mathbb{C}^{2}$ and $\varrho \in \mathbb{R}^{4}$ with values in $\Sigma_{4 k_{1}}\left(\mathbb{R}^{3}, B^{d}(\mathbb{C}, \mathbb{C})\right)$. If $d=2$ let $F(0,0,0,0,0)=0$. Set

$$
R(z, f, \varrho)=\int_{\mathbb{R}^{3}} F(x, z, f, f(x), \varrho) f^{d}(x) d x .
$$

Then, for any $d=2, \ldots, 5$ and for any pair $\left(k_{2}, k_{3}\right)$ there are $k_{1}(d)$ and $k_{0}(d)$ such that for $k_{1} \geq k_{1}(d), k_{0} \geq k_{0}(d), k_{1} \geq C k_{0}$ for some fixed large constant $C$ and for $k_{\mu \nu}, K_{\mu \nu}$ and $\boldsymbol{R}$ as in Lemma 12.2, we have

$$
\begin{aligned}
& R \circ \phi=\sum_{|\mu+\nu|=M_{0}+1} k_{\mu \nu}(\varrho(f), b(\varrho(f)), B(\varrho(f))) z^{\mu} \bar{z}^{\nu} \\
& +\sum_{|\mu+\nu|=M_{0}} z^{\mu} \bar{z}^{\nu}\left\langle\sigma_{1} \sigma_{3} K_{\mu \nu}(\varrho(f), b(\varrho(f)), B(\varrho(f))) \mid f\right\rangle+\boldsymbol{R} .
\end{aligned}
$$

If $d>2$ formula (12.21) holds with only $\boldsymbol{R}$ on the rhs.

Proof. The rhs of (12.21) can be written as a sum of terms of the form for $0 \leq i \leq d$

$$
\int_{\mathbb{R}^{3}} F\left(x, z^{\prime}, f^{\prime}, e^{\sigma_{3} \gamma} \tau_{\mathbf{A}} f(x)+\mathcal{G}(x), \varrho\left(f^{\prime}\right)\right) \mathcal{G}^{d-i}(x)\left(e^{\sigma_{3} \gamma} \tau_{\mathbf{A}} f\right)^{i} d x .
$$

The terms with $i \geq 2$ satisfy the statement if we change the variable of integration. In particular for $i=2$ we have $F=0$ at $(z, f)=(0,0)$, where we exploit $\mathcal{G}=0$. 
If $d \geq 3$, then (12.22) can be incorporated in $\mathbf{R}$. We then consider the case $d=2$ and $i=0,1$. For $i=0,(12.22)$ is a sum of the form

$$
\begin{aligned}
& \int_{\mathbb{R}^{3}} F\left(x, z^{\prime}, f^{\prime}, \mathcal{G}(x), \varrho\left(f^{\prime}\right)\right) \mathcal{G}^{2}(x) d x \\
& +\int_{\mathbb{R}^{3}} G\left(x+\mathbf{A}, z^{\prime}, f^{\prime}, e^{\sigma_{3} \gamma} f(x), \tau_{-\mathbf{A}} \mathcal{G}(x), \varrho\left(f^{\prime}\right)\right) \mathcal{G}^{2}(x+\mathbf{A}) e^{\sigma_{3} \gamma} f(x) d x,
\end{aligned}
$$

where the second line can be incorporated in $\mathbf{R}$ and the first line is as in Lemma 12.2. If in (12.22), we have $d=2$ and $i=1$, we have an expression of the form

$$
\begin{aligned}
& \int_{\mathbb{R}^{3}} F\left(x, z^{\prime}, f^{\prime}, \mathcal{G}(x), \varrho\left(f^{\prime}\right)\right) \mathcal{G}(x) e^{\sigma_{3} \gamma} \tau_{\mathbf{A}} f(x) d x \\
& +\int_{\mathbb{R}^{3}} G\left(x+\mathbf{A}, z^{\prime}, f^{\prime}, e^{\sigma_{3} \gamma} f(x), \mathcal{G}(x+\mathbf{A}), \varrho\left(f^{\prime}\right)\right)\left(e^{\sigma_{3} \gamma} f(x)\right)^{2} d x .
\end{aligned}
$$

The second line is absorbed in $\mathbf{R}$. The first line is

$$
\begin{aligned}
& \int_{\mathbb{R}^{3}} F\left(x+\mathbf{A}, z^{\prime}, f^{\prime}, \tau_{-\mathbf{A}} \mathcal{G}(x), \varrho\left(f^{\prime}\right)\right) \mathcal{G}(x+\mathbf{A}) e^{\sigma_{3} \gamma} f(x) d x \\
& =\int_{\mathbb{R}^{3}} F\left(x, z^{\prime}, f^{\prime}, 0, \varrho\left(f^{\prime}\right)\right) \mathcal{G}(x) f(x) d x+\widetilde{\mathbf{R}}
\end{aligned}
$$

where $\widetilde{\mathbf{R}}$ can be absorbed in $\mathbf{R}$. So we can apply Lemma 12.3

12.2. Proof of Theorem 12.1; The step $\ell=2$. At this stage our goal is to obtain a Hamiltonian similar to $H$ but with $\widetilde{\mathcal{R}^{(1)}}=0$ in (9.7). In Lemma 9.2 we can assume $M$ arbitrarily large. We consider a polynomial $\chi$, initially unknown, like in (10.7) with $M_{0}=1$ and with $k_{0}$ and $k_{1}$ arbitrarily large with $1 \ll k_{0} \ll k_{1} \ll M$. We choose $2 N \ll k_{2}(2) \ll k_{3}(2) \ll k_{1}-k_{0}$ with $k_{2}(2)$ as large as needed. We write

$$
H \circ \phi=\left(\psi+H_{2}^{(1)}+\widetilde{\mathcal{R}^{(1)}}+\widetilde{\mathcal{R}^{(2)}}\right) \circ \phi
$$

for $\phi$ the Lie transform of $\chi$. We have

$$
H_{2}^{(1)} \circ \phi=H_{2}^{(1)}+\int_{0}^{1}\left\{H_{2}^{(1)}, \chi\right\} \circ \phi^{t} d t
$$

By the computations in Section 10 we have schematically, for $\ell=1$,

$$
\begin{aligned}
& \left\{H_{2}^{(\ell)}, \chi\right\}=\mathrm{i} \sum_{|\mu+\nu|=\ell+1} \lambda^{(\ell)}(\varrho) \cdot(\mu-\nu) z^{\mu} \bar{z}^{\nu} b_{\mu \nu} \\
& +\mathrm{i} \sum_{|\mu+\nu|=\ell} z^{\mu} \bar{z}^{\nu}\left\langle f \mid \sigma_{1} \sigma_{3}\left(\lambda^{(\ell)}(\varrho) \cdot(\mu-\nu)-\mathcal{H}\right) B_{\mu \nu}\right\rangle \\
& +\sum_{\substack{|\alpha+\beta|=2 \\
(\alpha, \beta) \neq\left(\delta_{j}, \delta_{j}\right) \forall j}} a_{\alpha \beta}^{(\ell)}(\varrho) \sum_{\mu \nu}\left(b_{\mu \nu}+\left\langle\sigma_{1} \sigma_{3} B_{\mu \nu} \mid f\right\rangle\right)\left\{z^{\alpha} \bar{z}^{\beta}, z^{\mu} \bar{z}^{\nu}\right\}+L,
\end{aligned}
$$


with $L$ as in (10.18) with $\chi_{1}$ replaced by $\chi$. Then, in the notation of (10.17) and for $\phi_{0}^{t}$ the flow of the simplified system (11.12), we have

$$
\begin{aligned}
& H_{2}^{(1)} \circ \phi-H_{2}^{(1)} \\
& =\sum_{|\mu+\nu|=2} \lambda^{(1)}(\varrho(f)) \cdot(\mu-\nu) b_{\mu \nu}(\rho(f)) \int_{0}^{1}\left(z^{\mu} \bar{z}^{\nu}\right) \circ \phi_{0}^{t} d t \\
& +\sum_{|\mu+\nu|=1}\left\langle\sigma_{1} \sigma_{3} B_{\mu \nu}(\varrho(f)),(\lambda(\varrho(f)) \cdot(\mu-\nu)+\mathcal{H}) \int_{0}^{1}\left(z^{\mu} \bar{z}^{\nu} f\right) \circ \phi_{0}^{t} d t\right\rangle \\
& +\sum_{\substack{|\alpha+\beta|=2 \\
(\alpha, \beta) \neq\left(\delta_{j}, \delta_{j}\right) \forall j}} a_{\alpha \beta}^{(1)}(\varrho(f))\left[\sum_{|\mu+\nu|=2} b_{\mu \nu}(\varrho(f)) \int_{0}^{1}\left\{z^{\alpha} \bar{z}^{\beta}, z^{\mu} \bar{z}^{\nu}\right\} \circ \phi_{0}^{t} d t\right. \\
& \left.+\sum_{|\mu+\nu|=1}\left\langle\sigma_{1} \sigma_{3} B_{\mu \nu}(\varrho(f)) \mid \int_{0}^{1}\left(f\left\{z^{\alpha} \bar{z}^{\beta}, z^{\mu} \bar{z}^{\nu}\right\}\right) \circ \phi_{0}^{t} d t\right\rangle\right] \\
& +\widehat{\mathbf{R}}_{1}+\int_{0}^{1}\left(\left\langle V_{j}(\varrho(f)) f \mid f\right\rangle \tilde{\chi}_{j}\right) \circ \phi^{t} d t,
\end{aligned}
$$

where by Lemmas 11.3 and 11.4 and by $\widehat{\mathbf{R}}_{1}=O\left(|z|+\|f\|_{\Sigma_{4 k_{0}+4-4 k_{1}}}\right)^{3}$ s.t. $\widehat{\mathbf{R}}_{1}$ is $C^{k_{0}-1}$ in $z \in \mathbb{C}^{m}, f \in \Sigma_{4 k_{0}+4-4 k_{1}}$ and $\varrho(f)$. In particular we have used estimates like

$$
\begin{aligned}
& \left|\left\langle\sigma_{1} \sigma_{3} B_{\mu \nu}(\varrho(f)) \mid \mathcal{H} f \circ \phi^{t}-\mathcal{H} f \circ \phi_{0}^{t}\right\rangle\right| \leq\left\|B_{\mu \nu}(\varrho(f))\right\|_{\Sigma_{4 k_{1}}} \\
& \times\left\|\mathcal{H} f \circ \phi^{t}-\mathcal{H} f \circ \phi_{0}^{t}\right\|_{\Sigma_{-4 k_{1}}} \leq C\left\|f \circ \phi^{t}-f \circ \phi_{0}^{t}\right\|_{\Sigma_{2-4 k_{1}}} \\
& \leq C^{\prime}\left(|z|+\|f\|_{\Sigma_{3-4 k_{1}}}\right)^{2},
\end{aligned}
$$

with the latter a consequence of Lemma 11.4 In (12.25) the last term is as in Lemma 10.3. It can be treated by Lemma 12.4. By our choice of $k_{2}(2)$ and $k_{3}(2)$, if we denote by $\widetilde{\mathbf{R}}_{1}$ the last line of $(12.25)$, we conclude that $\widetilde{\mathbf{R}}_{1}$ can be absorbed in $\mathcal{R}_{0}^{(2)}+\mathcal{R}_{1}^{(2)}+\widehat{\mathcal{R}}_{2}^{(2)}$. We then obtain for $\ell=1$,

$$
\begin{aligned}
& H_{2}^{(\ell)} \circ \phi=H_{2}^{(\ell)}+\mathrm{i} \sum_{|\mu+\nu|=\ell+1} b_{\mu \nu}^{(\ell)}(\varrho(f)) \lambda(\varrho(f)) \cdot(\mu-\nu) z^{\mu} \bar{z}^{\nu} \\
& -\mathrm{i} \sum_{|\mu+\nu|=\ell} z^{\mu} \bar{z}^{\nu}\left\langle f \mid \sigma_{1} \sigma_{3}(\mathcal{H}-\lambda \cdot(\mu-\nu)) B_{\mu \nu}^{(\ell)}(\varrho(f))\right\rangle \\
& +\sum_{|\mu+\nu|=\ell+1} k_{\mu \nu}^{(\ell)}(\varrho(f), b(\varrho(f)), B(\varrho(f))) z^{\mu} \bar{z}^{\nu} \\
& +\sum_{|\mu+\nu|=\ell} z^{\mu} \bar{z}^{\nu}\left\langle\sigma_{1} \sigma_{3} K_{\mu \nu}^{(\ell)}(\varrho(f), b(\varrho(f)), B(\varrho(f))) \mid f\right\rangle+\mathbf{R}_{\ell},
\end{aligned}
$$

where: $\ell=1 ; \mathbf{R}_{1}$ is like $\widetilde{\mathbf{R}}_{1} ; k_{\mu \nu}(0, b, B)=0$ and $K_{\mu \nu}(0, b, B)=0$ (follows by (9.8)); $k_{\mu \nu}(\varrho, b, B) \in \mathbb{C}$ and $K_{\mu \nu}(\varrho, b, B) \in \Sigma_{4 k_{1}}$ are $C^{k_{0}}$ in $\varrho$, in $b_{\mu \nu} \in \mathbb{C}$ and in $B_{\mu \nu} \in \Sigma_{4 k_{1}}$. Notice that to get $C^{k_{0}}$ regularity it is crucial to use $\phi_{0}^{t}$ and its properties stated under (11.13). The $C^{k_{0}}$ regularity is key for the homological equation. 
By Lemma 12.5 we have

$$
\begin{aligned}
& \widetilde{\mathcal{R}^{(1)}} \circ \phi-\widetilde{\mathcal{R}^{(1)}}=\sum_{|\mu+\nu|=2} \widetilde{k}_{\mu \nu}(\varrho(f), b(\varrho(f)), B(\varrho(f))) z^{\mu} \bar{z}^{\nu} \\
& +\sum_{|\mu+\nu|=1} z^{\mu} \bar{z}^{\nu}\left\langle\sigma_{1} \sigma_{3} \widetilde{K}_{\mu \nu}(\varrho(f), b(\varrho(f)), B(\varrho(f))) \mid f\right\rangle+\widetilde{\mathbf{S}}_{1}
\end{aligned}
$$

where: $\widetilde{\mathbf{S}}_{1}$ is like $\mathcal{R}^{(2)}$ and $\widetilde{k}_{\mu \nu}$ (resp. $\widetilde{K}_{\mu \nu}$ ) is like $k_{\mu \nu}$ (resp. $K_{\mu \nu}$ ).

By Lemma 11.5 we have

$$
\psi(\varrho(f)) \circ \phi=\psi(\varrho(f)) \circ \phi_{0}+\mathbf{T}_{\ell}
$$

for $\ell=1$, where $\mathbf{T}_{1}$ is like $\mathcal{R}^{(2)}$. By Lemma 8.7 applied to $\phi_{0}^{t}$, exploiting the fact that $\psi(0)=0$, we have that

$$
\begin{aligned}
& \psi(\varrho(f)) \circ \phi_{0}-\psi(\varrho(f))=\sum_{|\mu+\nu|=\ell+1} k_{\mu \nu}^{(\ell) \prime}(\varrho(f), b(\varrho(f)), B(\varrho(f))) z^{\mu} \bar{z}^{\nu} \\
& +\sum_{a=1}^{3} \sum_{i=0}^{1} \sum_{|\mu+\nu|=\ell} z^{\mu} \bar{z}^{\nu}\left\langle\sigma_{1} \sigma_{3} \partial_{a}^{i} K_{\mu \nu}^{(\ell) i a}(\varrho(f), b(\varrho(f)), B(\varrho(f))) \mid f\right\rangle+\mathbf{R}_{\ell}^{\prime},
\end{aligned}
$$

with $\mathbf{R}_{\ell}^{\prime}$ like $\mathbf{R}_{\ell}$.

By Lemma 12.6

$$
\begin{aligned}
& \left\langle B_{2}\left(z^{\prime}, \varrho\left(f^{\prime}\right)\right) \mid\left(f^{\prime}\right)^{2}\right\rangle=\widehat{\mathbf{S}}_{1} \\
& +\sum_{|\mu+\nu|=2} v_{\mu \nu}(\varrho(f), b(\varrho(f)), B(\varrho(f))) z^{\mu} \bar{z}^{\nu} \\
& +\sum_{|\mu+\nu|=1} z^{\mu} \bar{z}^{\nu}\left\langle\sigma_{1} \sigma_{3} \Upsilon_{\mu \nu}(\varrho(f), b(\varrho(f)), B(\varrho(f))) \mid f\right\rangle,
\end{aligned}
$$

where $v_{\mu \nu}$ and $\Upsilon_{\mu \nu}$ have the same properties as $k_{\mu \nu}, K_{\mu \nu}$ and $\widehat{\mathbf{S}}_{1}$ is like $\mathcal{R}^{(2)}$. Notice that the fact that $v_{\mu \nu}$ and $\Upsilon_{\mu \nu}$ are $C^{k_{0}}$ is key here for the homological equation.

By Lemma 12.4, where we are using $k_{0} \ll k_{1} \ll M$, which is much more than needed, we have for $\ell=1$,

$$
\begin{gathered}
\widehat{\mathcal{R}}_{2}^{(\ell)} \circ \phi=\widehat{\mathcal{R}}_{2}^{(\ell)}+\sum_{|\mu+\nu|=\ell+1} \kappa_{\mu \nu}^{(\ell)}(\varrho(f), b(\varrho(f)), B(\varrho(f))) z^{\mu} \bar{z}^{\nu} \\
+\sum_{|\mu+\nu|=\ell} z^{\mu} \bar{z}^{\nu}\left\langle\sigma_{1} \sigma_{3} \mathcal{K}_{\mu \nu}^{(\ell)}(\varrho(f), b(\varrho(f)), B(\varrho(f))) \mid f\right\rangle+\widehat{\mathbf{S}}_{\ell},
\end{gathered}
$$

where $\kappa_{\mu \nu}^{(1)}, \mathcal{K}_{\mu \nu}^{(1)}$ and $\widehat{\mathbf{S}}_{1}^{(1)}$ are like $k_{\mu \nu}, K_{\mu \nu}$ and $\mathcal{R}^{(2)}$. Now consider $\mathbf{K}:=\widetilde{\mathcal{R}^{(1)}}$ and $\widetilde{\mathbf{K}}$ the polynomial of the form (10.9) with

$$
\begin{aligned}
\widetilde{\mathbf{k}}_{\mu \nu}(\varrho, b, B) & :=\left(k_{\mu \nu}+k_{\mu \nu}^{(1) \prime}+\widetilde{k}_{\mu \nu}+v_{\mu \nu}+\kappa_{\mu \nu}^{(1)}\right)(\varrho, b, B), \\
\widetilde{\mathbf{K}}_{\mu \nu}(\varrho, b, B) & :=\left(K_{\mu \nu}+\widetilde{K}_{\mu \nu}+\sum_{i=0}^{1} \sum_{a=1}^{3} \partial_{a}^{i} K_{\mu \nu}^{(1) i a}+\Upsilon_{\mu \nu}+\mathcal{K}_{\mu \nu}^{(1)}\right)(\varrho, b, B) .
\end{aligned}
$$

Then $\mathbf{K}(\varrho)$ and $\widetilde{\mathbf{K}}(\varrho, b, B)$ are as in Lemmas 10.2,10.3. This means that we can choose $\chi$ in (11.1) so that

$$
\begin{aligned}
& \mathrm{i} \lambda(\varrho) \cdot(\mu-\nu) z^{\mu} \bar{z}^{\nu} b_{\mu \nu}+z^{\mu} \bar{z}^{\nu} \mathrm{i}\left\langle f \mid \sigma_{1} \sigma_{3}(\lambda(\varrho), \cdot(\mu-\nu)-\mathcal{H}) B_{\mu \nu}\right\rangle \\
& +\widetilde{\mathcal{R}^{(1)}}+\widetilde{\mathbf{K}}(\varrho(f), b(\varrho(f)), B(\varrho(f)))=Z_{1}(\varrho(f)),
\end{aligned}
$$


where $Z_{1}(\varrho)$ is in normal form and homogeneous of degree 2 in $(z, \bar{z}, f)$ and with $Z_{1}(0)=0$.

For $d>2$, the terms $\left\langle B_{d}\left(z^{\prime}, \varrho\left(f^{\prime}\right)\right) \mid\left(f^{\prime}\right)^{d}\right\rangle$ can be incorporated in $\mathcal{R}^{(2)}$ by Lemma 12.6. This is also true for the $d=5$ term and for $E_{P}\left(f^{\prime}\right)$. We set

$$
\begin{aligned}
\widetilde{\mathcal{R}}^{(2)}: & =\widehat{\mathcal{R}}_{2}^{(1)}+\mathbf{R}_{1}+\widehat{\mathbf{S}}_{1}+\widetilde{\mathbf{S}}_{1}+\mathbf{T}_{1}+\mathbf{R}_{1}^{\prime} \\
& +\widetilde{\mathcal{R}^{(2)}} \circ \phi-\left\langle B_{2}\left(z^{\prime}, \varrho\left(f^{\prime}\right)\right) \mid\left(f^{\prime}\right)^{2}\right\rangle-\widehat{\mathcal{R}}_{2}^{(1)} \circ \phi .
\end{aligned}
$$

All the terms on the rhs have the properties required for $\mathcal{R}^{(2)}$. Hence, if we also set $\mathcal{R}^{(2)}:=\widetilde{\mathcal{R}}^{(2)}$, we conclude the proof of case $\ell=2$ in Theorem 12.1.

12.3. Proof of Theorem 12.1: The step $\ell>2$. Case $\ell=2$ has been treated in Subsection 12.2. We proceed by induction to complete the proof of Theorem 12.1. From the argument below one can see that $H_{2}^{(\ell)}=H_{2}^{(2)}$ for all $\ell \geq 2$. Suppose that the statement of Theorem 12.1 holds for an $\ell \geq 2$.

Set $k_{0}=k_{2}(\ell)-\underline{k}$ and $k_{1}=k_{3}(\ell)-\underline{k}$ for a fixed and appropriately large $\underline{k}$. We will choose $2 N \ll k_{2}(\ell+1) \ll k_{3}(\ell+1) \ll k_{1}-k_{0}$ with $k_{2}(\ell+1)$ as large as needed.

Since $H^{(\ell)}=H \circ \mathcal{T}_{\ell}$ is real valued (because $H$ is real valued), $a_{\mu \nu}^{(\ell)}$ and $G_{\mu \nu}^{(\ell)}$ satisfy (4.12). We seek an appropriate polynomial $\chi$ as in (11.1) with $M_{0}=\ell$. For any such polynomial, we consider its Lie transform $\phi=\phi^{1}$. Proceeding as in the previous step of the proof, we obtain formula (12.27) with: $\mathbf{R}_{\ell}=O\left(|z|+\|f\|_{\Sigma_{-4 k_{1}+2}}\right)^{\ell+2}$, with $\mathbf{R}_{\ell} \in C^{k_{0}-1}$ in $z \in \mathbb{C}^{m}, f \in \Sigma_{4 k_{0}-4 k_{1}}$ and $\rho(f) ; k_{\mu \nu}^{(\ell)}(0, b, B)=0$, resp. $K_{\mu \nu}^{(\ell)}(0, b, B)=0 ; \widehat{k}_{\mu \nu}^{(\ell)}(\varrho, b, B) \in \mathbb{C}$ and $\widehat{K}_{\mu \nu}^{(\ell)}(\varrho, b, B) \in \Sigma_{4 k_{1}}$ are $C^{k_{0}}$ in $\varrho \in \mathbb{R}^{4}$, $b_{\mu \nu} \in \mathbb{C}$ and $B_{\mu \nu} \in \Sigma_{4 k_{1}}$. By our choice of $k_{2}(\ell+1)$ and $k_{3}(\ell+1), \mathbf{R}_{\ell}$ can be absorbed in $\mathcal{R}_{0}^{(\ell+1)}+\mathcal{R}_{1}^{(\ell+1)}+\widehat{\mathcal{R}}_{2}^{(\ell+1)}$.

By Lemma 12.6 we have

$$
\begin{aligned}
& \left\langle F_{2}^{(\ell)}\left(z^{\prime}, f^{\prime}, f^{\prime}(\cdot), \varrho\left(f^{\prime}\right)\right) \mid\left(f^{\prime}\right)^{2}\right\rangle=\left\langle F_{2}^{(\ell)}(z, f, f(\cdot), \varrho(f)) \mid f^{2}\right\rangle \\
& +\widehat{\mathbf{S}}_{\ell}+\sum_{|\mu+\nu|=\ell+1} v_{\mu \nu}^{(\ell)}(\varrho(f), b(\varrho(f)), B(\varrho(f))) z^{\mu} \bar{z}^{\nu} \\
& +\sum_{|\mu+\nu|=\ell} z^{\mu} \bar{z}^{\nu}\left\langle\sigma_{1} \sigma_{3} \Upsilon_{\mu \nu}^{(\ell)}(\varrho(f), b(\varrho(f)), B(\varrho(f))) \mid f\right\rangle,
\end{aligned}
$$

where $v_{\mu \nu}^{(\ell)}\left(\right.$ resp. $\left.\Upsilon_{\mu \nu}^{(\ell)}\right)$ is like $k_{\mu \nu}^{(\ell)}$ (resp. $\left.K_{\mu \nu}^{(\ell)}\right)$ and $\widehat{\mathbf{S}}_{\ell}$ is das like $\mathcal{R}^{(\ell+1)}$. Proceeding as in (12.32), by Lemma 12.4 we get

$$
\begin{aligned}
& \widehat{\mathcal{R}}_{2}^{(\ell)} \circ \phi-\widehat{\mathcal{R}}_{2}^{(\ell)}=\sum_{|\mu+\nu|=\ell+1} \kappa_{\mu \nu}^{(\ell)}(\varrho(f), b(\varrho(f)), B(\varrho(f))) z^{\mu} \bar{z}^{\nu} \\
& +\sum_{|\mu+\nu|=\ell} z^{\mu} \bar{z}^{\nu}\left\langle\sigma_{1} \sigma_{3} \mathcal{K}_{\mu \nu}^{(\ell)}(\varrho(f), b(\varrho(f)), B(\varrho(f))) \mid f\right\rangle+\widehat{\mathbf{S}}_{\ell}
\end{aligned}
$$

with $\widetilde{\mathbf{S}}_{\ell}$ like $\mathcal{R}^{(\ell+1)}, \widetilde{k}_{\mu \nu}^{(\ell)}$, resp. $\widetilde{K}_{\mu \nu}^{(\ell)}$, with the properties of $k_{\mu \nu}^{(\ell)}$, resp. $K_{\mu \nu}^{(\ell)}$. Proceeding as for the $\ell=2$ case, we have that $\psi(\varrho(f)) \circ \phi-\psi(\varrho(f))$ is like the rhs of (12.29). 
Set $\mathbf{K}^{(\ell)}(\varrho(f)):=\mathcal{R}_{0}^{(\ell)}+\mathcal{R}_{1}^{(\ell)}$. Consider the polynomial $\widetilde{\mathbf{K}}$ of the form (10.9) with coefficients

$$
\begin{aligned}
\widetilde{\mathbf{k}}_{\mu \nu}^{(\ell)}(\varrho, b, B) & :=\left(k_{\mu \nu}^{(\ell)}+k_{\mu \nu}^{(\ell) \prime}+\widetilde{k}_{\mu \nu}^{(\ell)}+v_{\mu \nu}^{(\ell)}\right)(\varrho, b, B), \\
\widetilde{\mathbf{K}}_{\mu \nu}^{(\ell)}(\varrho, b, B) & :=\left(K_{\mu \nu}^{(\ell)}+\sum_{i=0}^{1} \sum_{a=1}^{3} \partial_{a}^{i} K_{\mu \nu}^{(\ell) i a}+\mathcal{K}_{\mu \nu}^{(\ell)}+\Upsilon_{\mu \nu}^{(\ell)}\right)(\varrho, b, B) .
\end{aligned}
$$

Then $\mathbf{K}^{(\ell)}(\varrho)$ and $\widetilde{\mathbf{K}}^{(\ell)}(\varrho, b, B)$ are as in Lemma 10.2. This means that we can choose $\chi$ in (11.1) so that

$$
\begin{aligned}
& \mathrm{i} \lambda(\varrho) \cdot(\mu-\nu) z^{\mu} \bar{z}^{\nu} b_{\mu \nu}+z^{\mu} \bar{z}^{\nu} \mathrm{i}\left\langle f \mid \sigma_{1} \sigma_{3}(\lambda(\varrho), \cdot(\mu-\nu)-\mathcal{H}) B_{\mu \nu}\right\rangle \\
& +\mathbf{K}^{(\ell)}(\varrho)+\widetilde{\mathbf{K}}^{(\ell)}(\varrho, b, B)=Z_{\ell}(\varrho),
\end{aligned}
$$

where $Z_{\ell+1}(\varrho)$ is in normal form and homogeneous of degree $\ell+1$ in $(z, \bar{z}, f)$. Set $Z^{(\ell+1)}:=Z^{(\ell)}+Z_{\ell+1}$ and

$$
H^{(\ell+1)}:=H^{(\ell)} \circ \phi=H_{2}^{(\ell)}+Z^{(\ell+1)}+\widetilde{\mathcal{R}}^{(\ell+1)},
$$

where

$$
\begin{aligned}
\widetilde{\mathcal{R}}^{(\ell+1)}: & =Z^{(\ell+1)} \circ \phi-Z^{(\ell+1)} \\
& +\sum_{d=3}^{6} \mathcal{R}_{d}^{(\ell)} \circ \phi+\widetilde{\mathcal{R}}_{2}^{(\ell)}+\mathbf{R}_{\ell}+\widetilde{\mathbf{S}}_{\ell}+\widehat{\mathbf{S}}_{\ell}+\mathbf{R}_{\ell}^{\prime} .
\end{aligned}
$$

The terms in the last line are of the form requested for terms of $\mathcal{R}^{(\ell+1)}$. By Lemma $12.5 Z^{(\ell+1)} \circ \phi-Z^{(\ell+1)}$ is also of the same type. Then we set $\mathcal{R}^{(\ell+1)}:=\widetilde{\mathcal{R}}^{(\ell+1)}$ and the proof is finished.

\section{DiSPERSION}

We apply Theorem 12.1 for $\ell=2 N+1$ (recall $N=N_{1}$, where $N_{j} \lambda_{j}<\omega_{0}<$ $\left.\left(N_{j}+1\right) \lambda_{j}\right)$. In the rest of the paper we work with the Hamiltonian $H^{(2 N+1)}$. We will drop the upper index. So we will set $H=H^{(2 N+1)}, H_{2}=H_{2}^{(2 N+1)}, \lambda_{j}=\lambda_{j}^{(2 N+1)}$, $\lambda=\lambda^{(2 N+1)}, Z_{i}=Z_{i}^{(2 N+1)}$ for $i=0,1$ and $\mathcal{R}=\mathcal{R}^{(2 N+1)}$. In particular we will denote by $G_{\mu \nu}$ the coefficients $G_{\mu \nu}^{(2 N+1)}$ of $Z_{1}^{(2 N+1)}$. We will show:

Theorem 13.1. Consider the constant $0<\epsilon<\varepsilon$ of Theorem 9.1. There is a fixed $C>0$ such that for $\varepsilon$ sufficiently small and for any $\epsilon \in(0, \varepsilon)$ we have

$$
\begin{gathered}
\|f\|_{L_{t}^{p}\left([0, \infty), W_{x}^{1, q}\right)} \leq C \epsilon \text { for all admissible pairs }(p, q), \\
\left\|z^{\mu}\right\|_{L_{t}^{2}([0, \infty))} \leq C \epsilon \text { for all multiindexes } \mu \text { with } \lambda \cdot \mu>\omega_{0}, \\
\left\|z_{j}\right\|_{W_{t}^{1, \infty}([0, \infty))} \leq C \epsilon \text { for all } j \in\{1, \ldots, m\} .
\end{gathered}
$$

Notice that by the time reversibility of the NLS, the above estimates imply the ones with $\mathbb{R}$ replacing $[0, \infty)$, doubling the constants in (13.1)-(13.2).

(13.3) is a consequence of the already known orbital stability, so we do not need to prove it. To obtain Theorem 13.1 it is enough to show that there are fixed constants $C_{1}, C_{2}$ (large) and $\varepsilon$ (small) such that if for $\epsilon \in(0, \varepsilon)$ (where $\epsilon$ and $\varepsilon$ are those of Theorem 9.1)

$$
\begin{gathered}
\|f\|_{L_{t}^{p}\left([0, T], W_{x}^{1, q}\right)} \leq C_{1} \epsilon \text { for all admissible pairs }(p, q), \\
\left\|z^{\mu}\right\|_{L_{t}^{2}([0, T])} \leq C_{2} \epsilon \text { for all multiindexes } \mu \text { with } \omega \cdot \mu>\omega_{0},
\end{gathered}
$$


then in fact (13.4) and (13.5) hold but with $C_{1}, C_{2}$ replaced by $C_{1} / 2, C_{2} / 2$. In fact we conclude that these estimates hold for all $T$, and so (13.1)-(13.2) hold. The proof consists of three main steps.

(i) Estimate $f$ in terms of $z$.

(ii) Substitute the variable $f$ with a new "smaller" variable $g$ and find smoothing estimates for $g$.

(iii) Reduce the system for $z$ to a closed system involving only the $z$ variables, by insulating the part of $f$ which interacts with $z$, and by decoupling the rest (this remainder is $g$ ). Then clarify the nonlinear Fermi golden rule.

13.1. Proof of Theorem 13.1; Step (i). Step (i) is encapsulated by the following proposition:

Proposition 13.2. Assume (13.4)-(13.5). Then there exist constants $C=C\left(C_{1}\right.$, $C_{2}$ ) and $K_{1}=K_{1}\left(C_{1}\right)$, such that, if $C\left(C_{1}, C_{2}\right) \epsilon$ is sufficiently small, then we have

$$
\|f\|_{L_{t}^{p}\left([0, T], W_{x}^{1, q}\right)} \leq K_{1} \epsilon \text { for all admissible pairs }(p, q) \text {. }
$$

Proof. Consider $Z_{1}$ of the form (10.4). Set

$$
G_{\mu \nu}^{0}=G_{\mu \nu}(\varrho(0)), \quad \lambda_{j}^{0}=\lambda_{j}\left(\omega_{0}\right)
$$

Then we have (with finite sums)

$$
\begin{aligned}
& \mathrm{i} \dot{f}-\mathcal{H} f-\left(\partial_{Q(f)} H\right) P_{c}\left(\omega_{0}\right) \sigma_{3} f-\mathrm{i}\left(\partial_{\Pi_{a}(f)} H\right) P_{c}\left(\omega_{0}\right) \partial_{x_{a}} f \\
& =\sum_{\substack{\left|\lambda^{0} \cdot(\nu-\mu)\right|>\omega_{0} \\
|\mu+\nu| \leq 2 N_{1}+1}} z^{\mu} \bar{z}^{\nu} G_{\mu \nu}^{0} \\
& +\sum_{\substack{\left|\lambda^{0} \cdot(\nu-\mu)\right|>m-\omega_{0},|\mu+\nu| \leq 2 N_{1}+1}} z^{\mu} \bar{z}^{\nu}\left(G_{\mu \nu}-G_{\mu \nu}^{0}\right)+\sigma_{3} \sigma_{1} \widehat{\nabla}_{f} \mathcal{R},
\end{aligned}
$$

with $\widehat{\nabla}_{f} \mathcal{R}(z, f, \rho)$ the gradient in $f$, with no differentiation in $\varrho(f)$. In order to obtain bounds on $f$, we need bounds on the right hand term of the equation, especially the last two terms. They are provided by the following lemma.

Lemma 13.3. Assume (13.4)-(13.5). Then there is a constant $C\left(C_{1}, C_{2}\right)$ independent of $\epsilon$ such that the following is true: we have $\sigma_{3} \sigma_{1} \widehat{\nabla}_{f} \mathcal{R}=R_{1}+R_{2}$ with

$$
\left\|R_{1}\right\|_{L_{t}^{1}\left([0, T], H_{x}^{1}\right)}+\left\|R_{2}\right\|_{L_{t}^{2}\left([0, T], W_{x}^{1, \frac{6}{5}}\right)} \leq C\left(C_{1}, C_{2}\right) \epsilon^{2} .
$$

Proof. The proof is standard, a combination of $\mathrm{BC}]$ and $\mathrm{CM}$.

Lemma 13.4. Consider $\mathrm{i} \dot{\psi}-\mathcal{H} \psi-\varphi(t) \sigma_{3} P_{c} \psi-\mathrm{i} A_{a}(t) P_{c} \partial_{x_{a}} \psi=F$, where $P_{c}=$ $P_{c}\left(\omega_{0}\right), \psi=P_{c} \psi, \varphi$ and each $A_{a}$ are real valued. Then there exist $c_{0}>0$ and $C>0$ such that if $\|(\varphi, A)\|_{L_{t}^{\infty}[0, T]}<c_{0}$, then for $(p, q)$ as in Theorem 13.1 we have

$$
\|\psi\|_{L_{t}^{p}\left([0, T], W^{1, q}\right)} \leq C\|\psi(0)\|_{H^{1}}+C\|F\|_{L_{t}^{1}\left([0, T], H_{x}^{1}\right)+L_{t}^{2}\left([0, T], W_{x}^{1, \frac{6}{5}}\right)} \cdot
$$

Proof. This result is due to Beceanu; see for example Theorem 3.8 of [Be]. 
Continuation of the proof of Proposition 13.2. By (13.8) we can apply to $f$ Lemma 13.4 by taking $\varphi(t)=\partial_{Q(f)} H, A_{a}(t)=\partial_{\Pi_{a}(f)} H$ and $F=\operatorname{rhs}(13.8)-\varphi\left[\sigma_{3}, P_{d}\right] f$. Then, for fixed constants,

$$
\begin{aligned}
& \|f\|_{L_{t}^{p}\left([0, T], W^{1, q}\right)} \leq C_{1}\|f(0)\|_{H^{1}}+C_{1}\|F\|_{L_{t}^{1}\left([0, T], H_{x}^{1}\right)+L_{t}^{2}\left([0, T], W_{x}^{1, \frac{6}{5}}\right)} \\
& \leq C_{1}\|f(0)\|_{H^{1}}+C \sum_{\lambda \cdot \mu>m-\omega_{0}}\left\|z^{\mu}\right\|_{L_{t}^{2}(0, T)}^{2} \\
& +C\left\|R_{1}\right\|_{L_{t}^{1}\left([0, T], H_{x}^{1}\right)}+\left\|R_{2}\right\|_{L_{t}^{2}\left([0, T], W_{x}^{1, \frac{6}{5}}\right)}+C \epsilon\|f\|_{L_{t}^{2}\left([0, T], L_{x}^{6}\right)} .
\end{aligned}
$$

For $\epsilon$ small this yields Proposition 13.2 by Lemma 13.4 and by (13.5).

Lemma 13.5. Assume the conclusions of Theorem 13.1. Then there exists a fixed $C>0$ and $f_{+} \in H^{1}$ with $\left\|f_{+}\right\|_{H^{1}}<C \epsilon$ such that we have

$$
\lim _{t \rightarrow+\infty}\left\|\tau_{X(t)} e^{\mathrm{i} \chi(t) \sigma_{3}} f(t)-e^{\mathrm{i} t \Delta \sigma_{3}} f_{+}\right\|_{H^{1}}=0
$$

for $\chi(t):=t \omega_{0}+\int_{0}^{t} \partial_{Q(f)} H\left(t^{\prime}\right) d t^{\prime}$ and $X(t):=\int_{0}^{t} \partial_{\Pi(f)} H\left(t^{\prime}\right) d t^{\prime}$.

Proof. For $\psi(t)=f(t), F=\operatorname{rhs}(13.8)-\varphi(t)\left[\sigma_{3}, P_{d}\right] f, \varphi(t)=\partial_{Q(f)} H, \mathcal{A}_{a}(t)=$ $\partial_{\Pi_{a}(f)} H, \mathcal{U}(t)=e^{\int_{0}^{t}\left(\mathrm{i} \sigma_{3} \varphi(\tau)+\mathcal{A}(\tau) \cdot \nabla\right) d \tau}$ and for $t_{1}<t_{2}$, we have

$$
\begin{aligned}
& \left\|e^{\mathrm{i} \mathcal{H}_{0} t_{2}} \mathcal{U}\left(t_{2}\right) f\left(t_{2}\right)-e^{\mathrm{i} \mathcal{H}_{0} t_{1}} \mathcal{U}\left(t_{1}\right) f\left(t_{1}\right)\right\|_{H^{1}} \\
& \leq\left\|\int_{t_{1}}^{t_{2}} e^{\mathrm{i} \mathcal{H}_{0} t^{\prime}} \mathcal{U}\left(t^{\prime}\right)\left[F\left(t^{\prime}\right)+V f\left(t^{\prime}\right)-\varphi\left(t^{\prime}\right) \sigma_{3} P_{d} f\left(t^{\prime}\right)-\mathrm{i} \mathcal{A}_{a} P_{d} \partial_{a} f\left(t^{\prime}\right)\right] d t^{\prime}\right\|_{H^{1}} \\
& \leq C\left(\sum_{\left|\lambda^{0} \cdot \mu\right|>m-\omega_{0}}\left\|z^{\mu}\right\|_{L^{2}\left(t_{1}, t_{2}\right)}+\left\|R_{1}\right\|_{L_{t}^{1}\left(\left[t_{1}, t_{2}\right], H_{x}^{1}\right)}\right. \\
& \left.+\left\|R_{2}\right\|_{L_{t}^{2}\left(\left[t_{1}, t_{2}\right], W_{x}^{1, \frac{6}{5}}\right)}+\|f\|_{L_{t}^{2}\left(\left[t_{1}, t_{2}\right], W_{x}^{1,6}\right)}\right) .
\end{aligned}
$$

Since the rhs has limit 0 as $t_{1} \rightarrow+\infty$, there exists $f_{+} \in H^{1}$ such that

$$
\lim _{t \rightarrow+\infty}\left\|\mathcal{U}(t) f(t)-e^{-\mathrm{i} \mathcal{H}_{0} t} f_{+}\right\|_{H^{1}}=0
$$

This yields Lemma 13.5.

Lemma 13.6. Assume the conclusions of Theorem 13.1 and the notation of Theorem 9.1. Then the conclusions of Theorem 9.1 hold with the $f_{+}$of (13.12) and with

$$
\widehat{\vartheta}=\chi+\mathrm{i} \sum_{\ell=1}^{2 N+1} \gamma_{\ell}, \quad \widehat{D}=X-\sum_{\ell=1}^{2 N+1} \mathbf{A}_{\ell},
$$

with $\mathbf{A}_{\ell}$ and $\gamma_{\ell}$ the terms in (11.6) corresponding to the Lie transforms $\phi_{\ell}$ of Theorem 12.1 .

Proof. This follows immediately from (11.6). Indeed, schematically

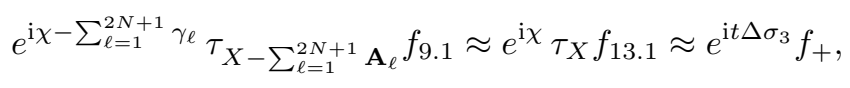

where $\sqrt{9.1}$ (resp. $\sqrt{13.1})$ is the coordinate in Theorem 9.1 (resp. Theorem 13.1). 
Lemma 13.7. Denote by $\left(\omega, v, z^{\prime}, f^{\prime}\right)$ the coordinates (2.13) of the solution in the initial system of coordinates (we omit $\vartheta, D$ ). Then $\lim _{t \rightarrow \infty} z^{\prime}(t)=0$, and there are functions $\theta \in C^{1}(\mathbb{R}, \mathbb{R})$ and $y \in C^{1}\left(\mathbb{R}, \mathbb{R}^{3}\right)$ s.t.

$$
\lim _{t \rightarrow+\infty}\left\|\tau_{y(t)} e^{\mathrm{i} \sigma_{3}\left(v(t) \cdot \frac{x}{2}+\theta(t)\right)} f^{\prime}(t)-e^{\mathrm{i} t \Delta \sigma_{3}} f_{+}\right\|_{H^{1}}=0,
$$

with the $f_{+}$of Lemma 13.5, and $\lim _{t} \nearrow_{\infty} \omega(t)=\omega_{+}$and $\lim _{t} \nearrow_{\infty} v(t)=v_{+}$.

Proof. If we denote by $\left(z^{\prime}, f^{\prime}\right)$ the initial coordinates and by $(49.1, f(9.1)$ the coordinates in (9.2) considered in Theorem 9.1, we have $z^{\prime}=z+O\left(\left.\left|\frac{29.1}{1}+\| \int_{9.1}\right|\right|_{L_{x}^{2,-2}}\right)$. So the asymptotic behavior of $z^{\prime}$ and of 79.1 are the same. By (8.15) we get

$$
\tau_{\widehat{D}} e^{\mathrm{i} \widehat{\vartheta} \sigma_{3}} \sqrt{9.1}=\tau_{\widehat{D}-\mathbf{A}} e^{\sigma_{3}\left(\frac{\mathrm{i}}{2} v \cdot x-\widetilde{\gamma}+\mathrm{i} \widehat{\vartheta}\right)} f^{\prime}-\tau_{\widehat{D}-\mathbf{A}} e^{\sigma_{3}\left(\frac{\mathrm{i}}{2} v \cdot x-\widetilde{\gamma}+\mathrm{i} \widehat{\vartheta}\right)} \mathcal{G} .
$$

By (8.11) the second term on the rhs converges to 0 in $H^{1}$ as $t \nearrow \infty$. Hence, for $\theta:=\widehat{\vartheta}+\mathrm{i} \widetilde{\gamma}$ and for $y:=\widehat{D}-\mathbf{A}$, we obtain (13.14).

We have $q(\omega(t))=q\left(\omega_{0}\right)-\frac{\left\|f^{\prime}(t)\right\|_{2}^{2}}{2}+O\left(\left|z^{\prime}(t)\right|+\left\|f^{\prime}(t)\right\|_{L_{x}^{2,-2}}\right)$ by $q\left(\omega_{0}\right)=q(\omega)+$ $Q(R)$. Then (13.14) and $\left|z^{\prime}(t)\right|+\left\|f^{\prime}(t)\right\|_{L_{x}^{2,-2}} \rightarrow 0$ imply

$$
\lim _{t \rightarrow+\infty} q(\omega(t))=q\left(\omega_{0}\right)-\lim _{t \rightarrow+\infty} \frac{\left\|e^{\mathrm{i} t \sigma_{3} \Delta} f_{+}\right\|_{2}^{2}}{2}=q\left(\omega_{0}\right)-\frac{\left\|f_{+}\right\|_{2}^{2}}{2}=q\left(\omega_{+}\right),
$$

where $\omega_{+}$is the unique element near $\omega_{0}$ for which the last equality holds. So $\lim _{t \rightarrow+\infty} \omega(t)=\omega_{+}$. By $v=2\left(\Pi\left(U_{0}\right)-\Pi(R)\right) Q^{-1}\left(U_{0}\right)$ we obtain

$$
v=2\left(\Pi\left(U_{0}\right)-\Pi\left(f^{\prime}\right)\right) Q^{-1}\left(U_{0}\right)+O\left(\left|z^{\prime}(t)\right|+\left\|f^{\prime}(t)\right\|_{L_{x}^{2,-2}}\right),
$$

which implies $\lim _{t \nearrow_{\infty}} v(t)=2\left(\Pi\left(U_{0}\right)-\Pi\left(f_{+}\right)\right) Q^{-1}\left(U_{0}\right)=: v_{+}$.

Lemma 13.8. For $(\theta, y)$ the functions of (13.14) and $(\vartheta, D)$ the coordinates of (2.11), there are $\vartheta_{0} \in \mathbb{R}, y_{0} \in \mathbb{R}^{3}$ and $o(1) \rightarrow 0$ as $t \rightarrow+\infty$ s.t.

$$
\theta(t)=\vartheta(t)+\vartheta_{0}+o(1), \quad y(t)=D(t)+y_{0} .
$$

Proof. Consider the representation $U=\tau_{D} e^{\mathrm{i} \sigma_{3}\left(\frac{v \cdot x}{2}+\vartheta\right)}\left(\Phi_{\omega}+R\right)$ of the solution of $\mathrm{i} \dot{U}=\sigma_{3} \sigma_{1} \nabla E(U)$. We have the identity

$$
\begin{aligned}
& \mathrm{i} \dot{U}=-\sigma_{3}\left(\dot{\vartheta}-\frac{v \cdot \dot{D}}{2}\right) \tau_{D} e^{\mathrm{i} \sigma_{3} \Theta}\left(\Phi_{\omega}+R\right)-\mathrm{i} \dot{D} \cdot \tau_{D} e^{\mathrm{i} \sigma_{3} \Theta} \nabla_{x}\left(\Phi_{\omega}+R\right) \\
& -\frac{\dot{v}}{2} \cdot \tau_{D} e^{\mathrm{i} \sigma_{3} \Theta} \sigma_{3} x\left(\Phi_{\omega}+R\right)+\mathrm{i} \dot{\omega} \tau_{D} e^{\mathrm{i} \sigma_{3} \Theta} \partial_{\omega} \Phi_{\omega}+\mathrm{i} \tau_{D} e^{\mathrm{i} \sigma_{3} \Theta} \dot{R}
\end{aligned}
$$

By Lemma 4.2 we have

$$
\nabla E(U)=\tau_{D} e^{-\mathrm{i} \sigma_{3}\left(\frac{v \cdot x}{2}+\vartheta\right)}\left(\nabla E\left(\Phi_{\omega}+R\right)-v_{a} \nabla \Pi_{a}\left(\Phi_{\omega}+R\right)+\frac{v^{2}}{4} \nabla Q\left(\Phi_{\omega}+R\right)\right) .
$$

Then, using (2.4) as well, i $\dot{\mathrm{U}}=\sigma_{3} \sigma_{1} \nabla E(U)$ can be expanded for $\varpi=\omega_{0}$ into

$$
\begin{aligned}
& -\sigma_{3}\left(\dot{\vartheta}-\frac{v \cdot \dot{D}}{2}+\frac{v^{2}}{4}-\varpi\right)\left(\Phi_{\omega}+R\right)-\mathrm{i}(\dot{D}-v) \cdot \nabla_{x}\left(\Phi_{\omega}+R\right)+\mathrm{i} \dot{R} \\
& -\frac{\dot{v} \cdot x}{2} \sigma_{3}\left(\Phi_{\omega}+R\right)+\mathrm{i} \dot{\omega} \partial_{\omega} \Phi_{\omega}=\sigma_{3} \sigma_{1}\left(\nabla E\left(\Phi_{\omega}+R\right)+\varpi Q\left(\Phi_{\omega}+R\right)\right) .
\end{aligned}
$$


Using the first system of coordinates (2.14), and denoting the $f$-coordinate by $f^{\prime}$, we have for $\left\|G_{1}\right\|_{L_{t}^{\infty}\left([0,+\infty), L_{x}^{1}\right)} \leq C \epsilon^{2}$

$$
\begin{aligned}
& -\sigma_{3}\left(\dot{\vartheta}-\frac{v \cdot \dot{D}}{2}+\frac{v^{2}}{4}-\omega_{0}\right) f^{\prime}-\mathrm{i}(\dot{D}-v) \cdot \nabla_{x} f^{\prime}-\frac{\dot{v} \cdot x}{2} \sigma_{3} f^{\prime}+\mathrm{i} \dot{f}^{\prime} \\
& =\sigma_{3}\left(-\Delta+\omega_{0}\right) f^{\prime}+G_{1} .
\end{aligned}
$$

Now in (13.18) we substitute the variables of the last coordinate system. In particular $f^{\prime}$ and $f$ are related by a formula such as (8.15):

$$
\begin{aligned}
& f^{\prime}(x)=e^{\mathrm{i} \sigma_{3}\left(-\frac{1}{2} v \cdot(x-\mathbb{A})-\mathrm{i} \widehat{\gamma}\right)} f(x-\mathbb{A})+\mathcal{G}(x), \\
& \widehat{\gamma}=\widetilde{\gamma}+\sum_{\ell=1}^{2 N+1} \gamma_{\ell}, \quad \mathbb{A}=\mathbf{A}+\sum_{\ell=1}^{2 N+1} \mathbf{A}_{\ell},
\end{aligned}
$$

with the $(\widetilde{\gamma}, \mathbf{A})$ of (13.15) and the $\left(\gamma_{\ell}, \mathbf{A}_{\ell}\right)$ of Lemma 13.6. Substituting (13.19) in (13.18) we get after various cancelations, for $\left\|G_{2}\right\|_{L_{t}^{\infty}\left([0,+\infty), L_{x}^{1} \cap H_{x}^{1}\right)} \leq C \epsilon$,

$$
-\sigma_{3}\left(\dot{\vartheta}-\omega_{0}\right) f-\mathrm{i}(\dot{D}+\dot{\mathbb{A}}) \cdot \nabla f+\mathrm{i} \dot{f}=\sigma_{3}\left(-\Delta+\omega_{0}\right) f+G_{2}
$$

We claim that equation (13.20) is equivalent to equation (13.8). Indeed, taking their difference we have

$$
a_{0}(t) \sigma_{3} f+\mathrm{i} a_{j}(t) \partial_{x_{j}} f=\mathbf{G}
$$

with $\mathbf{G}$ (resp. $a_{j}$ ) a continuous functional with values $L^{\infty}\left(\mathbb{R}, L^{1}\left(\mathbb{R}^{3}\right) \cap H_{x}^{1}\right)$ (resp. $L^{\infty}(\mathbb{R})$ ) bounded in the space of solutions we are considering. Then $a_{0}(t) \int f(t, x) d x$ $=\sigma_{3} \int \mathbf{G} d x$. If $a_{0}\left(t_{0}\right) \neq 0$ for a given solution, we can find solutions for which $f_{n}(t, \cdot) \in \mathcal{S}\left(\mathbb{R}^{3}\right), f_{n}\left(t_{0}, \cdot\right) \rightarrow f\left(t_{0}, \cdot\right)$ in $H^{1}\left(\mathbb{R}^{3}\right),\left\|f_{n}\left(t_{0}\right)\right\|_{L^{1}\left(\mathbb{R}^{3}\right)} \nearrow \infty, \mathbf{G}_{n}\left(t_{0}\right) \rightarrow$ $\mathbf{G}\left(t_{0}\right)$ and $a_{0 n}\left(t_{0}\right) \rightarrow a_{0}\left(t_{0}\right)$. This yields a contradiction. So $a_{0}(t) \equiv 0$. By similar reasons $a_{j}(t) \equiv 0$. This implies $\mathbf{G} \equiv 0$. Equivalence of (13.20) and (13.8) yields

$$
\dot{\vartheta}+\frac{1}{2} \frac{d}{d t}(v \cdot \mathbb{A})-\mathrm{i} \dot{\gamma}=\dot{\chi}, \quad \dot{D}_{a}+\dot{\mathbb{A}}_{a}=\partial_{\Pi_{a}(f)} H,
$$

with the first one a consequence of $\dot{\vartheta}-\omega_{0}=\partial_{Q(f)} H$. Using the notation of Lemmas 13.5 13.7, (13.21) yields the following:

$$
\begin{aligned}
& \dot{\theta}=\dot{\vartheta}+\frac{1}{2} \frac{d}{d t}(v \cdot \mathbb{A}), \\
& \dot{D}+\dot{\mathbb{A}}=\dot{X}=\dot{\widehat{D}}+\sum_{\ell=1}^{2 N+1} \dot{\mathbf{A}}_{\ell}=\dot{y}+\dot{\mathbb{A}} \text {, and so } \dot{y}=\dot{D} .
\end{aligned}
$$

Notice that by Lemma 8.4 and inequality (11.8) there exists $\lim _{t \infty} \mathbb{A}(t)$. By Lemma 13.7 we have $\lim _{t \nearrow_{\infty}} v(t)=v_{+}$. This proves the existence of $\vartheta_{0}$ in (13.16), which is then proved.

Lemma 13.9. The functions $(\vartheta, D)$ in Lemma 13.8 satisfy $\dot{D}=v+o(1)$ and $\dot{\vartheta}=\omega+\frac{v^{2}}{4}+o(1)$, with $\lim _{t \rightarrow \infty} o(1)=0$.

Proof. Consider (13.17) with $\varpi=\omega$. Applying to it the linear operator $\left|x_{a} \Phi_{\omega}\right\rangle$ and using the $(z, f)$ of the initial coordinate system (2.14) we get $\left|\dot{D}_{a}-v_{a}\right| \leq$ $C\left(|z|+\|f\|_{L^{2,-S}}\right)$ for arbitrary $S$ and fixed $C$. So the rhs is $o(1)$ and we conclude that $\dot{D}=v+o(1)$. Applying $\left|\sigma_{3} \partial_{\omega} \Phi_{\omega}\right\rangle$ to (13.17) we similarly obtain $\left|\dot{\vartheta}-\frac{v \cdot \dot{D}}{2}+\frac{v^{2}}{4}-\omega\right| \leq$ $C\left(|z|+\|f\|_{L^{2,-S}}\right)$. Using $\dot{D}=v+o(1)$ we conclude $\dot{\vartheta}=\omega+\frac{v^{2}}{4}+o(1)$. 
13.2. Steps (ii) and (iii): The Fermi golden rule. Step (ii) in the proof of Theorem 13.1 consists of introducing the variable

$$
g=f+\sum_{\left|\lambda^{0} \cdot(\mu-\nu)\right|>\omega_{0}} z^{\mu} \bar{z}^{\nu} R_{\mathcal{H}}^{+}\left(\lambda^{0} \cdot(\mu-\nu)\right) G_{\mu \nu}^{0} .
$$

Substituting the new variable $g$ in (13.8), the first line on the rhs of (13.8) cancels out. The following result has been proved in a variety of places in the absence of translation; see for example $[\mathrm{BuC}]$. Thanks to Section 3.3 of $[\mathrm{Be}$, essentially the same proof holds here. We skip the proof.

Lemma 13.10. For $\epsilon$ in Theorem 9.1 sufficiently small, for $C_{0}=C_{0}(\mathcal{H})$ a fixed constant, we have $\|g\|_{L_{t}^{2} L_{x}^{2,-S}} \leq C_{0} \epsilon+O\left(\epsilon^{2}\right)$ for a fixed $S>1$.

We have arrived at step (iii) of the proof of Theorem 13.1 the Fermi golden rule.

Proposition 13.11. There is a new set of variables $\zeta=z+O\left(z^{2}\right)$ such that for a fixed $C$ we have

$$
\|\zeta-z\|_{L_{t}^{2}} \leq C C_{2} \epsilon^{2}, \quad\|\zeta-z\|_{L_{t}^{\infty}} \leq C \epsilon^{3}
$$

and we have

$$
\begin{aligned}
& \partial_{t} \sum_{j=1}^{m} \lambda_{j}^{0}\left|\zeta_{j}\right|^{2}=2 \sum_{j=1}^{m} \lambda_{j}^{0} \operatorname{Im}\left(\mathcal{D}_{j} \bar{\zeta}_{j}\right) \\
& -2 \sum_{\substack{\lambda=\lambda^{0} \cdot \alpha=\lambda^{0} \cdot \nu>\omega_{0} \\
\lambda \cdot \alpha-\lambda_{k}<\omega_{0} \forall k \\
\lambda \cdot \nu-\lambda_{k}<\omega_{0} \forall k \text { s.t. } \alpha_{k} \neq 0 \\
\text { s.t. } \nu_{k} \neq 0}} \lambda^{0} \cdot \nu \operatorname{Im}\left(\zeta^{\alpha} \bar{\zeta}^{\nu}\left\langle R_{\alpha 0}^{+} G_{\alpha 0}^{0} \mid \sigma_{1} \sigma_{3} G_{0 \nu}^{0}\right\rangle\right),
\end{aligned}
$$

where $\sum_{j}\left\|\mathcal{D}_{j} \bar{\zeta}_{j}\right\|_{L^{1}[0, T]} \leq\left(1+C_{2}\right) c_{0} \epsilon^{2}$ for a fixed constant $c_{0}$.

Proof. See [Cu1] and [Cu4].

For the sum in the second line of (13.25) we get finite sums

$$
\begin{aligned}
& 2 \sum_{\Lambda>\omega_{0}} \Lambda \operatorname{Im}\left\langle R_{\mathcal{H}}^{+}(\Lambda) \sum_{\lambda^{0} \cdot \alpha=\Lambda} \zeta^{\alpha} G_{\alpha 0}^{0} \mid \sigma_{1} \sigma_{3} \sum_{\lambda^{0} \cdot \nu=\Lambda} \bar{\zeta}^{\nu} G_{0 \nu}^{0}\right\rangle \\
& =2 \sum_{\Lambda>\omega_{0}} \Lambda \operatorname{Im}\left\langle R_{\mathcal{H}}^{+}(\Lambda) \sum_{\lambda^{0} \cdot \alpha=\Lambda} \zeta^{\alpha} G_{\alpha 0}^{0} \mid \sigma_{3} \overline{\sum_{\lambda^{0} \cdot \alpha=\Lambda} \zeta^{\alpha} G_{\alpha 0}^{0}}\right\rangle,
\end{aligned}
$$

where we have used $G_{\mu \nu}^{0}=-\sigma_{1} \bar{G}^{0}{ }_{\nu \mu}$. Notice that the existence of $R_{\mathcal{H}}^{+}(\Lambda)$ for $\Lambda \in \sigma_{e}(\mathcal{H})$ is proved in [CPV].

We have:

Lemma 13.12 (Semipositivity). We have rhs $(13.26) \geq 0$.

Proof. See Cu1.

Now we will assume the following hypothesis.

(H10) We assume that for some fixed constants for any vector $\zeta \in \mathbb{C}^{n}$ we have

$$
\sum_{\substack{(\alpha, \nu) \text { as } \\ \text { in (13.25) }}} \lambda^{0} \cdot \nu \operatorname{Im}\left(\zeta^{\alpha} \bar{\zeta}^{\nu}\left\langle R_{\alpha 0}^{+} G_{\alpha 0}^{0} \mid \sigma_{1} \sigma_{3} G_{0 \nu}^{0}\right\rangle\right) \approx \sum_{\substack{\lambda^{0} \cdot \alpha>\omega_{0} \\ \lambda^{0} \cdot \alpha-\lambda_{k}^{0}<\omega_{0} \\ \forall k \text { s.t. } \alpha k \neq 0}}\left|\zeta^{\alpha}\right|^{2} .
$$


By (H10) we have

$$
2 \sum_{j} \lambda_{j}^{0} \operatorname{Im}\left(\mathcal{D}_{j} \bar{\zeta}_{j}\right) \gtrsim \partial_{t} \sum_{j} \lambda_{j}^{0}\left|\zeta_{j}\right|^{2}+\sum_{\alpha \text { as in }(\mathrm{H} 10)}\left|\zeta^{\alpha}\right|^{2} .
$$

Then, for $t \in[0, T]$, by the last line in Proposition 13.11 we have

$$
\sum_{j} \lambda_{j}^{0}\left|\zeta_{j}(t)\right|^{2}+\sum_{\alpha \text { as in (H10) }}\left\|\zeta^{\alpha}\right\|_{L^{2}(0, t)}^{2} \lesssim \epsilon^{2}+C_{2} \epsilon^{2} .
$$

By (13.24) this implies $\left\|z^{\alpha}\right\|_{L^{2}(0, t)}^{2} \lesssim \epsilon^{2}+C_{2} \epsilon^{2}$ for all the above multiindexes. So, from $\left\|z^{\alpha}\right\|_{L^{2}(0, t)}^{2} \leq C_{2}^{2} \epsilon^{2}$ we conclude $\left\|z^{\alpha}\right\|_{L^{2}(0, t)}^{2} \leq \kappa C_{2} \epsilon^{2}$ for a fixed $\kappa$, which is an improvement if $C_{2}$ is sufficiently large. So if we take $C_{1}>2 K_{1}\left(C_{2}\right)$ and $C_{1}-C_{2}$ sufficiently large, in particular so that $\sqrt{\kappa C_{2}}<C_{2} / 2$, we conclude as desired that (13.4) and (13.5) imply the same estimate but with $C_{1}, C_{2}$ replaced by $C_{1} / 2, C_{2} / 2$. This yields Theorem 13.1

Remark 13.13. Suppose for simplicity that $\lambda_{1}(\omega)=\ldots=\lambda_{m}(\omega)=: \lambda(\omega)$ and let us see the meaning of (H10). We have $G_{\alpha 0}^{0} \in \mathcal{S}\left(\mathbb{R}^{3}, \mathbb{C}^{2}\right)$ for all $\alpha$. For $W(\omega)=$ $\lim _{t \rightarrow+\infty} e^{-\mathrm{i} t \mathcal{H}_{\omega}} e^{\mathrm{i} t \sigma_{3}(-\Delta+\omega)}$, there exist $F_{\alpha} \in W^{k, p}\left(\mathbb{R}^{3}, \mathbb{C}^{2}\right)$ for all $k \in \mathbb{R}$ and $p \geq 1$ with $G_{\alpha 0}^{0}=W\left(\omega_{0}\right) F_{\alpha}$, [Cu6]. Let ${ }^{t} F_{\alpha}=\left(F_{\alpha}^{(1)}, F_{\alpha}^{(2)}\right)$. Then the lhs of (H10) can be expressed as

$$
\int_{|\xi|=\sqrt{(N+1) \lambda_{0}-\omega_{0}}}\left|\sum_{|\alpha|=N+1} \zeta^{\alpha} \widehat{F}_{\alpha}^{(1)}(\xi)\right|^{2} d S(\xi),
$$

where we are taking the standard Fourier transform and $d S(\xi)$ is the standard measure on a sphere. (13.27) is equivalent to the linear independence of the finite family of functions $\left\{\widehat{F}_{\alpha}^{(1)}\right\}_{\alpha}$ on the sphere of radius $\sqrt{(N+1) \lambda_{0}-\omega_{0}}$. This independence is in general expected to be true. This point is discussed in [GW] for a special situation involving small ground states and $N=1$.

\section{ACKNOWLEDGMENTS}

The author wishes to thank G. Tondo for discussions about the reduction of variables and for pointing out reference $\mathrm{O}$.

\section{REFERENCES}

[BC] Dario Bambusi and Scipio Cuccagna, On dispersion of small energy solutions to the nonlinear Klein Gordon equation with a potential, Amer. J. Math. 133 (2011), no. 5, 14211468, DOI 10.1353/ajm.2011.0034. MR.2843104

[Be] M. Beceanu, A Critical Centre-Stable Manifold for Schrödinger's Equation in $\mathbb{R}^{3}$, Comm. Pure Appl. Math. 65 (2012), no. 4, 431-507. MR2877342

[BuC] Nabile Boussaid and Scipio Cuccagna, On stability of standing waves of nonlinear Dirac equations, Comm. Partial Differential Equations 37 (2012), no. 6, 1001-1056, DOI 10.1080/03605302.2012.665973. MR2924465

[BP1] V. S. Buslaev and G. S. Perel'man, Scattering for the nonlinear Schrödinger equation: states that are close to a soliton (Russian, with Russian summary), Algebra i Analiz 4 (1992), no. 6, 63-102; English transl., St. Petersburg Math. J. 4 (1993), no. 6, 1111-1142. MR1199635 (94b:35256)

[BP2] V. S. Buslaev and G. S. Perel'man, On the stability of solitary waves for nonlinear Schrödinger equations, Nonlinear evolution equations, Amer. Math. Soc. Transl. Ser. 2, vol. 164, Amer. Math. Soc., Providence, RI, 1995, pp. 75-98. MR.1334139 (96e:35157) 
[BS] Vladimir S. Buslaev and Catherine Sulem, On asymptotic stability of solitary waves for nonlinear Schrödinger equations (English, with English and French summaries), Ann. Inst. H. Poincaré Anal. Non Linéaire 20 (2003), no. 3, 419-475, DOI 10.1016/S02941449(02)00018-5. MR.1972870 (2004d:35229)

[CL] T. Cazenave and P.-L. Lions, Orbital stability of standing waves for some nonlinear Schrödinger equations, Comm. Math. Phys. 85 (1982), no. 4, 549-561. MR677997 (84i:81015)

[Cu1] Scipio Cuccagna, The Hamiltonian structure of the nonlinear Schrödinger equation and the asymptotic stability of its ground states, Comm. Math. Phys. 305 (2011), no. 2, 279331, DOI 10.1007/s00220-011-1265-2. MR2805462 (2012d:37176)

[Cu2] Scipio Cuccagna, On asymptotic stability of ground states of NLS, Rev. Math. Phys. 15 (2003), no. 8, 877-903, DOI 10.1142/S0129055X03001849. MR2027616 (2004k:35348)

[Cu3] Scipio Cuccagna, On instability of excited states of the nonlinear Schrödinger equation, Phys. D 238 (2009), no. 1, 38-54, DOI 10.1016/j.physd.2008.08.010. MR2571965 (2010m:35482)

[Cu4] Scipio Cuccagna, On scattering of small energy solutions of non-autonomous Hamiltonian nonlinear Schrödinger equations, J. Differential Equations 250 (2011), no. 5, 2347-2371, DOI 10.1016/j.jde.2010.11.014. MR2756067(2012e:35237)

[Cu5] Scipio Cuccagna, On asymptotic stability in energy space of ground states of NLS in 1D, J. Differential Equations 245 (2008), no. 3, 653-691, DOI 10.1016/j.jde.2008.02.042. MR2422523 (2009c:35435)

[Cu6] Scipio Cuccagna, Stabilization of solutions to nonlinear Schrödinger equations, Comm. Pure Appl. Math. 54 (2001), no. 9, 1110-1145, DOI 10.1002/cpa.1018. MR1835384 (2002g:35193)

[CM] Scipio Cuccagna and Tetsu Mizumachi, On asymptotic stability in energy space of ground states for nonlinear Schrödinger equations, Comm. Math. Phys. 284 (2008), no. 1, 51-77, DOI 10.1007/s00220-008-0605-3. MR.2443298(2009k:35294)

[CPV] Scipio Cuccagna, Dmitry Pelinovsky, and Vitali Vougalter, Spectra of positive and negative energies in the linearized NLS problem, Comm. Pure Appl. Math. 58 (2005), no. 1, 1-29, DOI 10.1002/cpa.20050. MR2094265 (2005k:35374)

[GS] Zhou Gang and I. M. Sigal, Relaxation of solitons in nonlinear Schrödinger equations with potential, Adv. Math. 216 (2007), no. 2, 443-490, DOI 10.1016/j.aim.2007.04.018. MR.2351368 (2008k:35441)

[GW] Zhou Gang and Michael I. Weinstein, Dynamics of nonlinear Schrödinger/GrossPitaevskii equations: mass transfer in systems with solitons and degenerate neutral modes, Anal. PDE 1 (2008), no. 3, 267-322, DOI 10.2140/apde.2008.1.267. MR.2490293 (2010j:35499)

[GSS1] Manoussos Grillakis, Jalal Shatah, and Walter Strauss, Stability theory of solitary waves in the presence of symmetry. I, J. Funct. Anal. 74 (1987), no. 1, 160-197, DOI 10.1016/00221236(87)90044-9. MR901236 (88g:35169)

[GSS2] Manoussos Grillakis, Jalal Shatah, and Walter Strauss, Stability theory of solitary waves in the presence of symmetry. II, J. Funct. Anal. 94 (1990), no. 2, 308-348, DOI 10.1016/00221236(90)90016-E. MR.1081647 (92a:35135)

[KW] E. Kirr and M. I. Weinstein, Diffusion of power in randomly perturbed Hamiltonian partial differential equations, Comm. Math. Phys. 255 (2005), no. 2, 293-328, DOI 10.1007/s00220-004-1273-6. MR 2129948 (2006e:35273)

[MS] H. P. McKean and J. Shatah, The nonlinear Schrödinger equation and the nonlinear heat equation reduction to linear form, Comm. Pure Appl. Math. 44 (1991), no. 8-9, 1067-1080, DOI 10.1002/cpa.3160440817. MR.1127050 (93b:35126)

[N] Edward Nelson, Topics in dynamics. I: Flows, Mathematical Notes, Princeton University Press, Princeton, N.J., 1969. MR.0282379 (43 \#8091)

[O] Peter J. Olver, Applications of Lie groups to differential equations, 2nd ed., Graduate Texts in Mathematics, vol. 107, Springer-Verlag, New York, 1993. MR.1240056 (94g:58260)

[P] Galina Perelman, Asymptotic stability of multi-soliton solutions for nonlinear Schrödinger equations, Comm. Partial Differential Equations 29 (2004), no. 7-8, 1051-1095, DOI 10.1081/PDE-200033754. MR2097576 (2005g:35277)

[RSS] I. Rodnianski, W. Schlag, A. Soffer, Asymptotic stability of N-soliton states of NLS, (2003), arXiv:math/0309114v1. 
[S] Jalal Shatah, Stable standing waves of nonlinear Klein-Gordon equations, Comm. Math. Phys. 91 (1983), no. 3, 313-327. MR723756 (84m:35111)

[SW1] A. Soffer and M. I. Weinstein, Multichannel nonlinear scattering for nonintegrable equations, Comm. Math. Phys. 133 (1990), no. 1, 119-146. MR1071238(91h:35303)

[SW2] A. Soffer and M. I. Weinstein, Multichannel nonlinear scattering for nonintegrable equations. II. The case of anisotropic potentials and data, J. Differential Equations 98 (1992), no. 2, 376-390, DOI 10.1016/0022-0396(92)90098-8. MR.1170476 (93i:35137)

[SW3] A. Soffer and M. I. Weinstein, Resonances, radiation damping and instability in Hamiltonian nonlinear wave equations, Invent. Math. 136 (1999), no. 1, 9-74, DOI 10.1007/s002220050303. MR.1681113(2000k:37119)

[Si] I. M. Sigal, Nonlinear wave and Schrödinger equations. I. Instability of periodic and quasiperiodic solutions, Comm. Math. Phys. 153 (1993), no. 2, 297-320. MR1218303 (94d:35012)

[Ta] M. Taylor, Partial differential equations, Appl. Math. Sci. vol. 117, Springer-Verlag, New York, 1996.

[Ts] Tai-Peng Tsai, Asymptotic dynamics of nonlinear Schrödinger equations with many bound states, J. Differential Equations 192 (2003), no. 1, 225-282, DOI 10.1016/S00220396(03)00041-X. MR1987092 (2004h:35212)

[TY1] Tai-Peng Tsai and Horng-Tzer Yau, Asymptotic dynamics of nonlinear Schrödinger equations: resonance-dominated and dispersion-dominated solutions, Comm. Pure Appl. Math. 55 (2002), no. 2, 153-216, DOI 10.1002/cpa.3012. MR1865414 (2002i:35182)

[TY2] Tai-Peng Tsai and Horng-Tzer Yau, Relaxation of excited states in nonlinear Schrödinger equations, Int. Math. Res. Not. 31 (2002), 1629-1673, DOI 10.1155/S1073792802201063. MR1916427 (2004i:35292)

[TY3] Tai-Peng Tsai and Horng-Tzer Yau, Classification of asymptotic profiles for nonlinear Schrödinger equations with small initial data, Adv. Theor. Math. Phys. 6 (2002), no. 1, 107-139. MR:1992875 (2004m:35254)

[W1] Michael I. Weinstein, Lyapunov stability of ground states of nonlinear dispersive evolution equations, Comm. Pure Appl. Math. 39 (1986), no. 1, 51-67, DOI 10.1002/cpa.3160390103. MR820338 (87f:35023)

[W2] Michael I. Weinstein, Modulational stability of ground states of nonlinear Schrödinger equations, SIAM J. Math. Anal. 16 (1985), no. 3, 472-491, DOI 10.1137/0516034. MR783974 (86i:35130)

Department of Mathematics and Computer Sciences, University of Trieste, via VaLERIO 12/1 TRIESTE, 34127 ItAly

E-mail address: scuccagna@units.it 